\title{
Testable Design and Testing of Microsystems
}

\author{
Hans G. Kerkhoff, The Netherlands
}

http://www.ewh.ieee.org/tc/sensors/sensors2005/

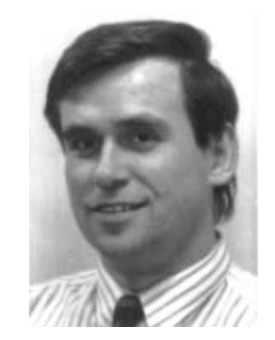

\section{Abstract:}

The market of microsystems, incorporating microelectronics with e.g. MEMS-based sensors and actuators increases rapidly. The often non-electrical sensors and actuators are notoriously difficult and/or expensive to test. As the entire micro system has to meet certain quality criteria for specific applications, new techniques are being developed.

This tutorial will show current test practices of several basic categories of microsystems, and also methods to reduce or circumvent test problems. In these approaches, good knowledge on defects in the systems is required, and design modifications or extensions are shown to ease the testing process.

\section{Biography:}

Hans G. Kerkhoff received his M.Sc. degree in Telecommunication with honours at the Technical University of Delft in 1977 and the Delft Hogeschool Award for his M.Sc. thesis. In the same year he became staff member of the chair IC-Technology \& Electronics at the Faculty of Electrical Engineering, University of Twente in Enschede. He obtained a Ph.D. in Technical Science (micro-electronics) at the University of Twente in 1984. In the same year he was appointed associate professor in Testable Design and Testing at the Faculty of Electrical Engineering at the University of Twente. He advised $15 \mathrm{Ph} . D$. students in this research area and has (co-) authored over 200 publications.

In 1991, he became head of the research group "Testable Design and Test of Microsystems" of the MESA+ Research Institute and headed the MESA Test Centre (MTC). In 1992 he spent his sabbatical year at the test company Advantest in San Jose (CA), USA. From 1995 up to1999, he worked in addition part-time at the Philips Research Laboratories in the VLSI Design and Test Group at Eindhoven. 


\title{
Testable Design for
}

\section{MEMS Sensors}

Hans G. Kerkhoff

\author{
University of Twente
}

Enschede, the Netherlands 


\section{Contents}

Introduction

- MEMS in microsystems applications

- The MEMS market, and the cost of testing

- Testing MEMS

- Functional and structural approaches \& examples

- Domain-specific handlers and ATE

DfT for MEMS

- Domain input and output interfaces \& examples

BIST in MEMS

- Several approaches \& examples

Conclusions 


\section{MEMS: Many Applications}

- Transport

- Safety-critical car microsystems (e.g. airbag)

e) Industrial \& environmental

- Control systems (e.g. pressure, flow)

- Seismic, pH, images etc.

c) Consumer

- Telecommunication systems (e.g. microphones)

- Entertainment (CD/DVD players/recorders)

- Computer peripherals (e.g. discs, inkjet printers)

- Healthcare

- Microfluidic systems (e.g. drug synthesis) 


\section{Test Degree Application Dependent}

Consumer products

Environmental

- Industrial products

- Transport (safety-critical)

Health,

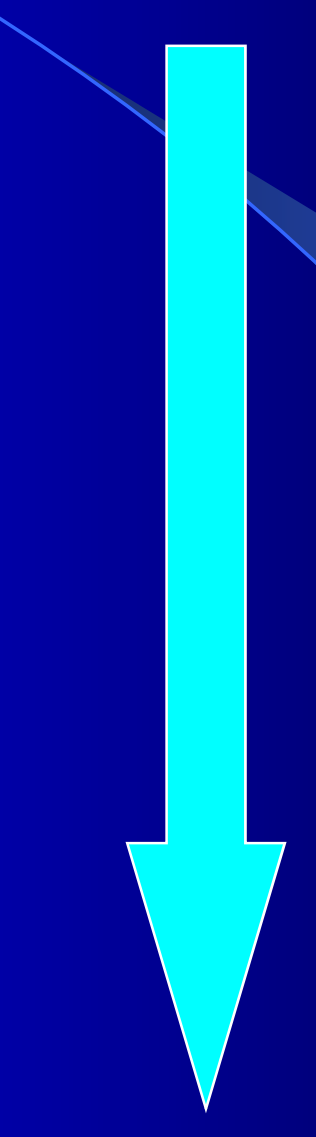
Higher test budget 


\section{Some established ........}

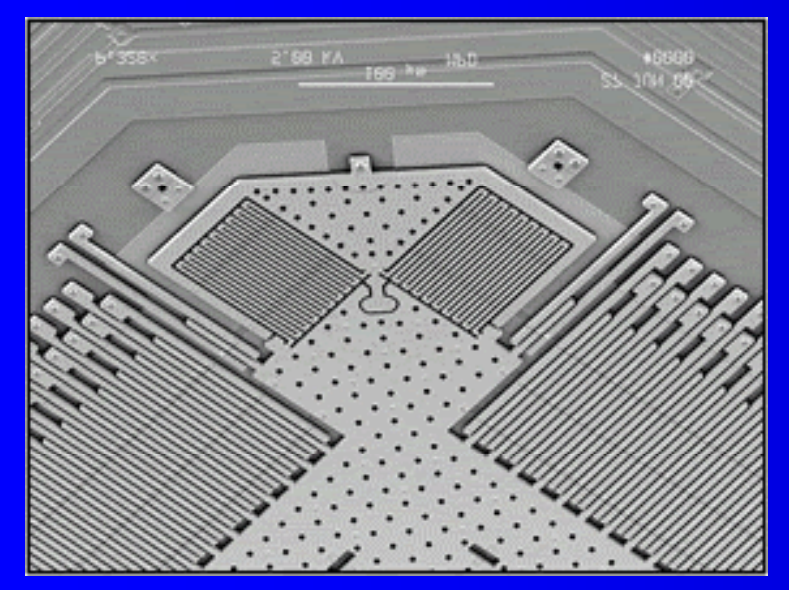

airbag

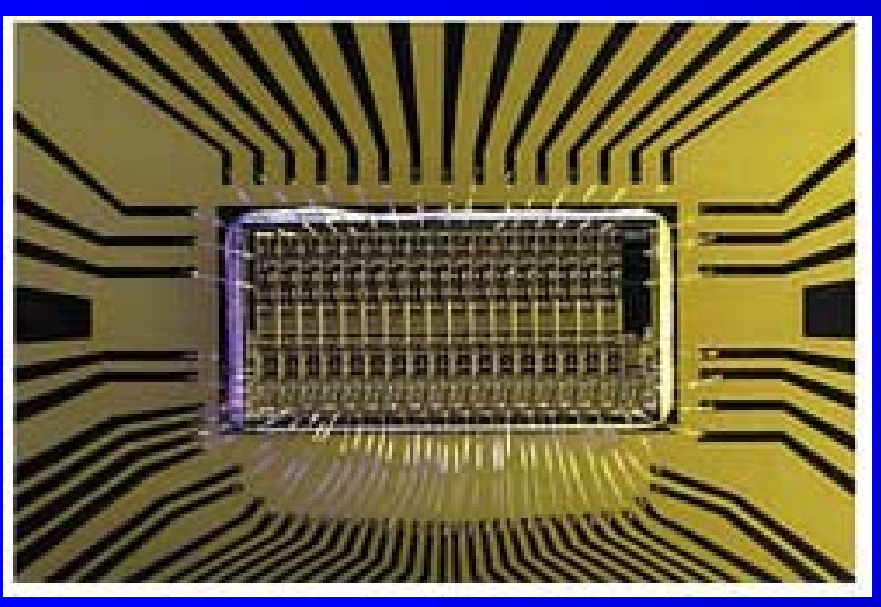

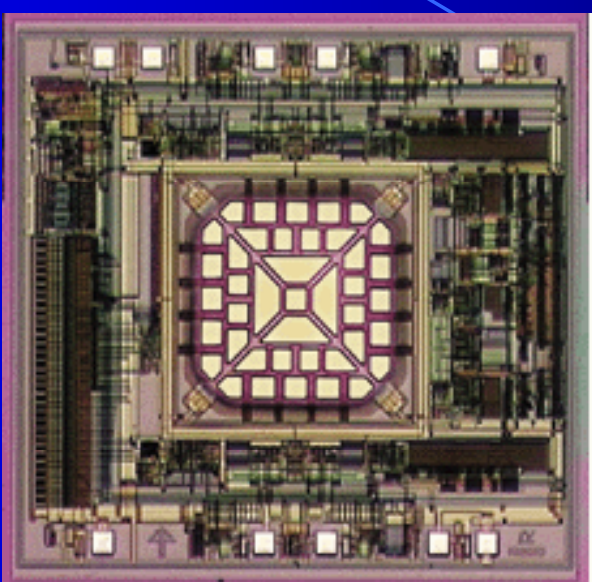

accelerator

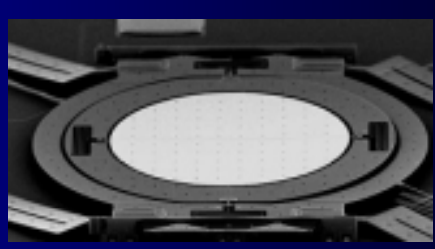

Optical mirror

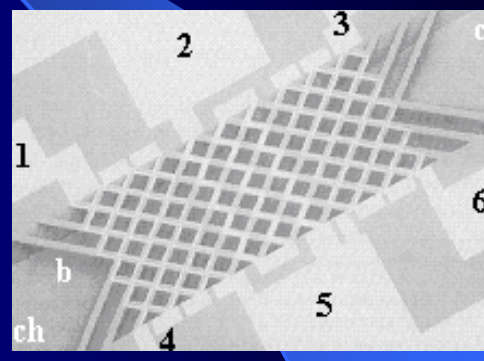

Flow sensing

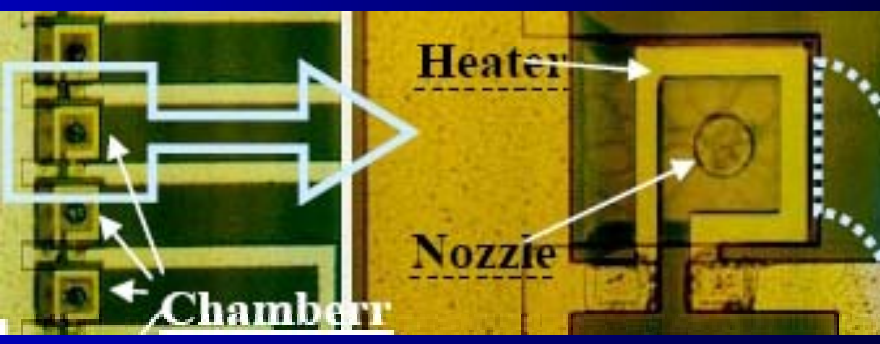

Inkjet printer 


\section{Others promising \& emerging ....}
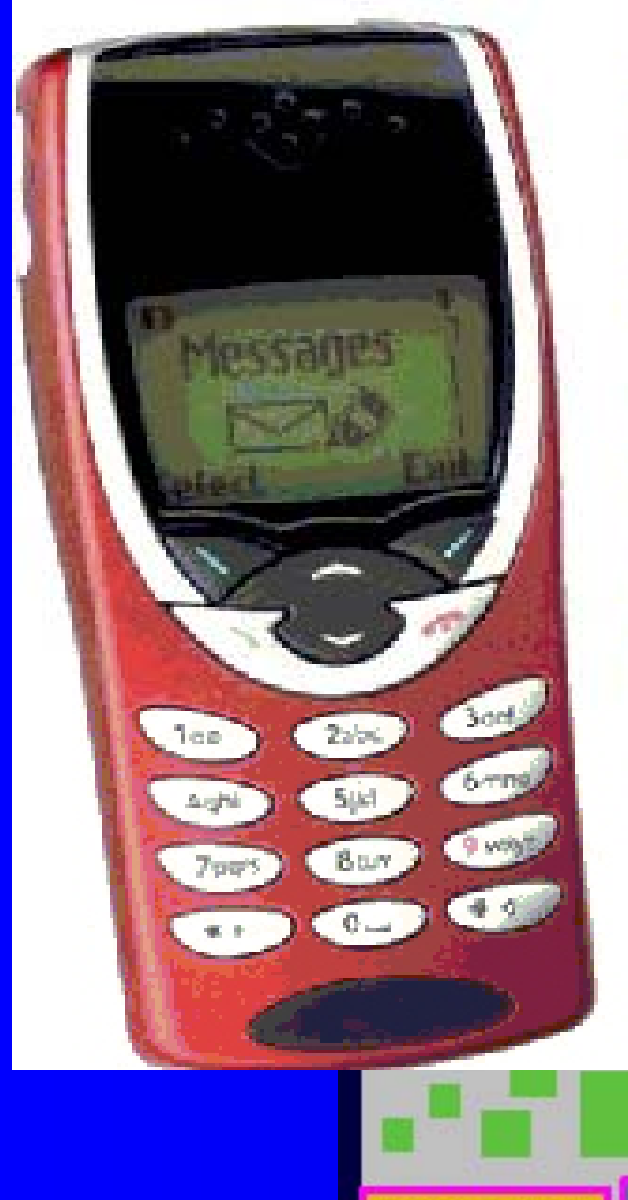

BASE BAND PROCESSOR

$\square \square$
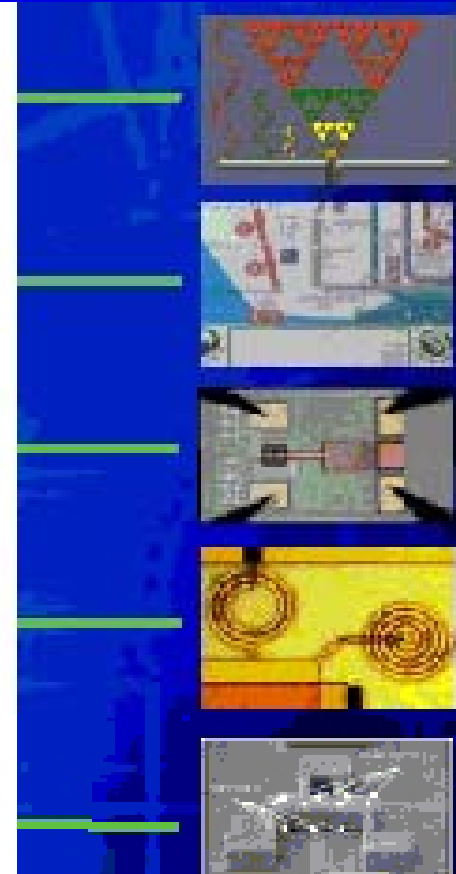

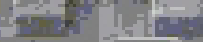

Antennas

Color bi-stable display

Micro-switches

Tunable capacitors and inductors

Tunable filters

Directional microphone

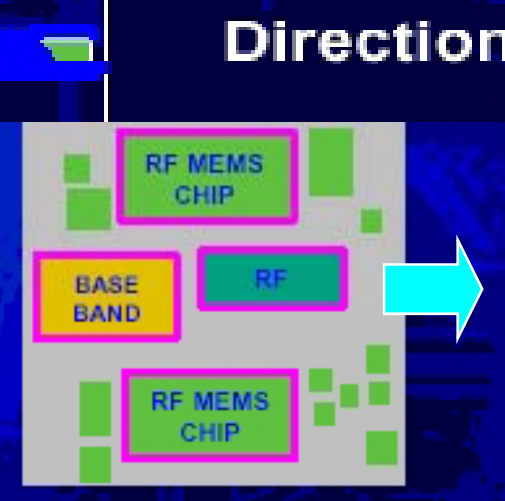

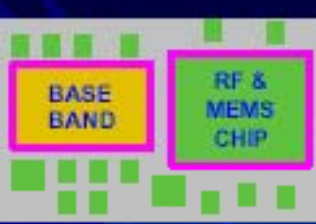

(courtesy INTEL ) 


\section{High-Q Oscillator Testing}

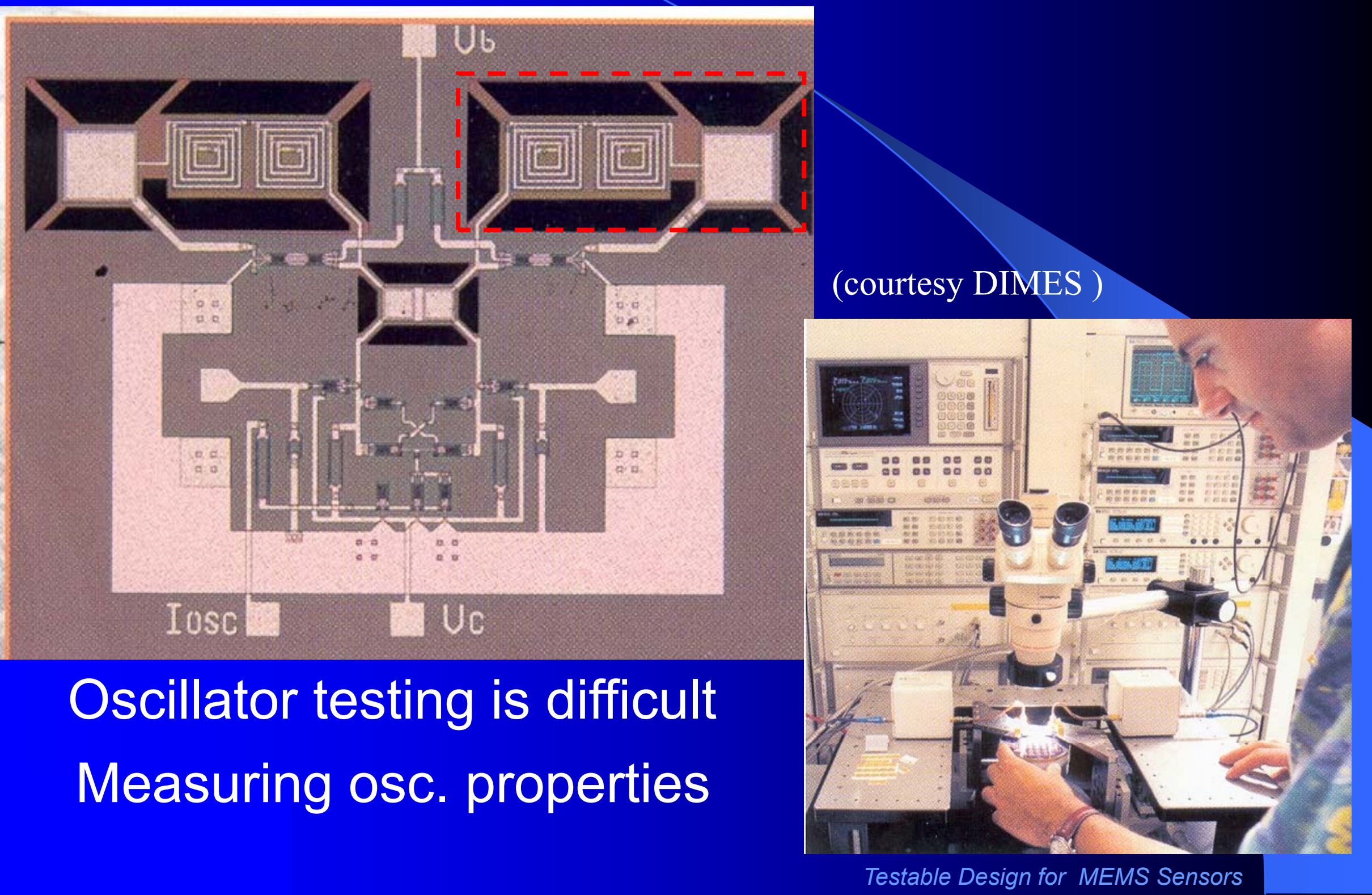




\section{Promising: MOEMS for Interconnect}

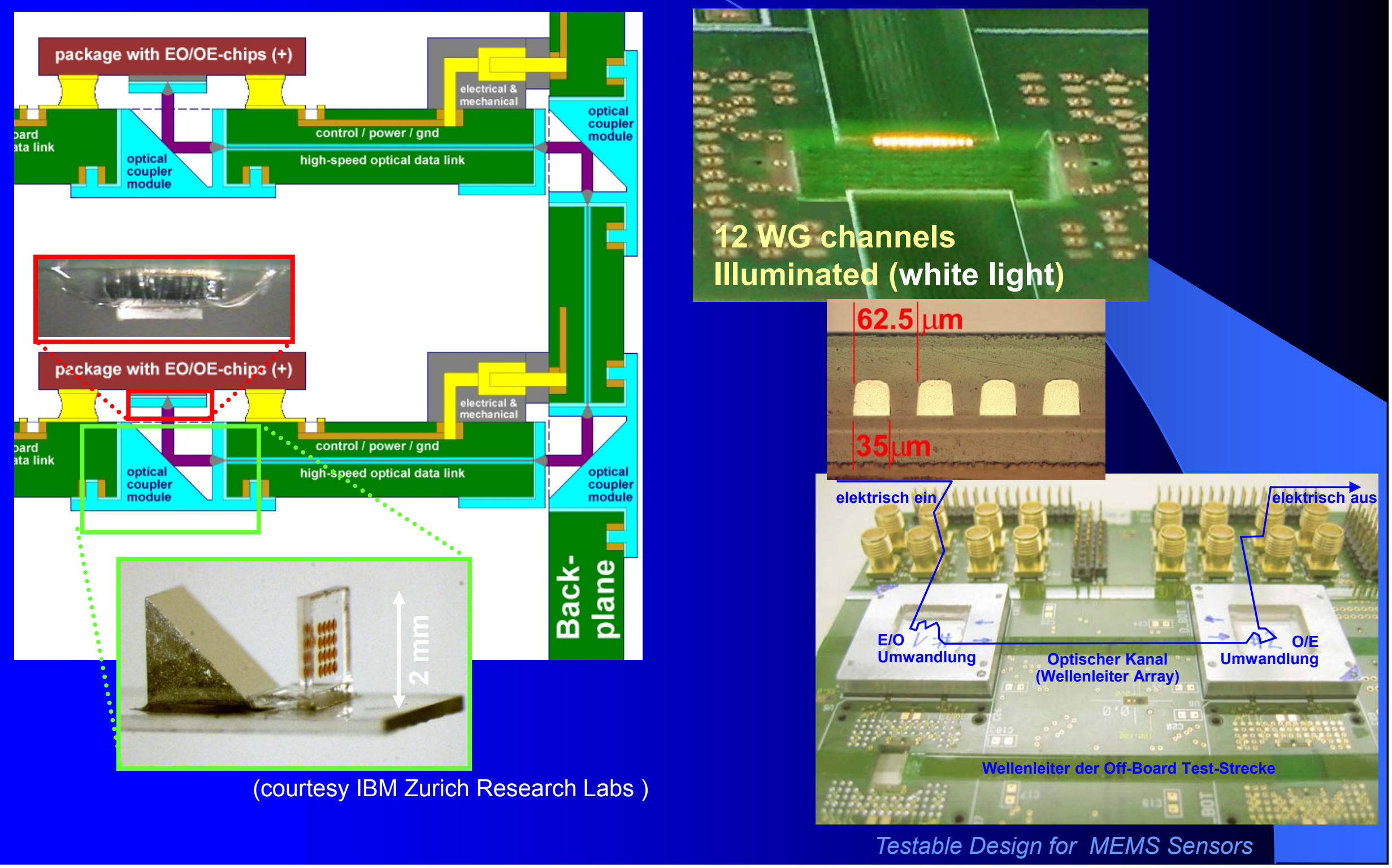




\section{The MEMS Market (1)}

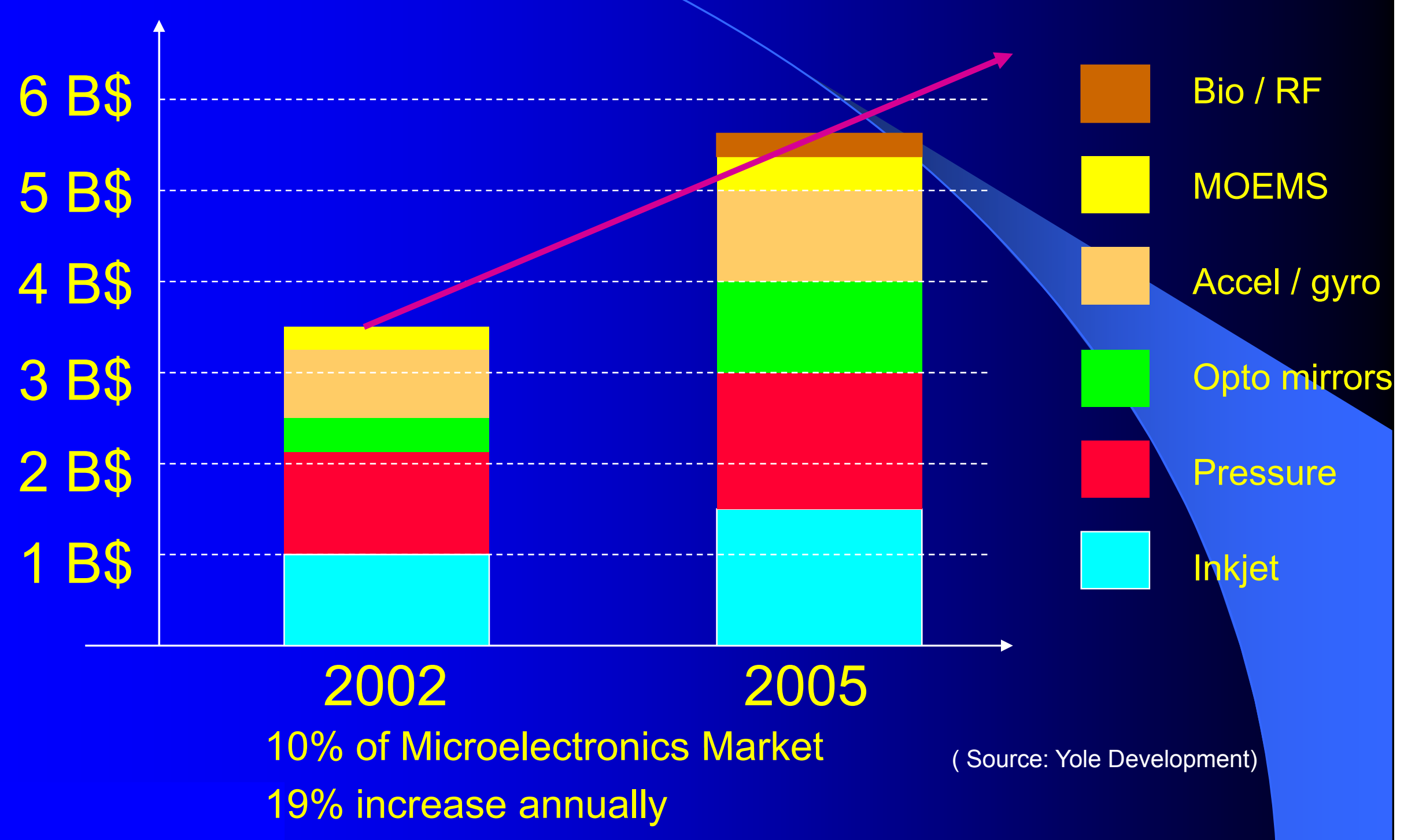




\section{The MEMS Market (2)}

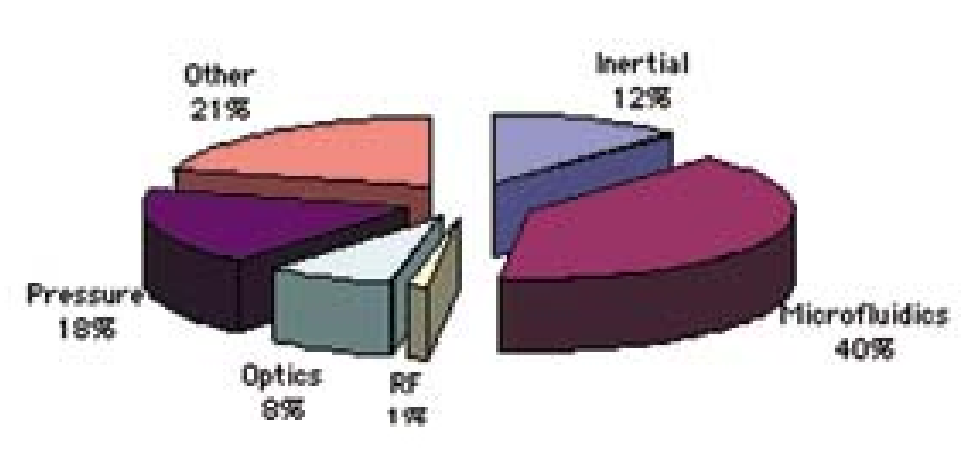

* Conventional

- Inertial (Accelerometers)

- Pressure \& flow

Emerging

- RF MEMS

- Optical MEMS (MOEMS)

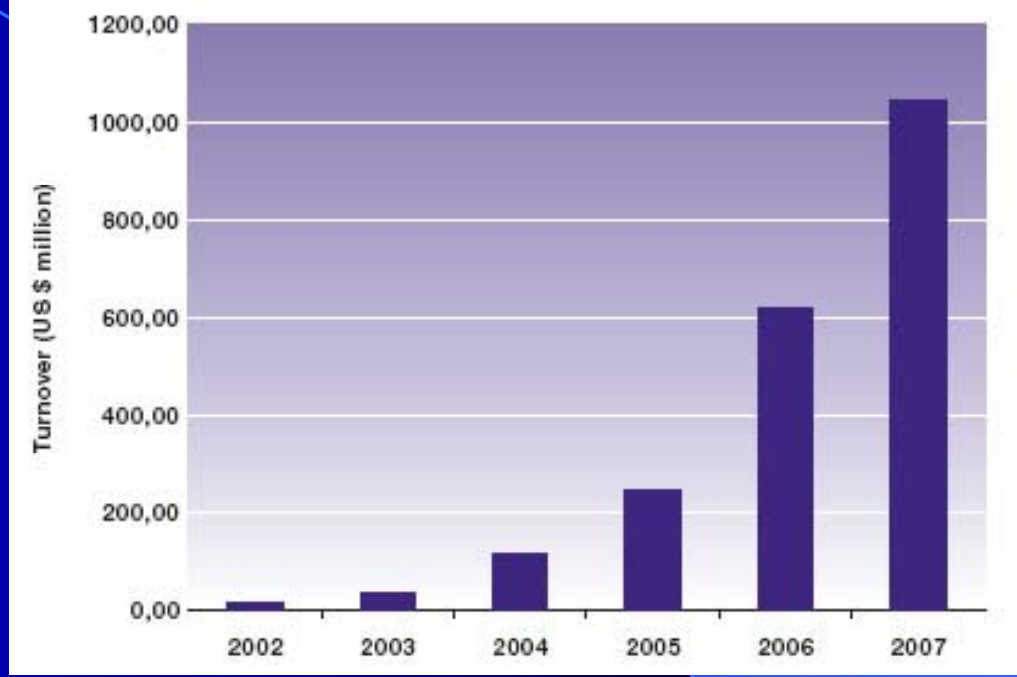

Optical MEMS 2003-2008 Market (Yole source)

- Marlat wil grow from 5756 millon In 2001 to 53098 million in 2006

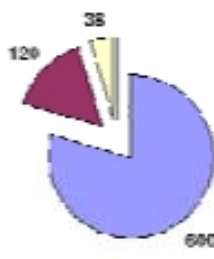

CATR $-33 \%$

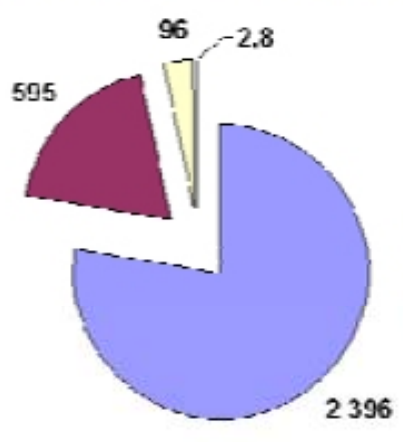

mI:

wiR msga cancor

」जpsctuonesr

Itsure wate redol 


\section{Cost Division in MEMS}

Testing and related packaging can reach $70 \%$ of total costs

Division in costs in pressure MEMS

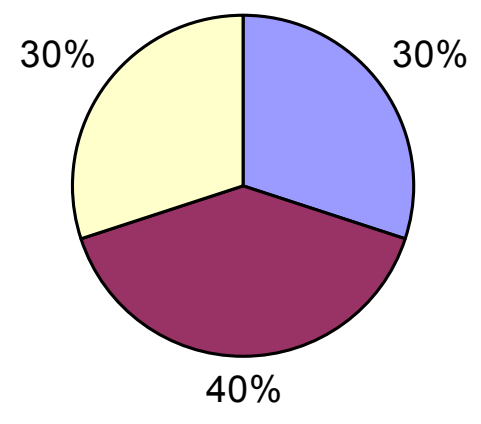

$\square$ Design \&

implement

$\square$ Packaging

$\square$ Testing

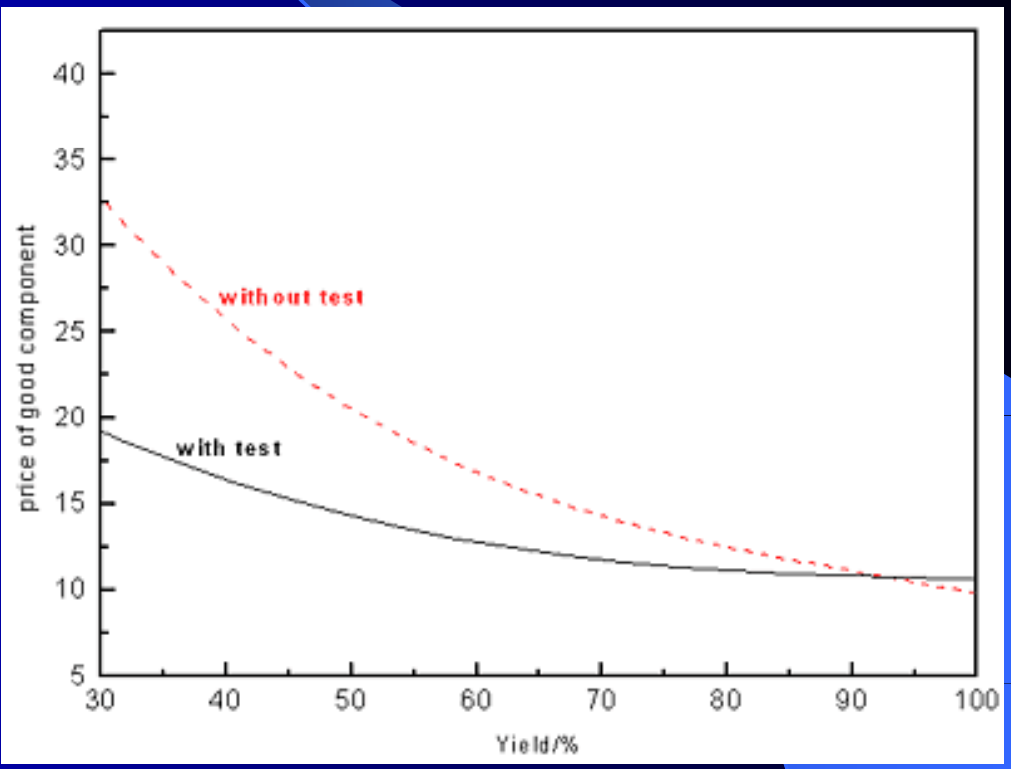

- Studies show that doing electrical wafer tests pays off due to expensive packaging 


\section{MEMS Processing}

Q Substrate

- Silicon

- Other (e.g. glass)

- Bulk micromaching

- Substrate is part of the MEMS operation

- Surface micromaching

- Substrate is carrier of the MEMS structure

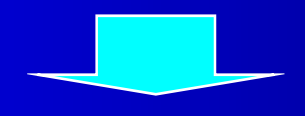

D Different technologies, complete different defects

- besides electrical also other (e.g. mechanical) issues 


\section{Very Many Different Defects ...}

pinholes, tox change

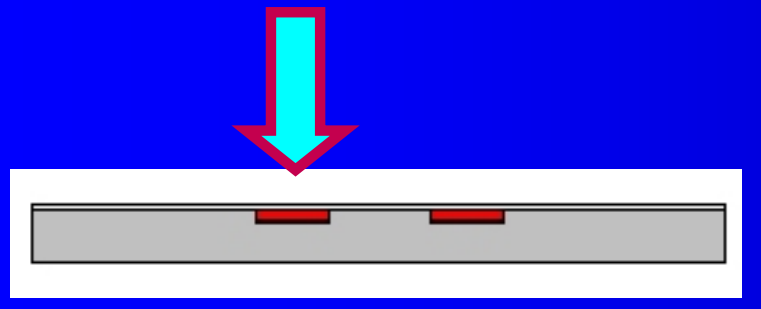

fluidics

- high-R vias,

- Vg variation

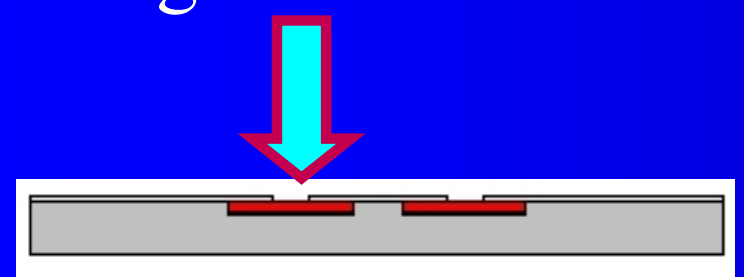

thermal bonding

RF stress \& stiction 


\section{MEMS Microsystem Testing Issues}

- Lack of defect origins \& faultmodels because specific, (relative unmatured) processes

- stiction, cracks, voids, etc. (surface, bulk micromachining, LIGA)

- Multi-domain design, fault simulation \& test

- VHDL-AMS, domain interface circuits

* Heavily influenced by packaging \& environment

- stress, crosstalk \& temperature, humidity, pressure

- No standards \& no knowledge spread by companies

- different customized packages, proprietary solutions 


\section{MEMS, Test and Packaging}

- Microelectronics: protect chip from outside influences

MEMS sensors / actuators: interact with outside

MEMS functional operation critical to package design

Device calibration and test closely linked to package

MEMS packaging often more expensive then device

Difficult to test, except electrical, prior to packaging 


\section{Packaging Interaction Modelling}

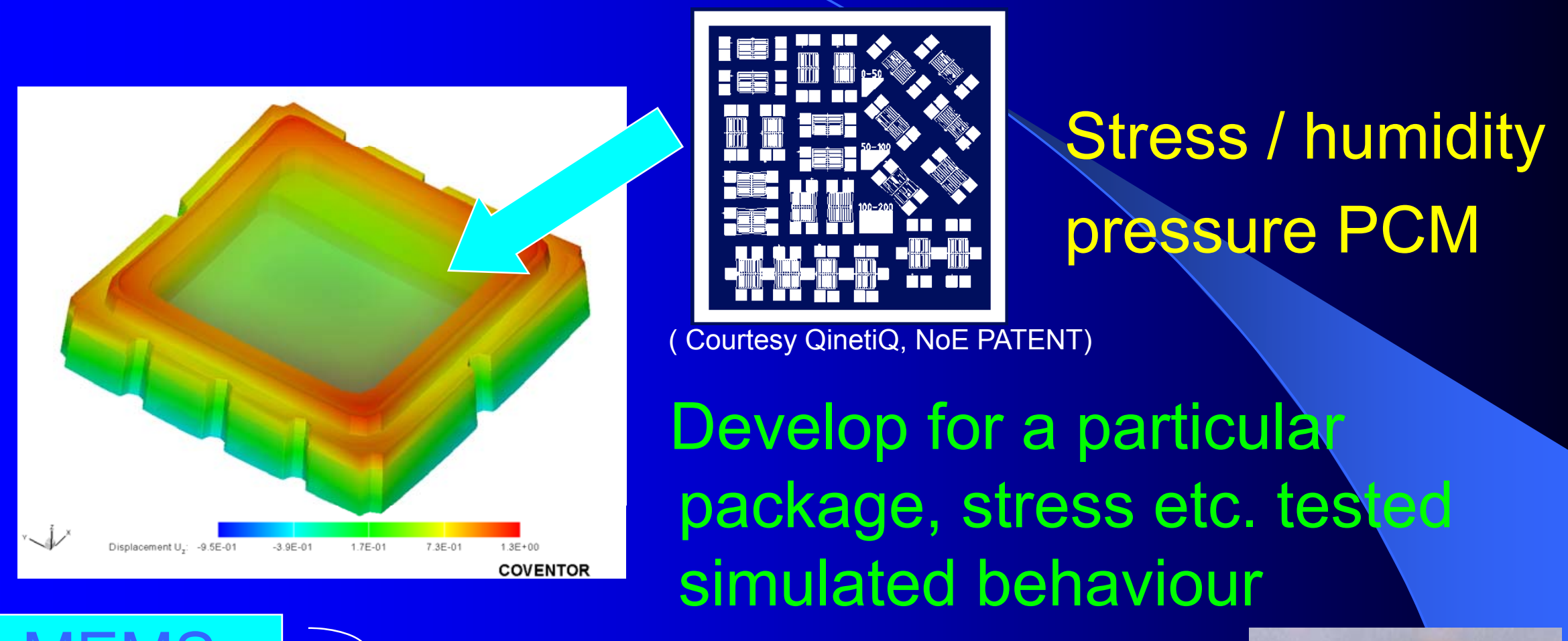

\section{MEMS \\ model}

Package model
Interaction simulation
Real

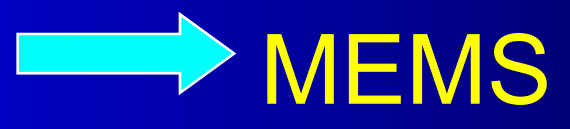
behaviour

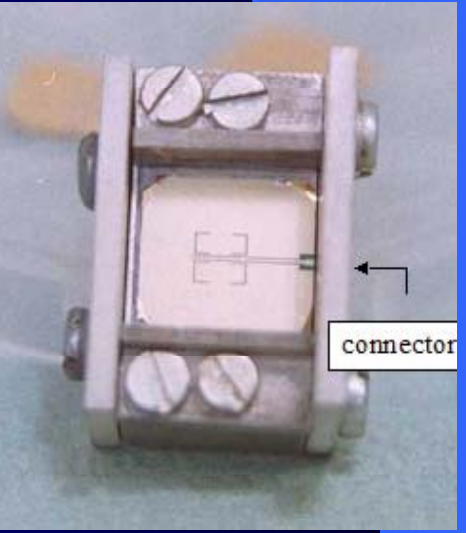




\section{MEMS Design, Modelling \& Simulation}

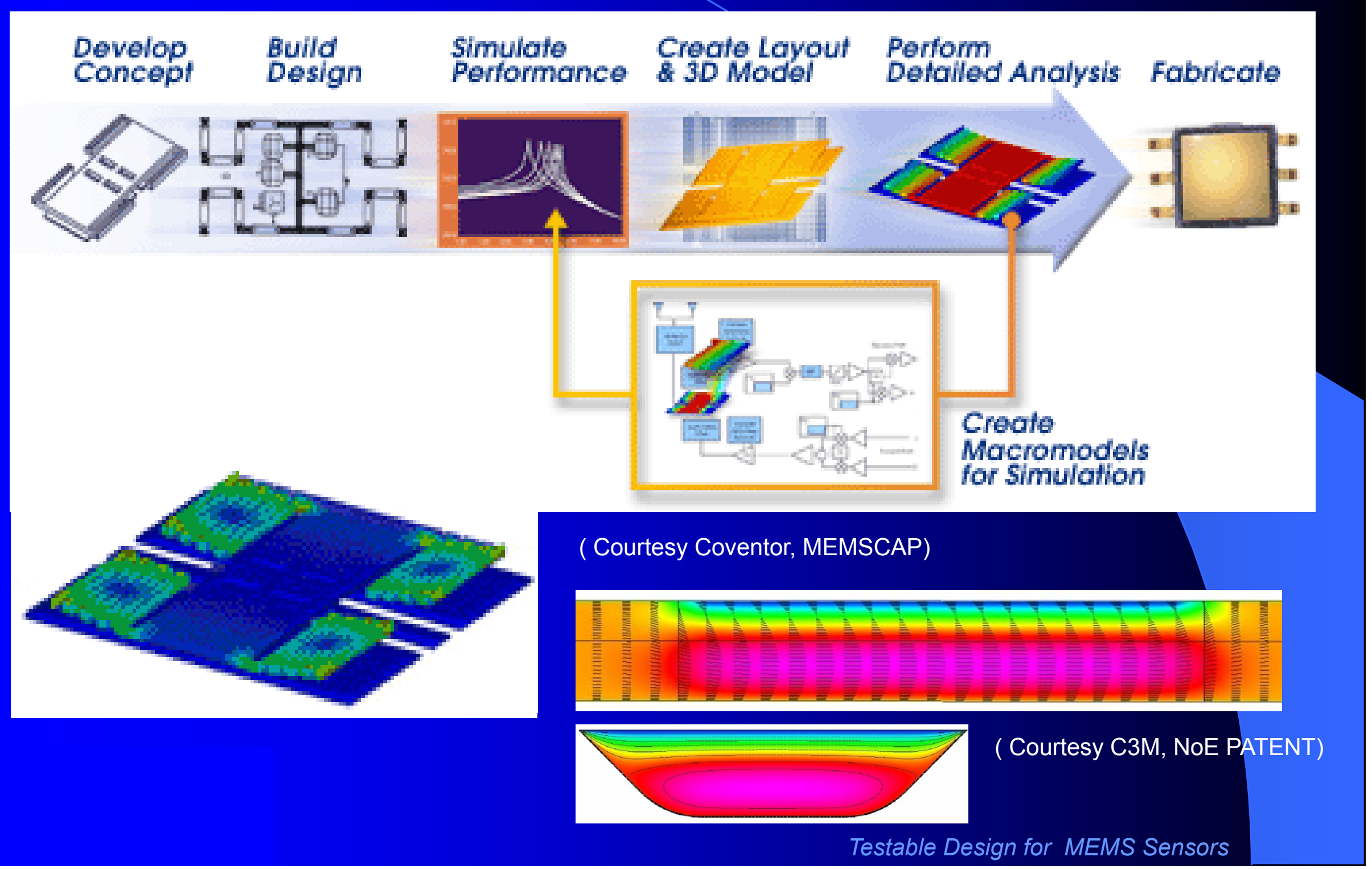




\section{Specification-Based MEMS Testing}

Optical MEMS: e.g. an optical switch

RF-MEMS: e.g. an RF switch

Inertial MEMS: accelerators

Flow \& Pressure MEMS: e.g. microphone

Fluidic MEMS: e.g. inkjet printer head + reliability 


\section{Generic Approach MEMS Testing}

e Electrical wafer tests: conventional ATE

- some started proprietary multi-domain wafer tests

Packaged full specification test: added special domain interfacing

- these are proprietary and not made public

Environmental tests: humidity, pressure, temperature - several general solutions possible

BASIC PROBLEM: obtaining high throughput And hence low costs ! 


\section{Optical MEMS: Optical Switch (1)}

\section{Basic operation \& MEMS implementation}

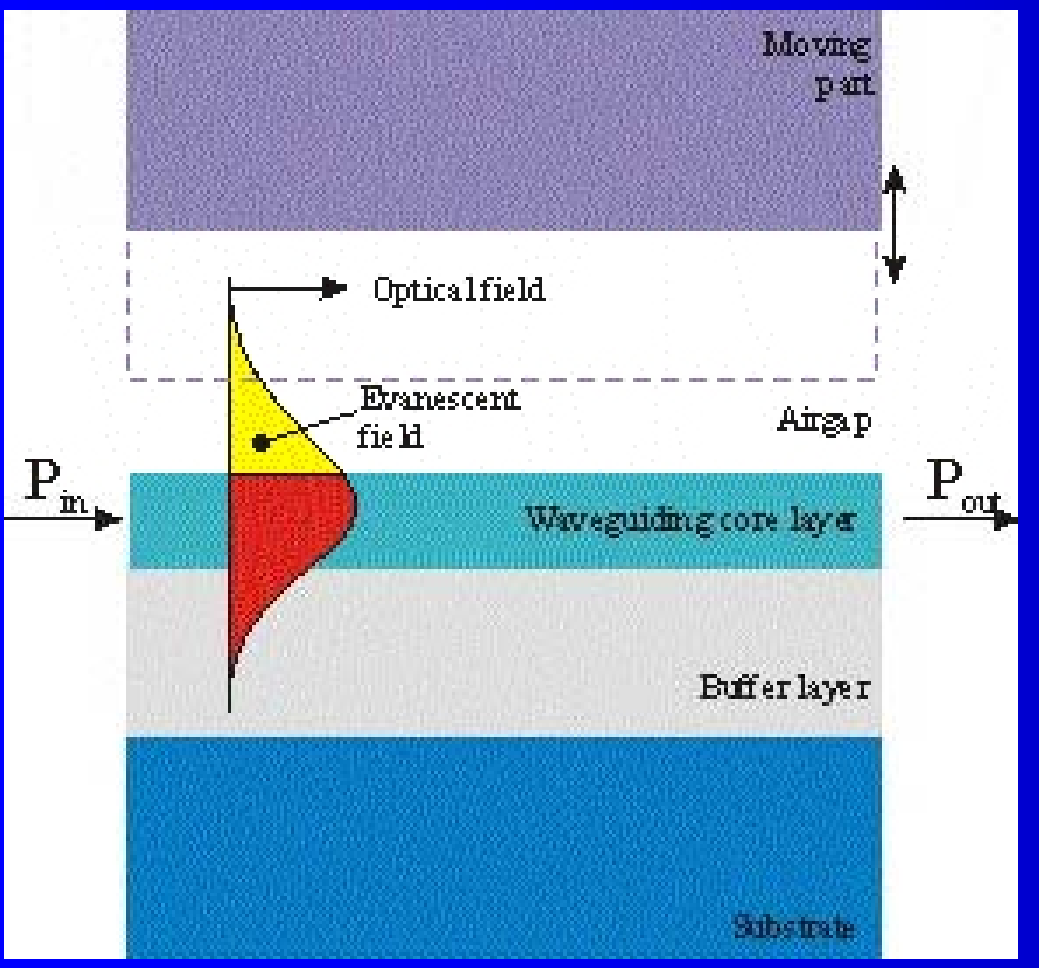

optical wafer

( Courtesy MESA+ IOMS)

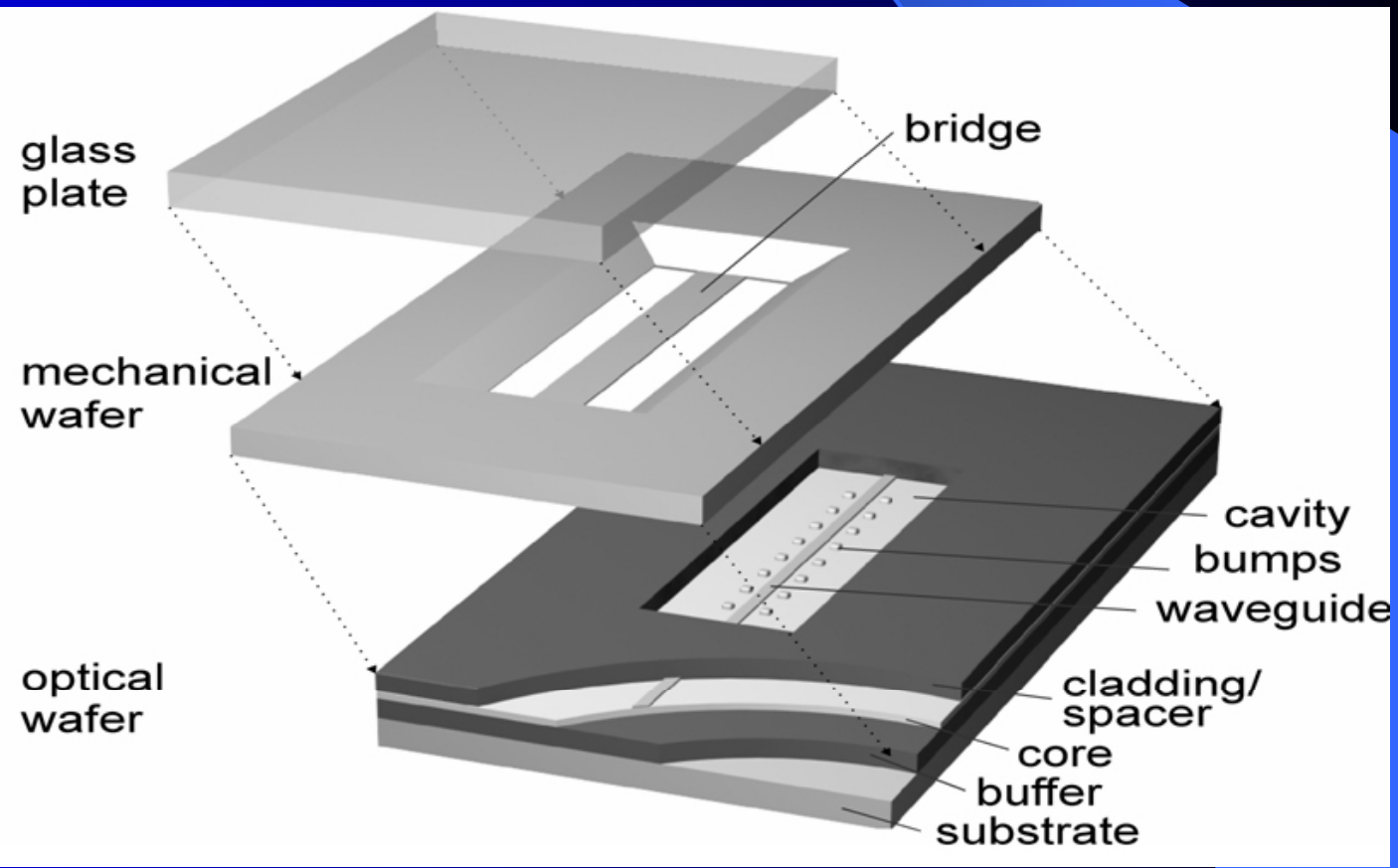

Testable Design for MEMS Sensors 


\section{Optical MEMS: Optical Switch (2)}

Bulky test environment \& functional parameter tests

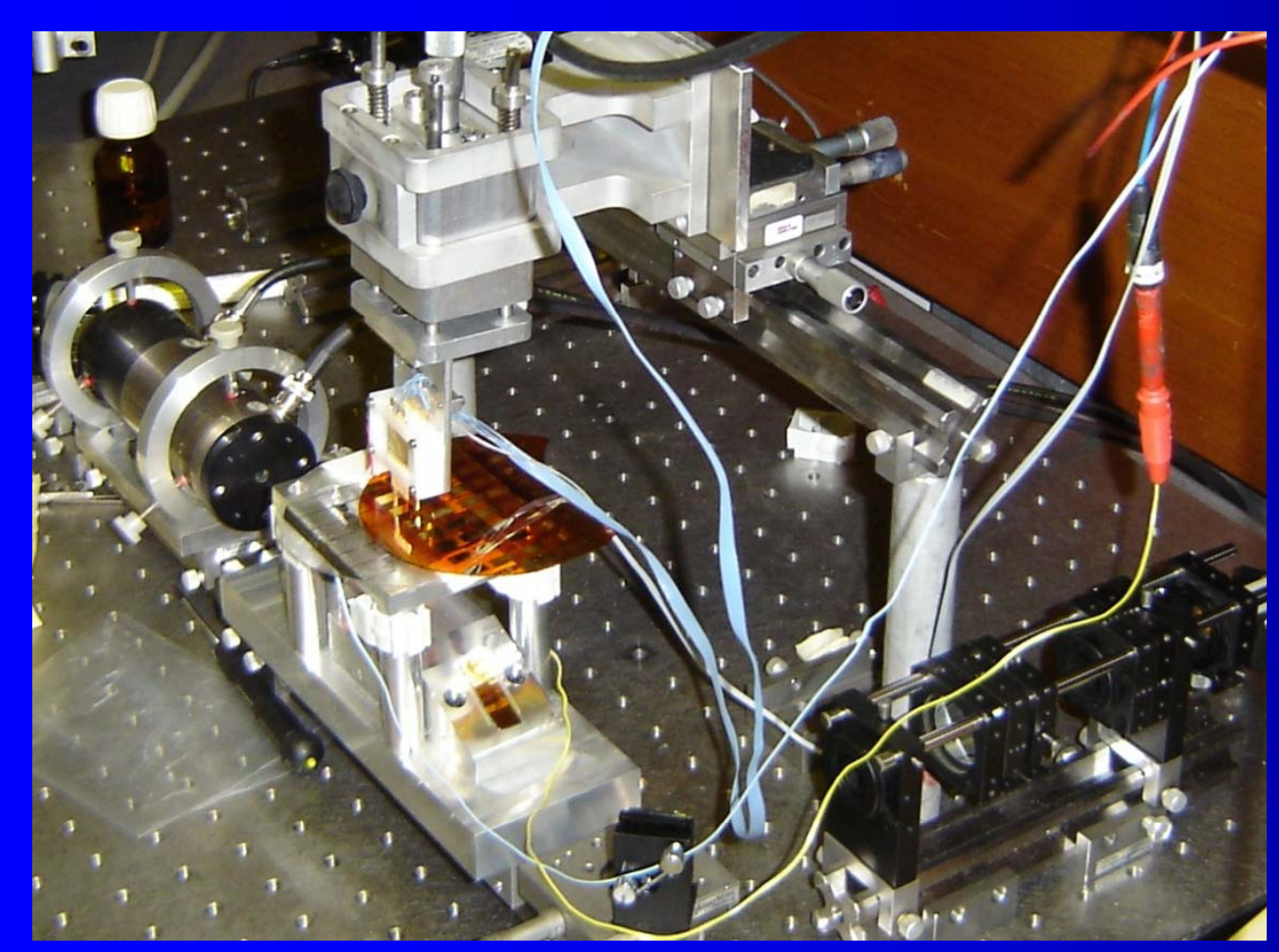

Test set-up

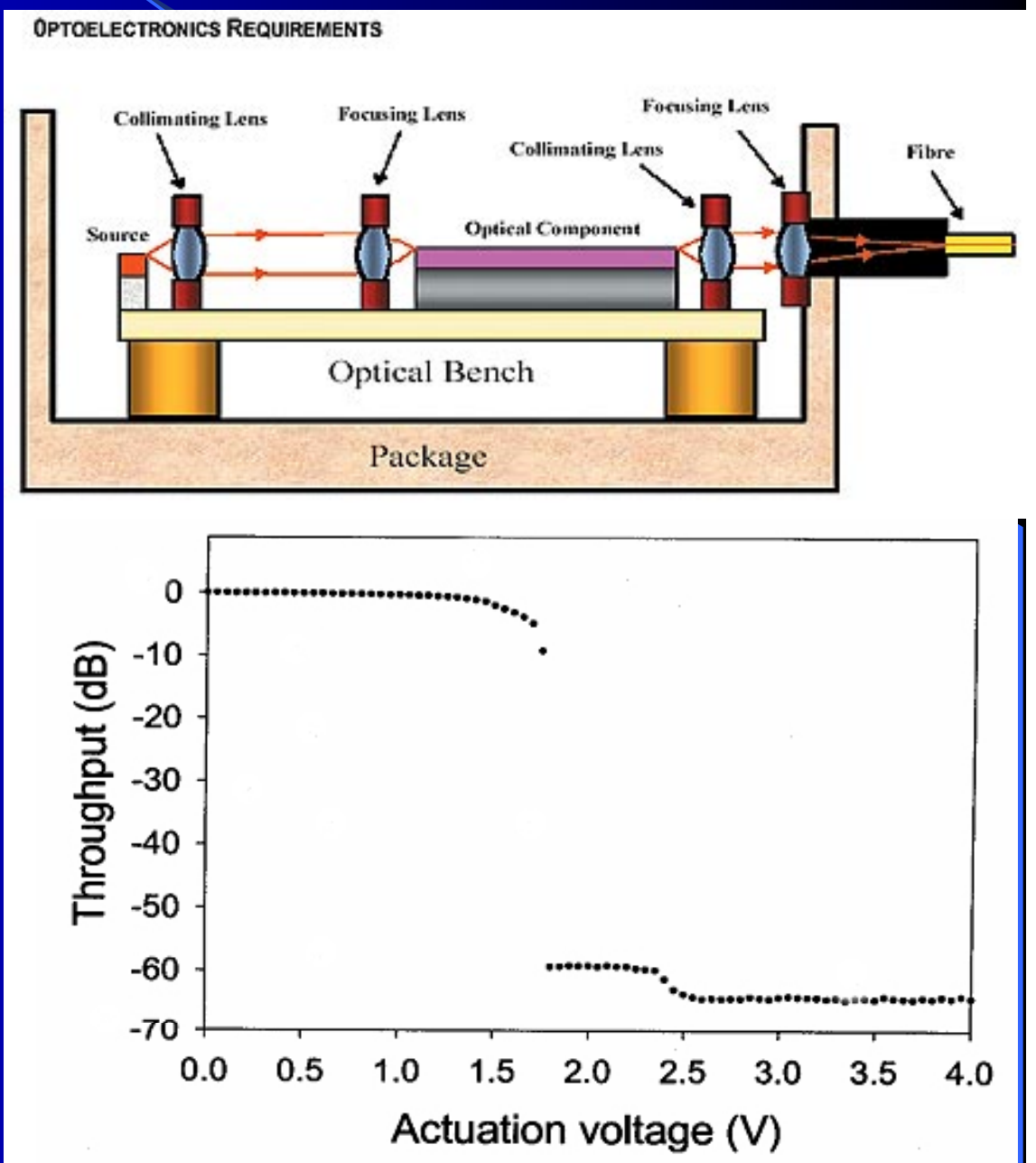

Results 


\section{Optical MEMS: Optical Switch (3)}

- Many defects unvisible during specification tests

- laserdiodes \& opto diodes could be used for DfT/BIST
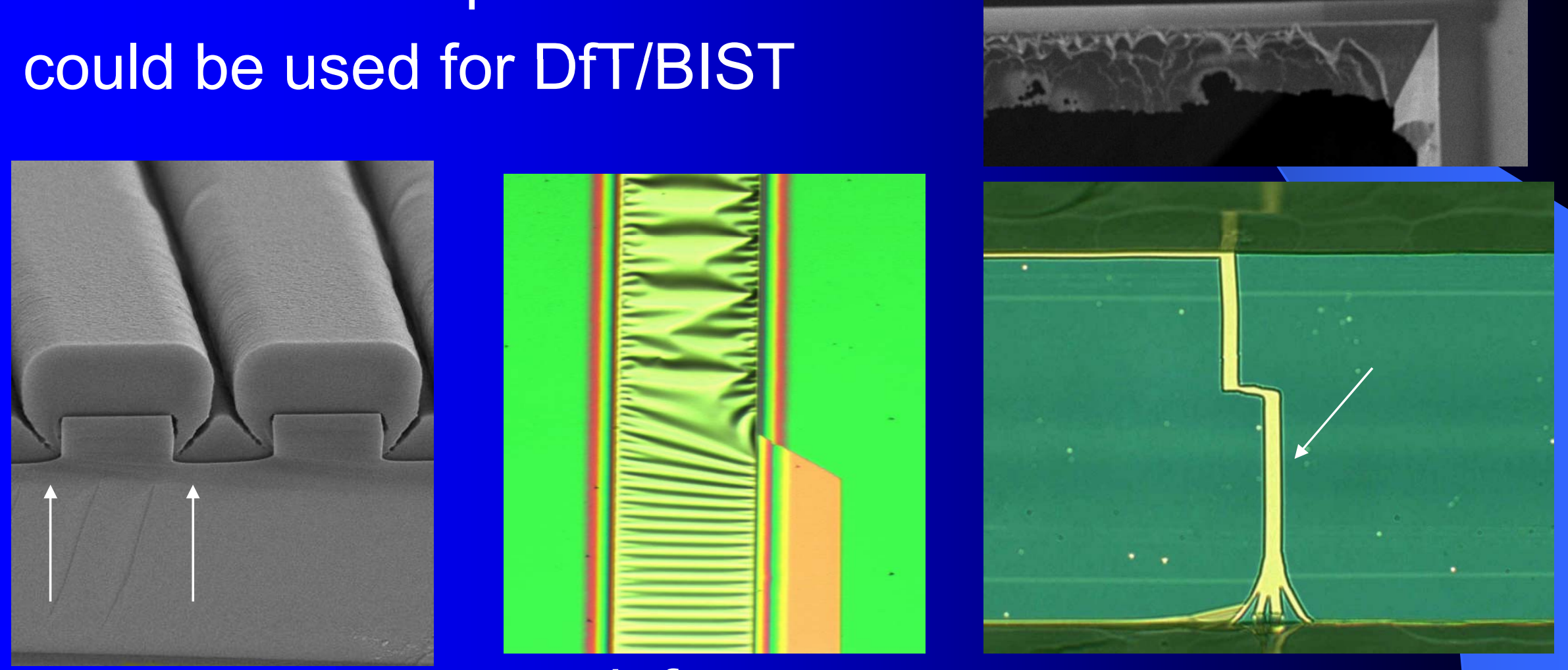

defects 


\section{Testing MOEMS: Optical Switch (4)}

- Different specialzed ATE available. Opto relatively easy to convert
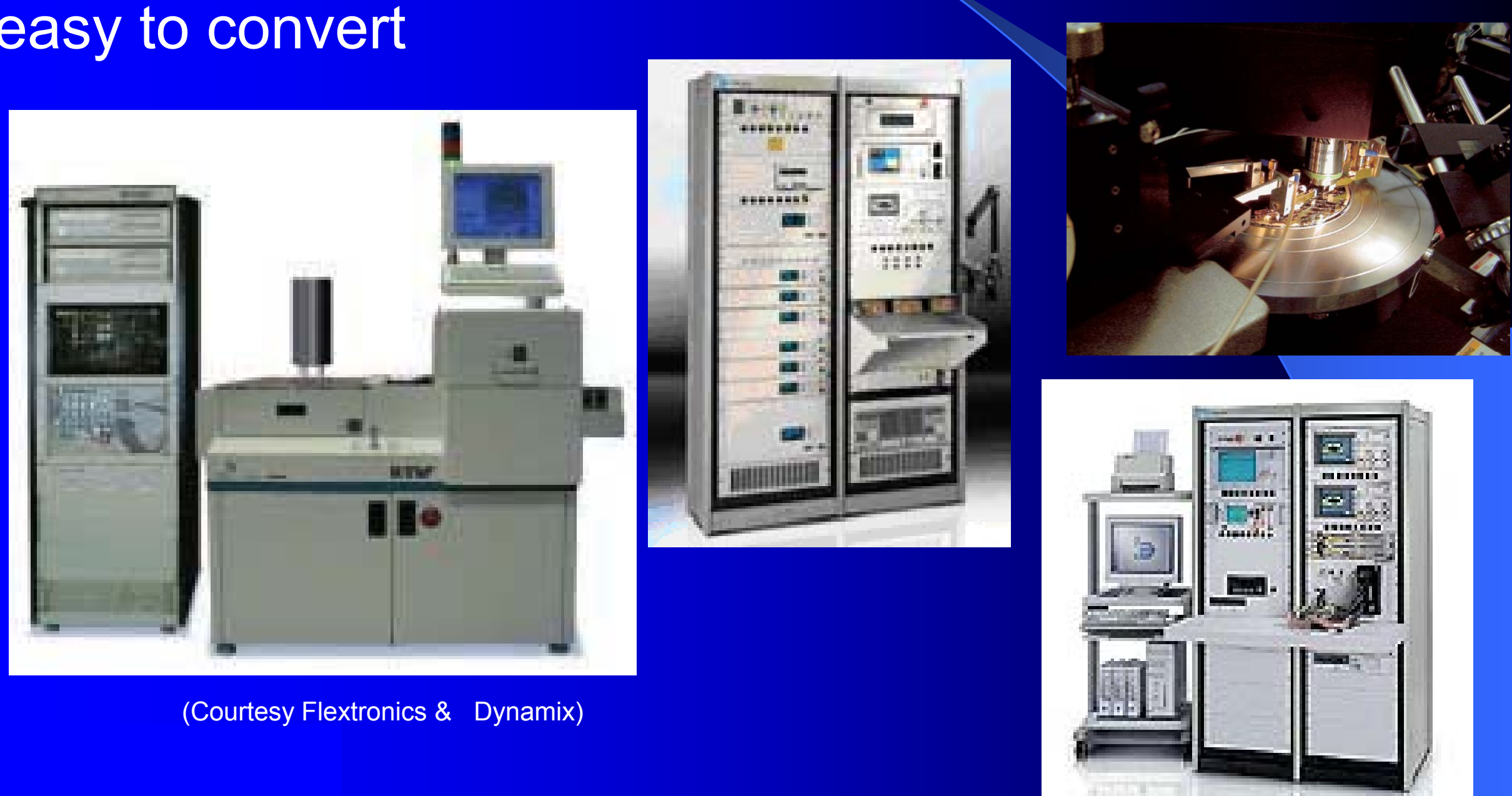

(Courtesy Flextronics \& Dynamix) 


\section{Testing MEMS-based RF Switches (1)}

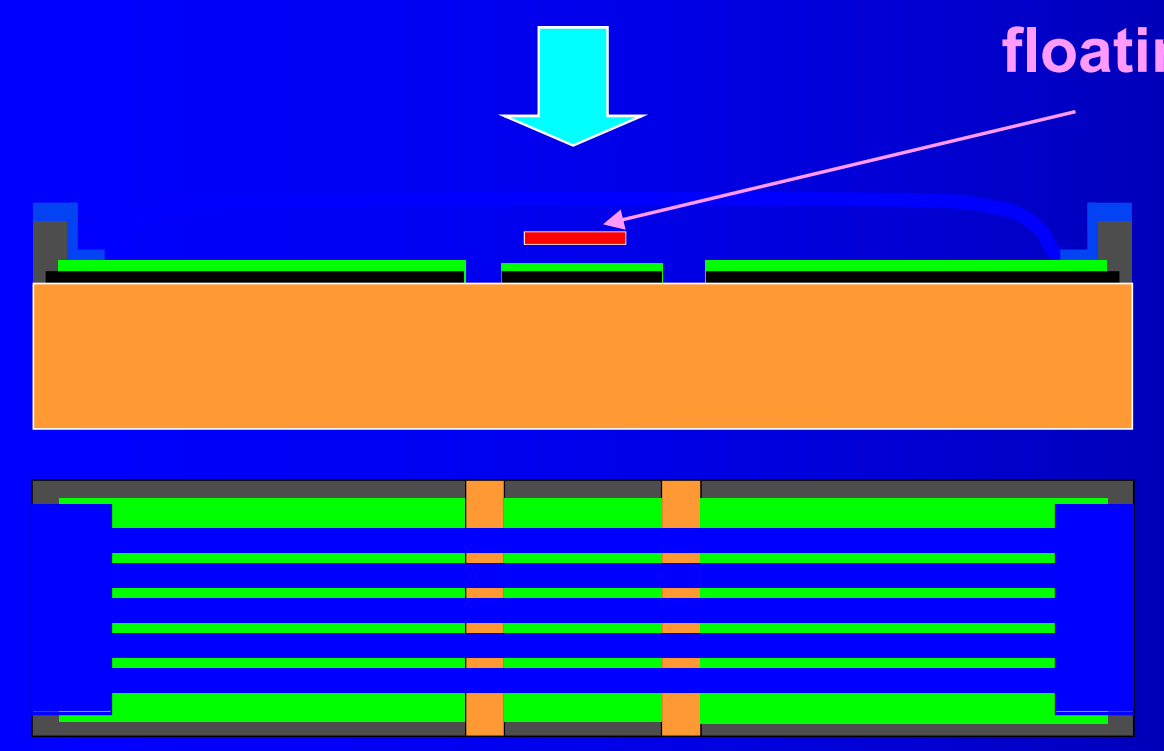

ing top metal
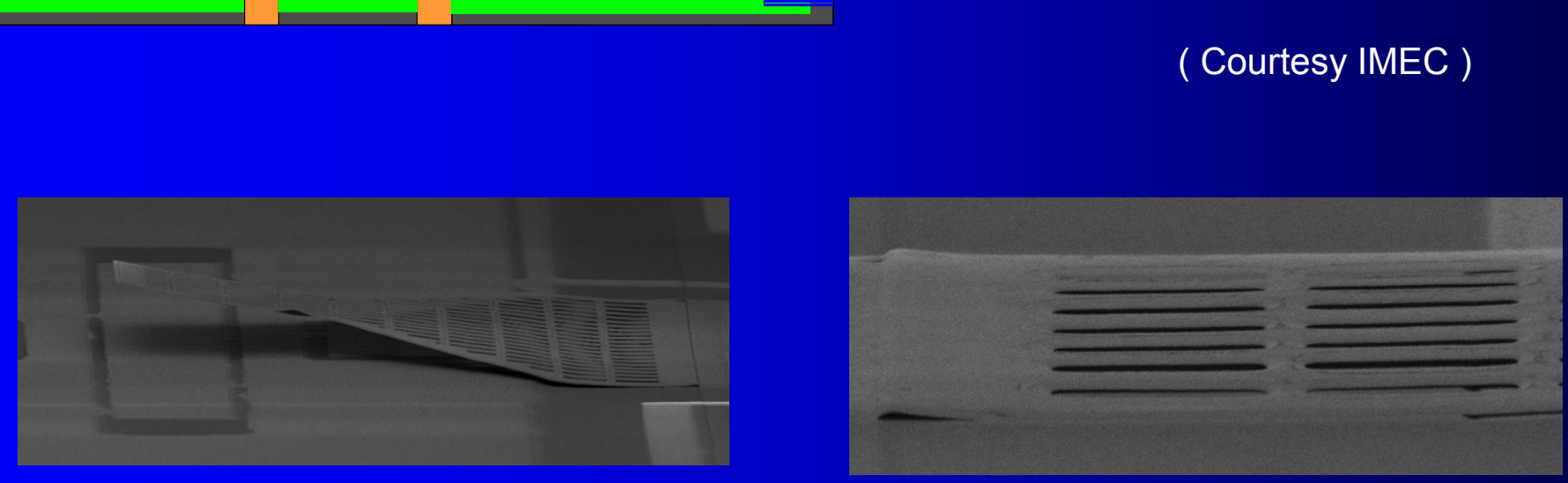

Defects: (stiction, stress) 


\section{Influence of Packaging}
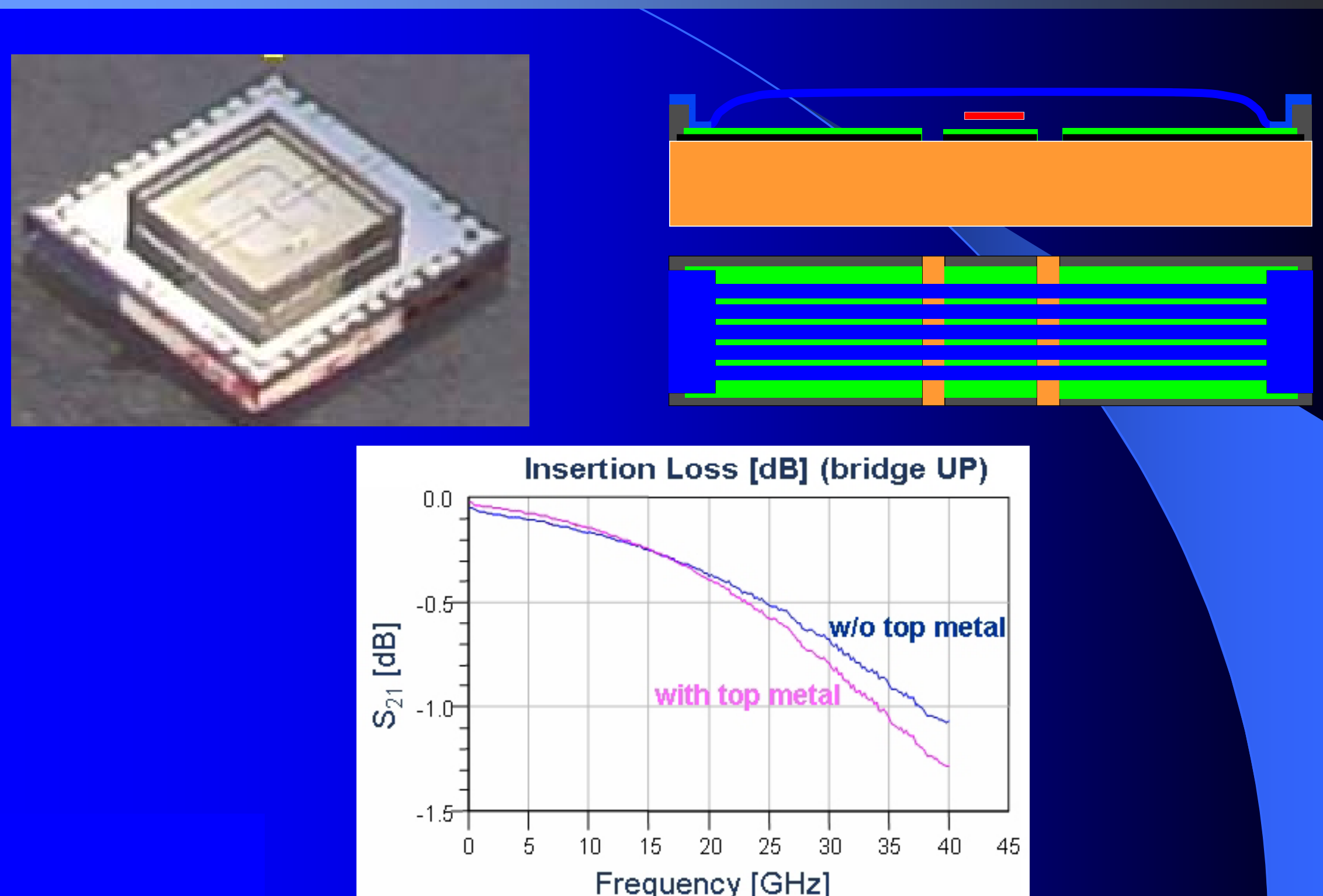

Testable Design for MEMS Sensors 


\section{Testing MEMS-based RF Switches (2)}

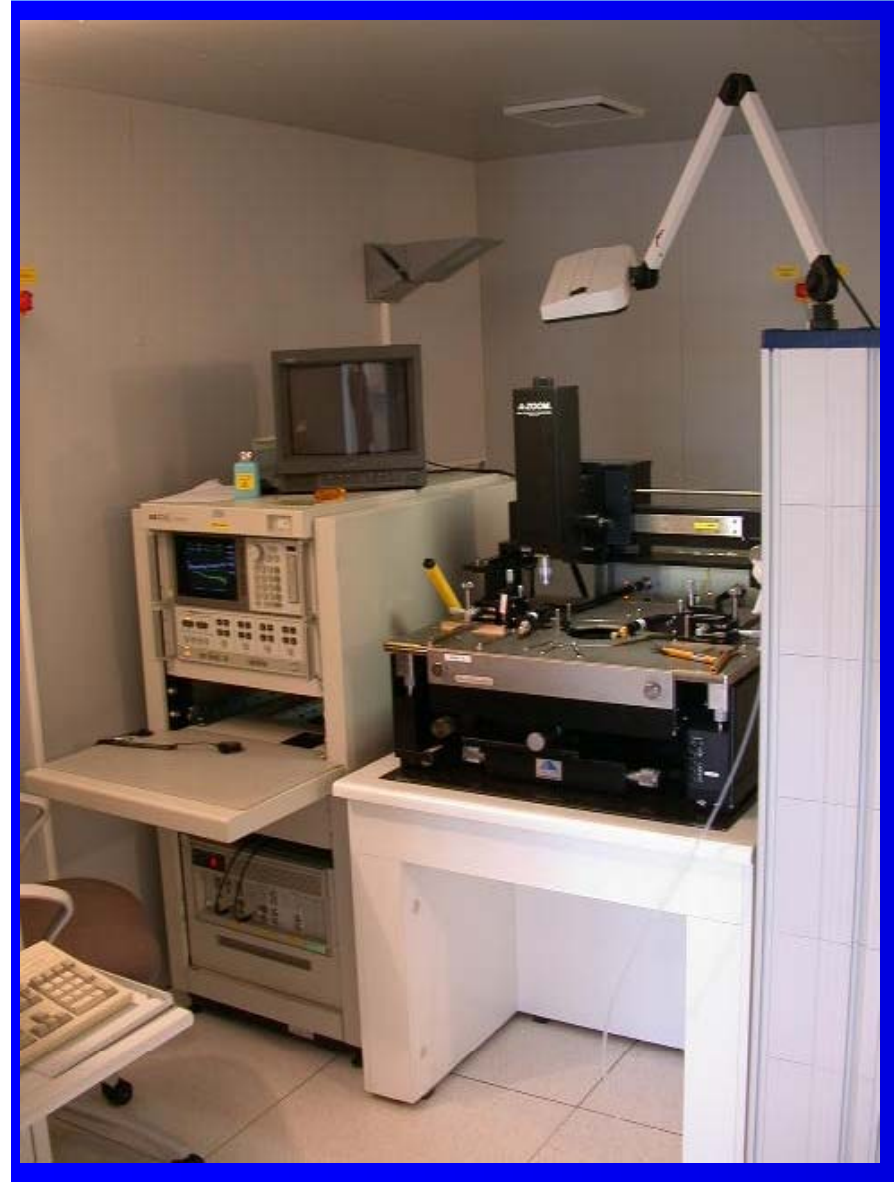

( Courtesy IMEC )

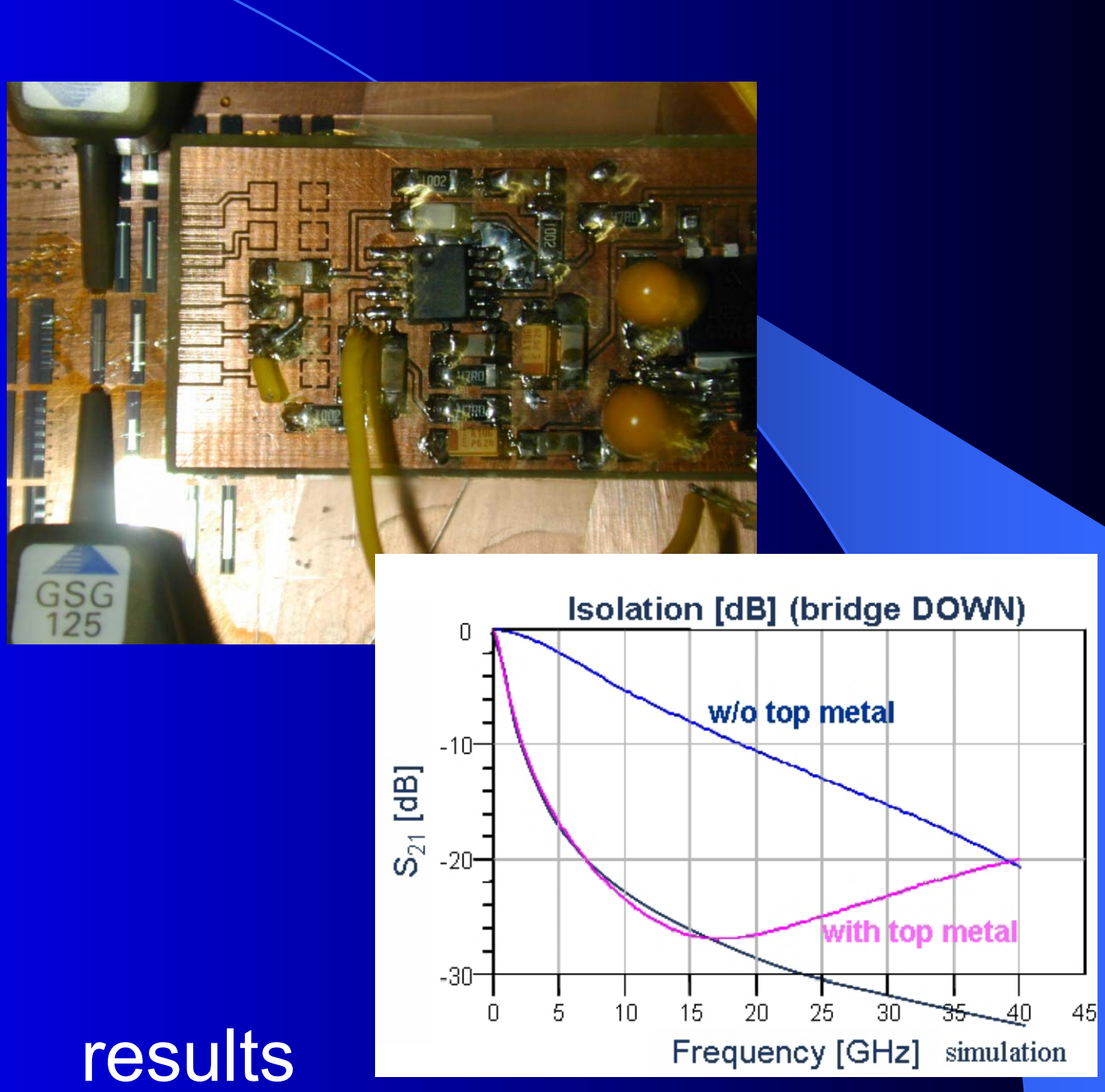

Testable Design for MEMS Sensors 


\section{MEMS-based RF Switches}

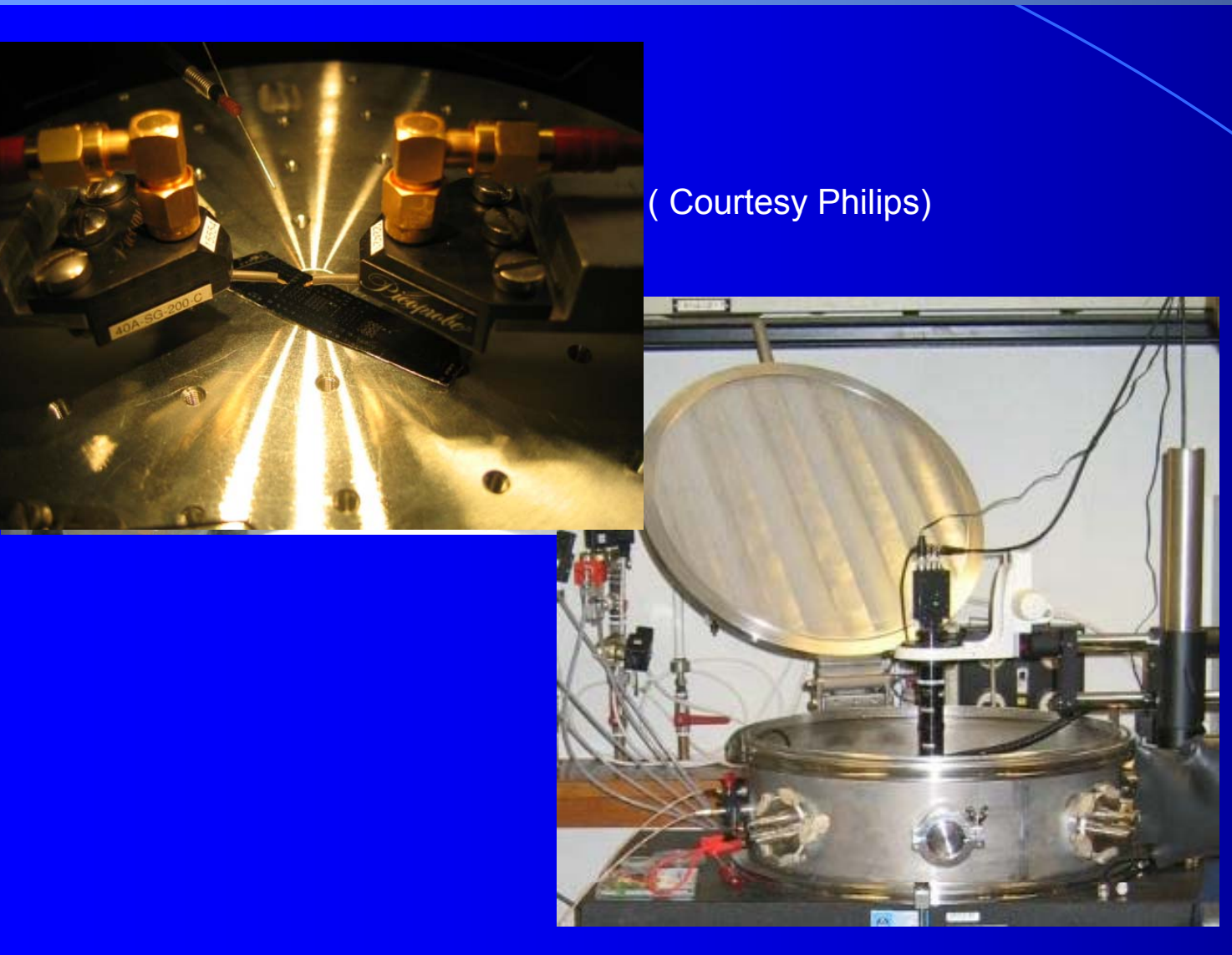

Humidity \& Pressure Test Setups

OV

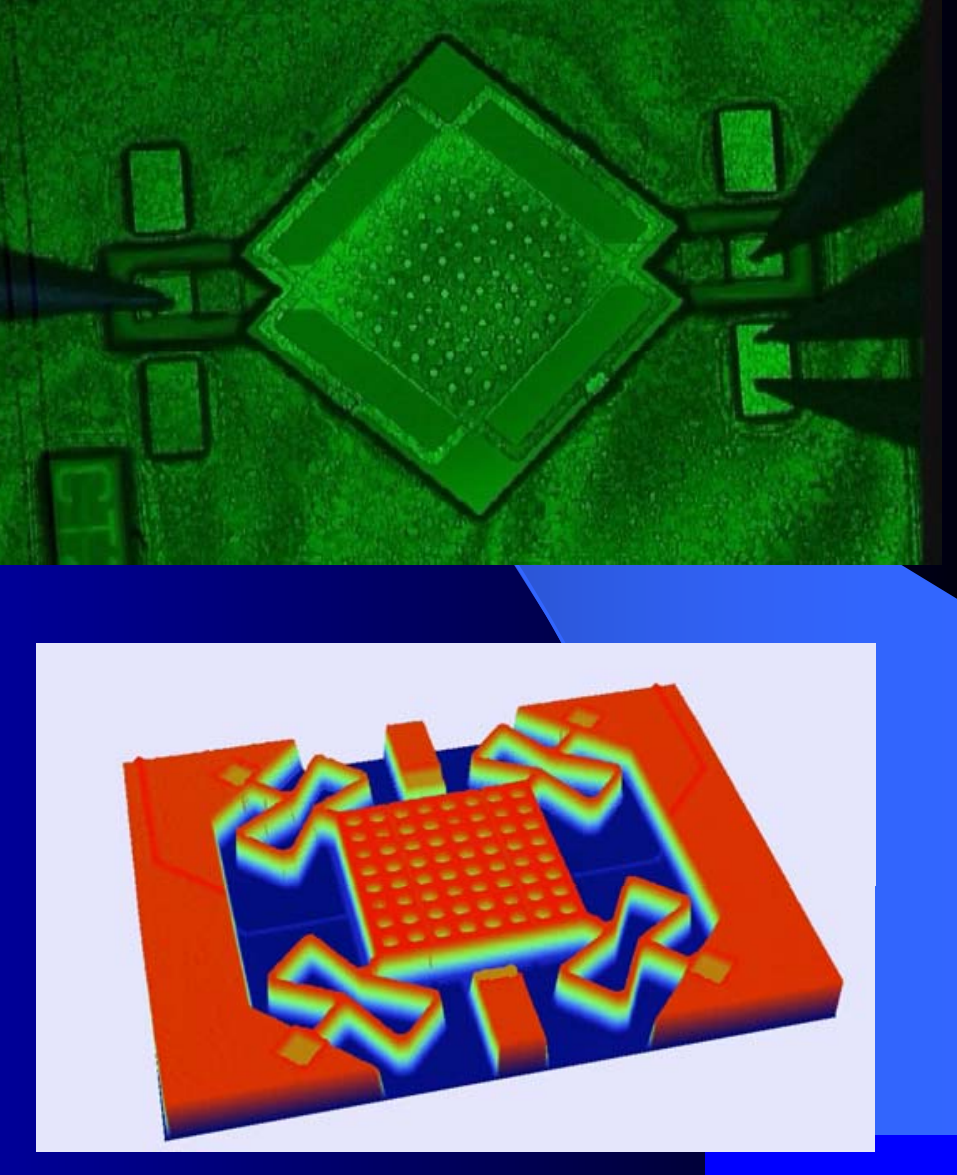

Simulation \& interference test 


\section{Measurements in Nitrogen \& ATE}
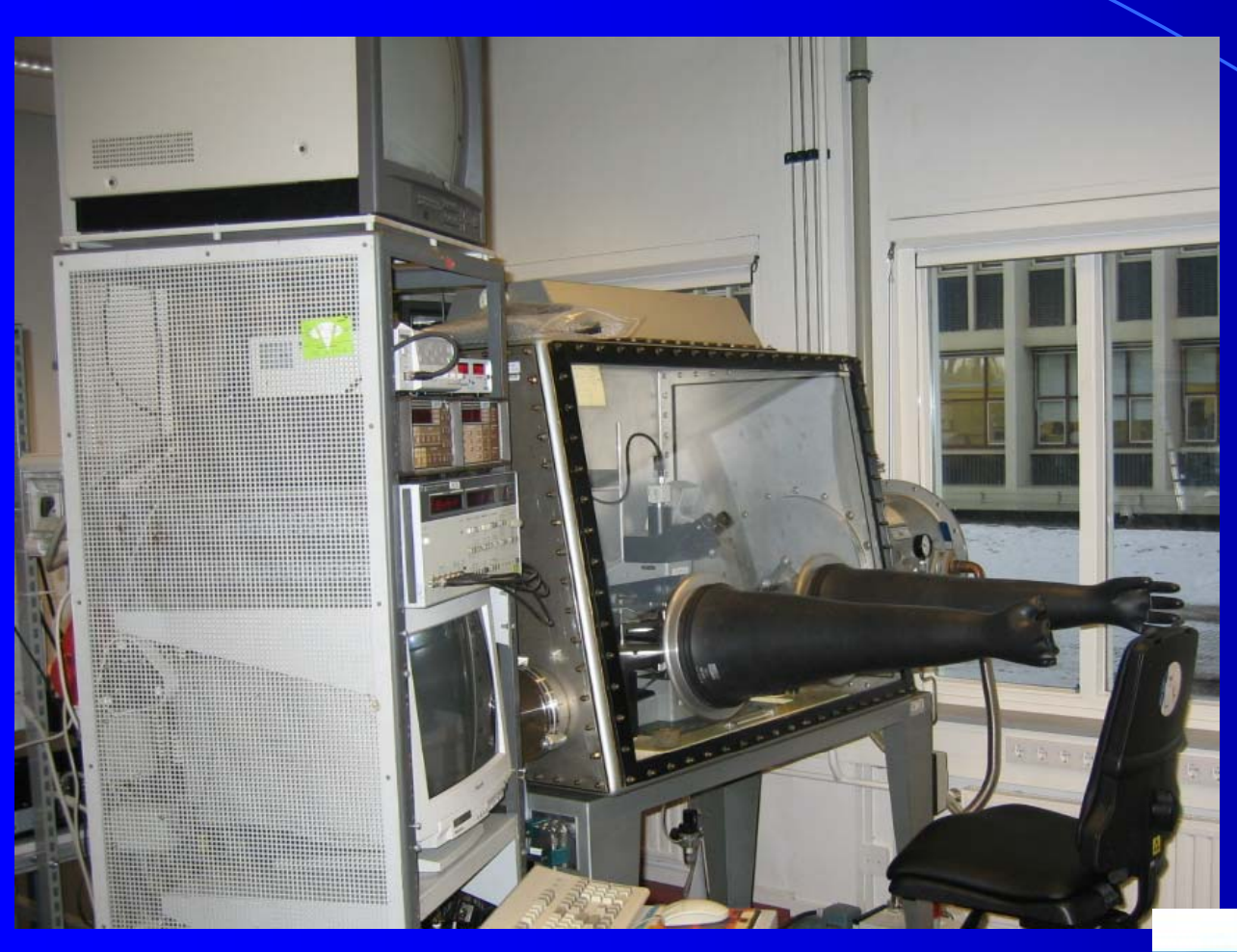

Fixed humidity

( Courtesy Philips)
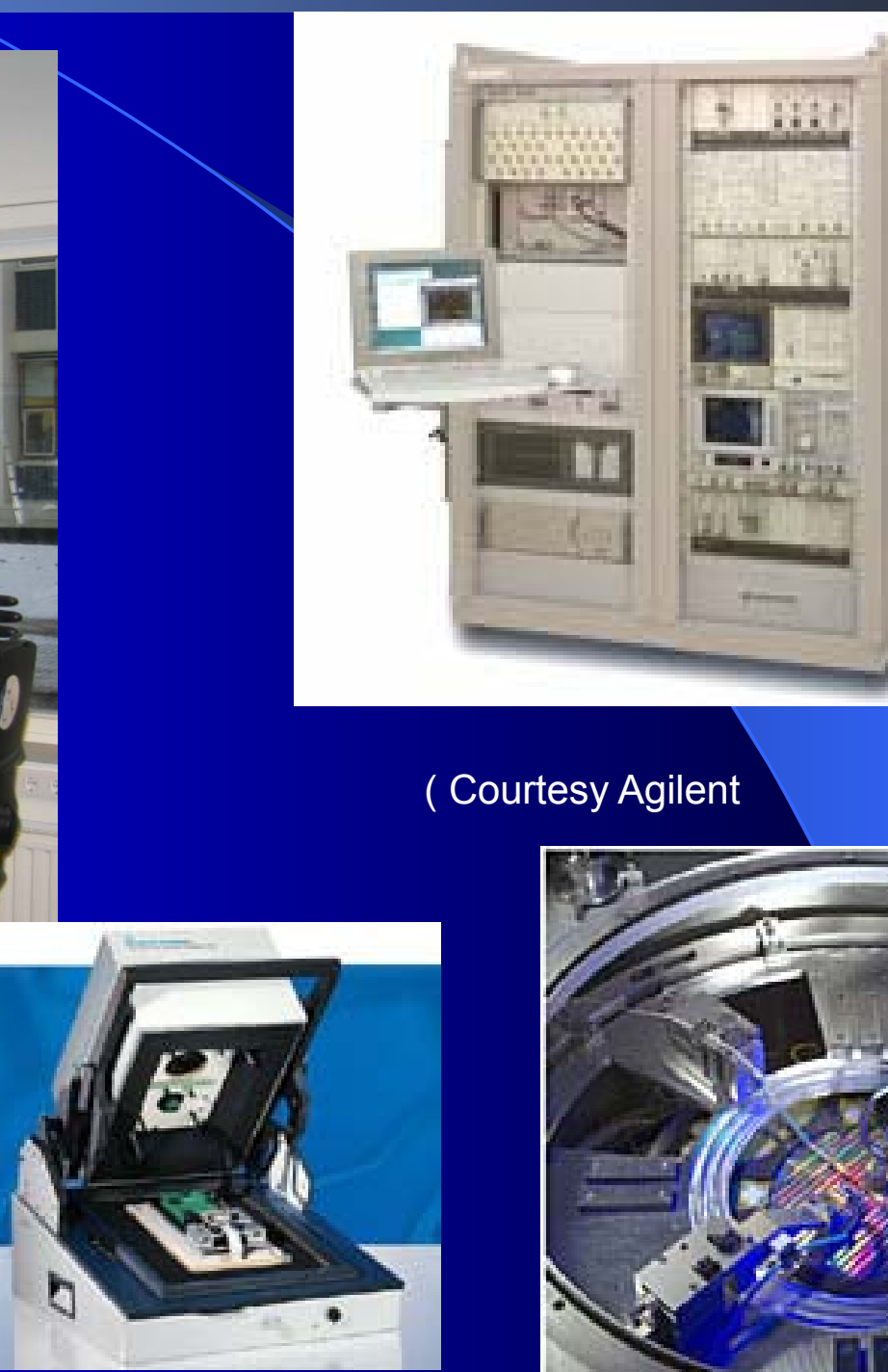

Rhode Schwartz \& Suss)

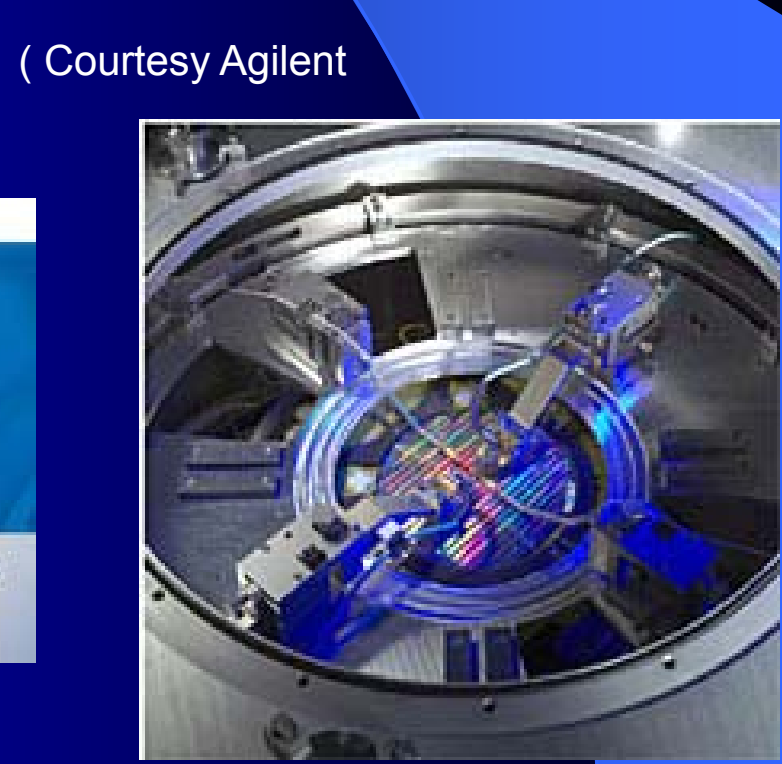

Testable Design for MEMS Sensors 


\section{Multisite RF Antennas Testing}

MEMS-based antenna \& Schottky diode
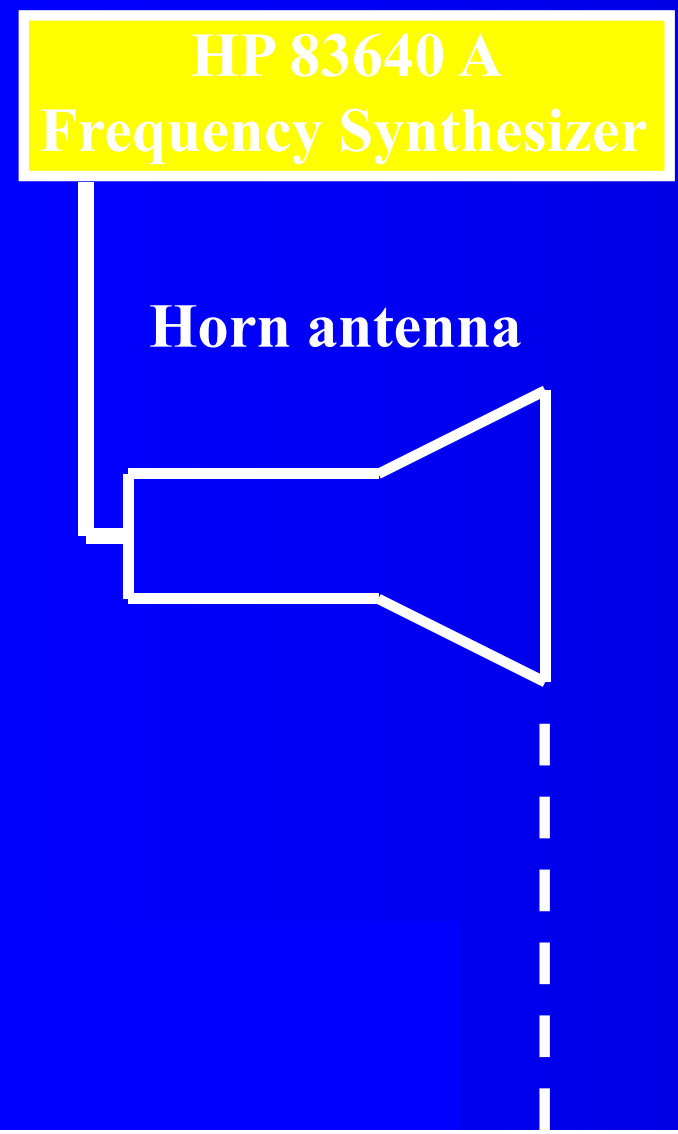

\section{a}

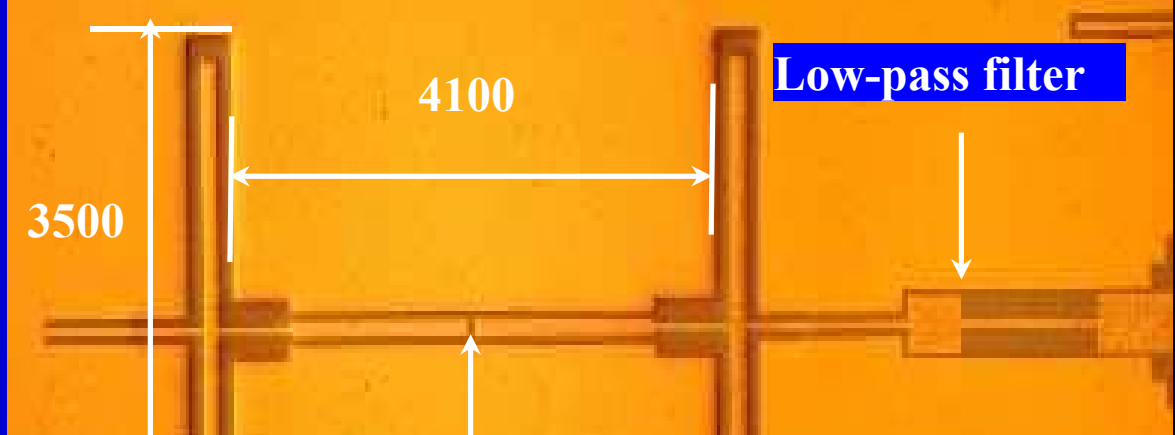

E plane

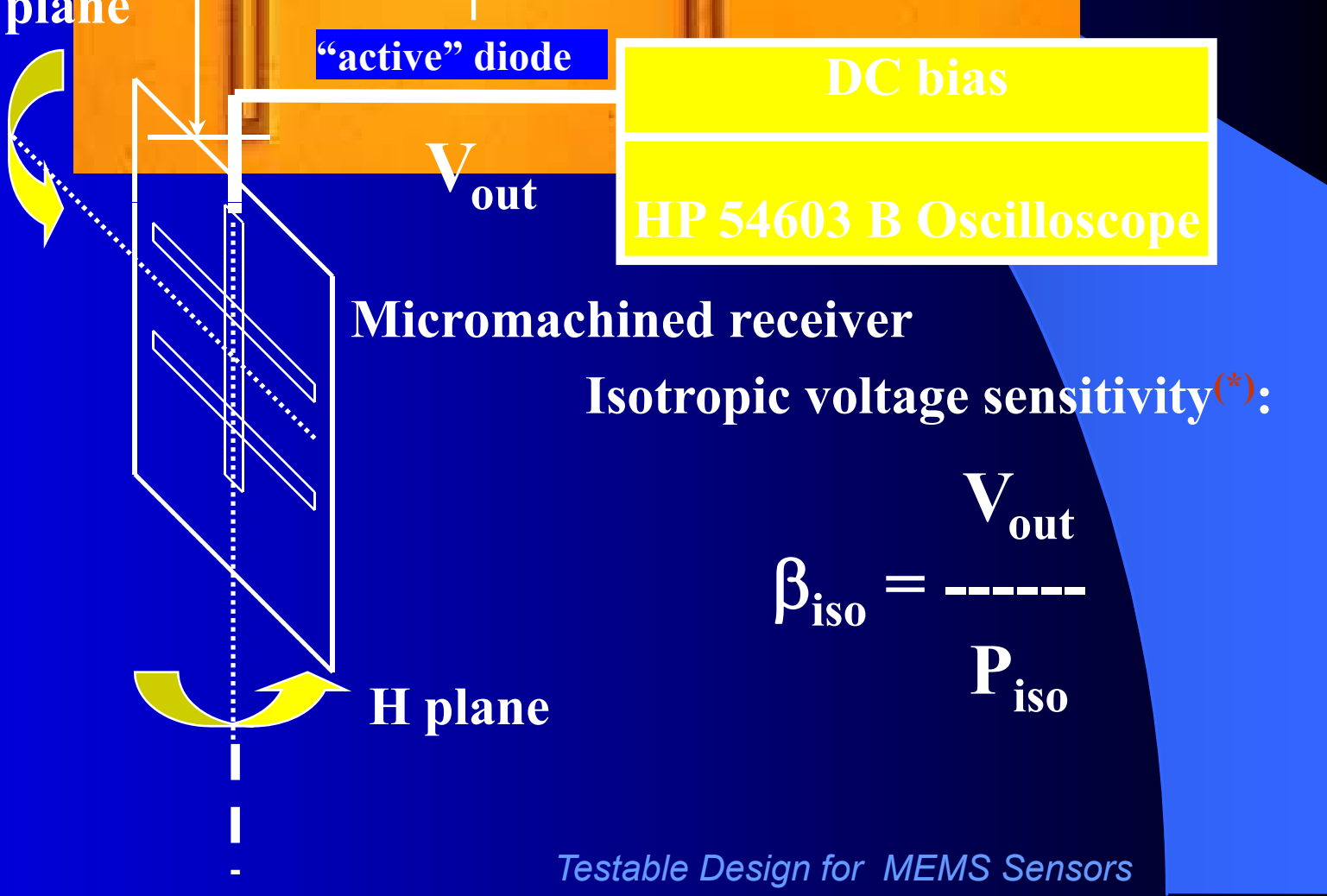




\section{Inertial MEMS: Accelerators, Gyros 1}

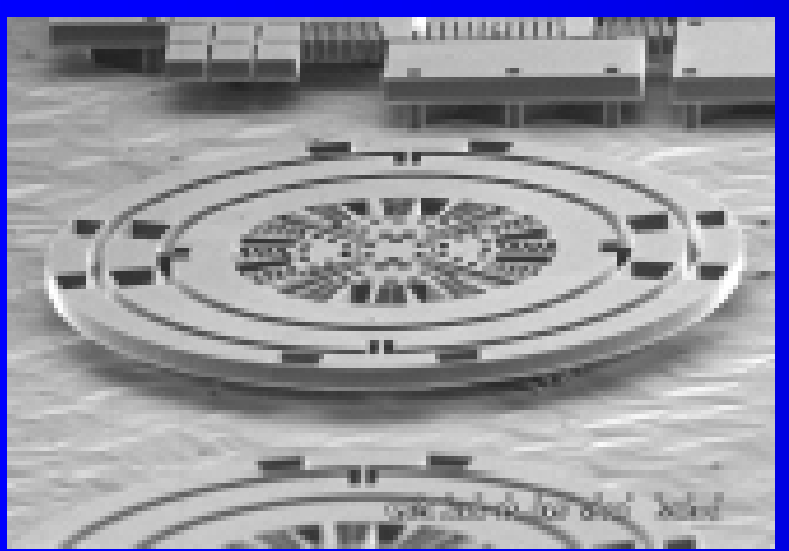

\section{Some accelerometers}

Functional tests: e.g. resonator $f$ and sensitivity \& offset calibration
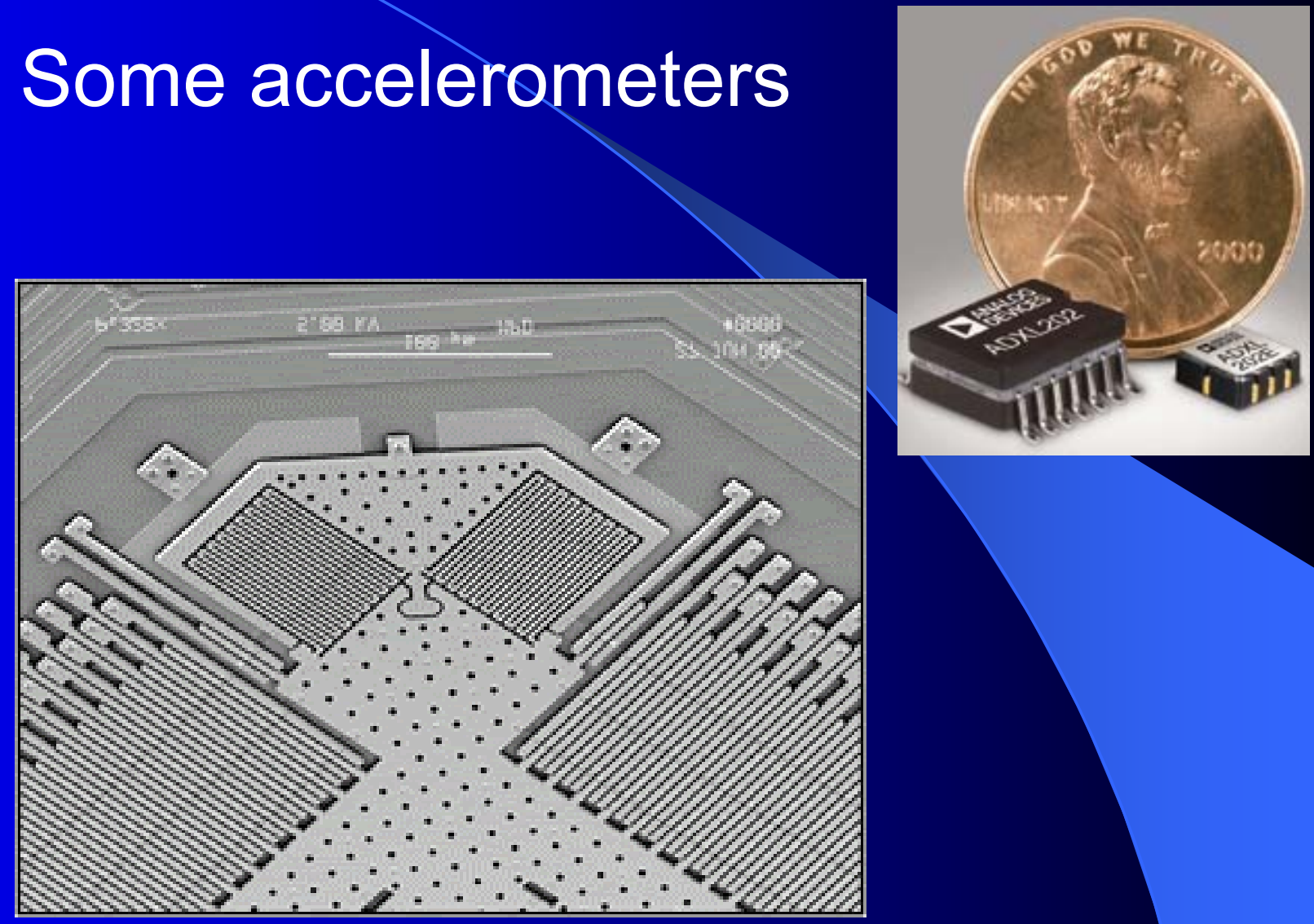

Stimuli: g, frequency, environment: $\mathrm{T}$ and $\mathrm{RH}$ 


\section{Inertial MEMS: Accelerators, Gyros 2}
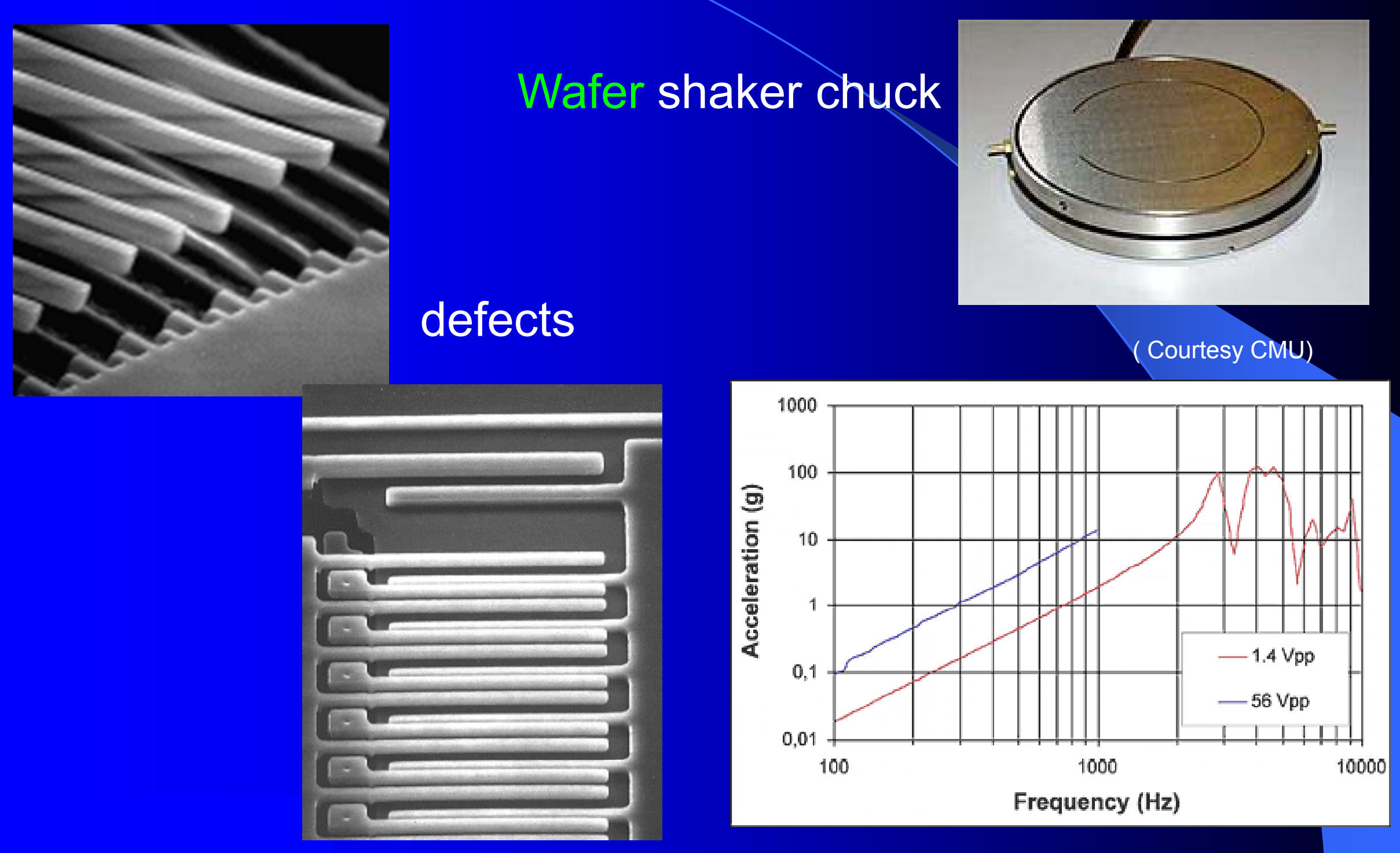


\section{Inertial MEMS: Production Test}
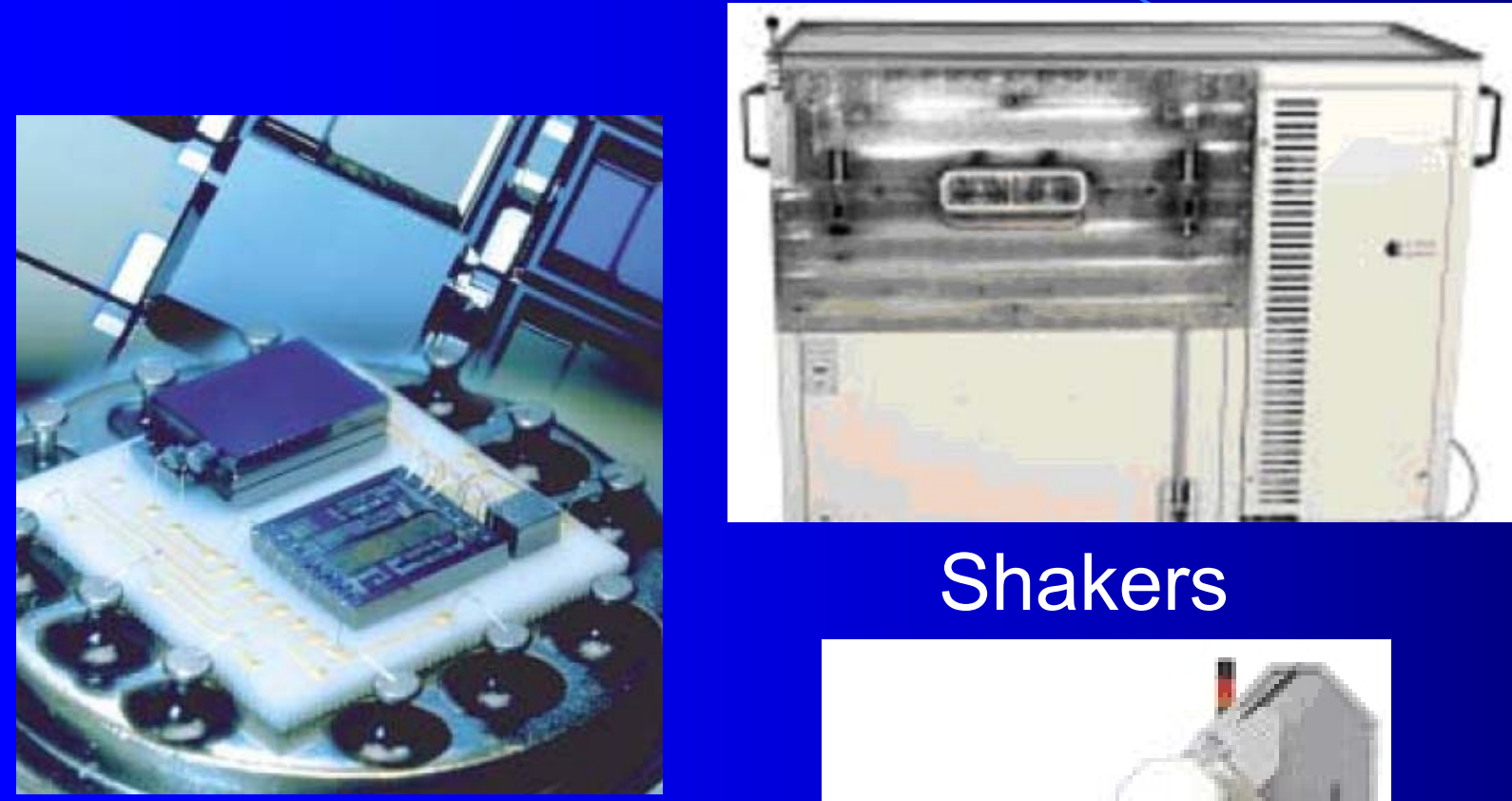

Shakers

Large established market:

$>100$ million parts annually
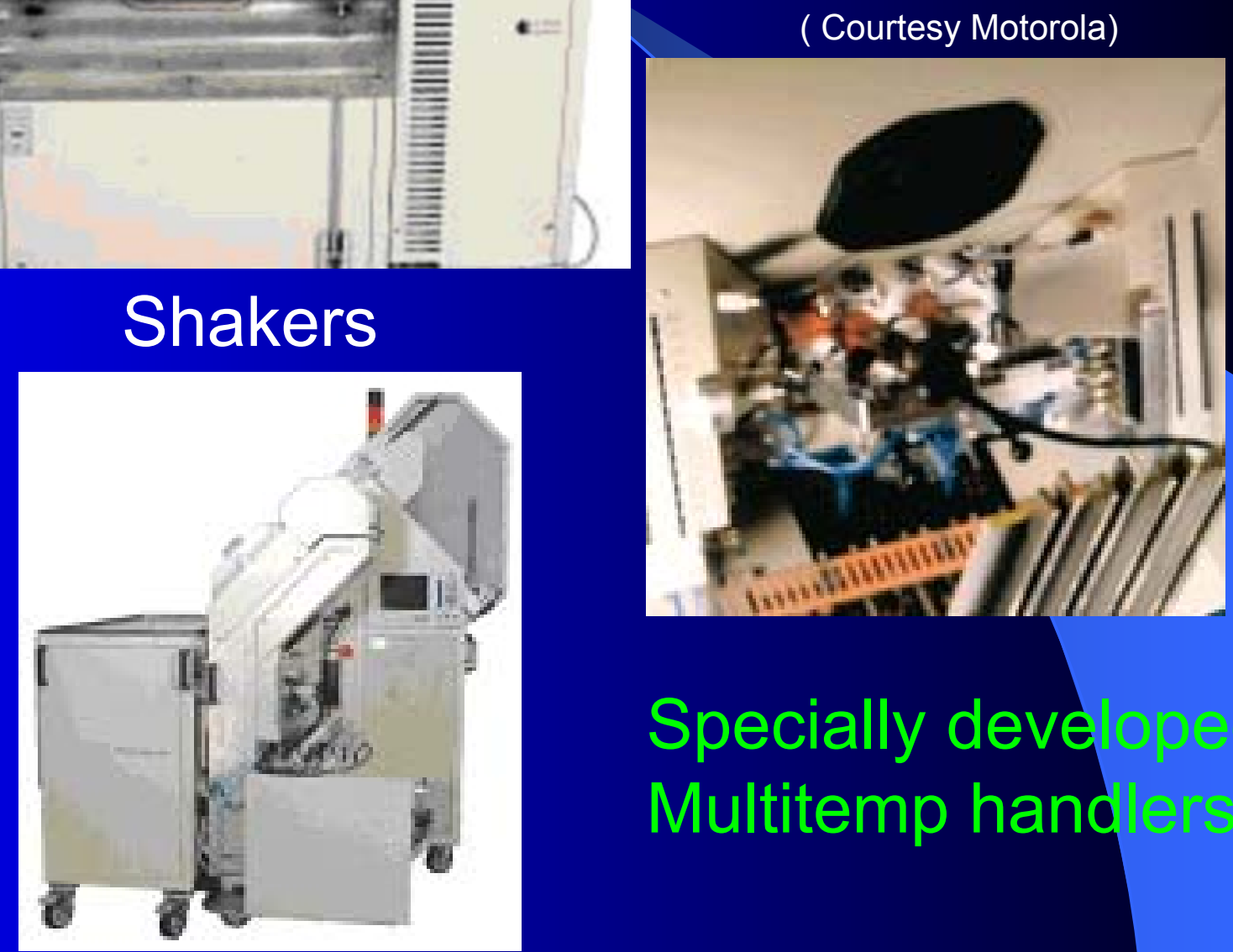

Gravity handlers: g, frequency, $T$ 


\section{Inertial MEMS: Structural Testing}

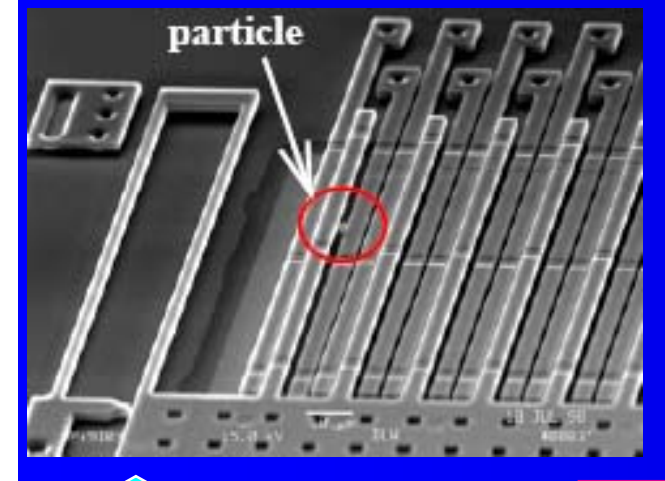

1. Defect

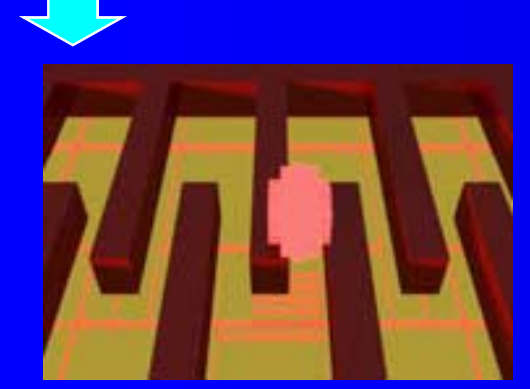

Defect model

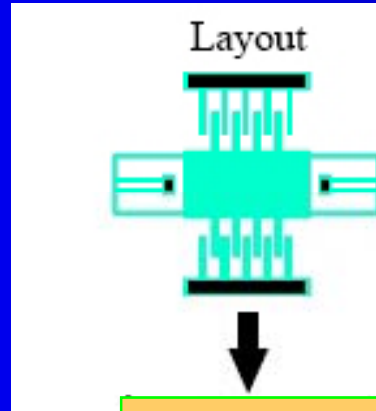

caramel
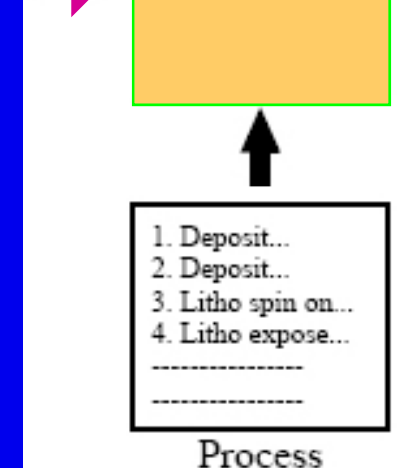

Process
Electrical

simulation
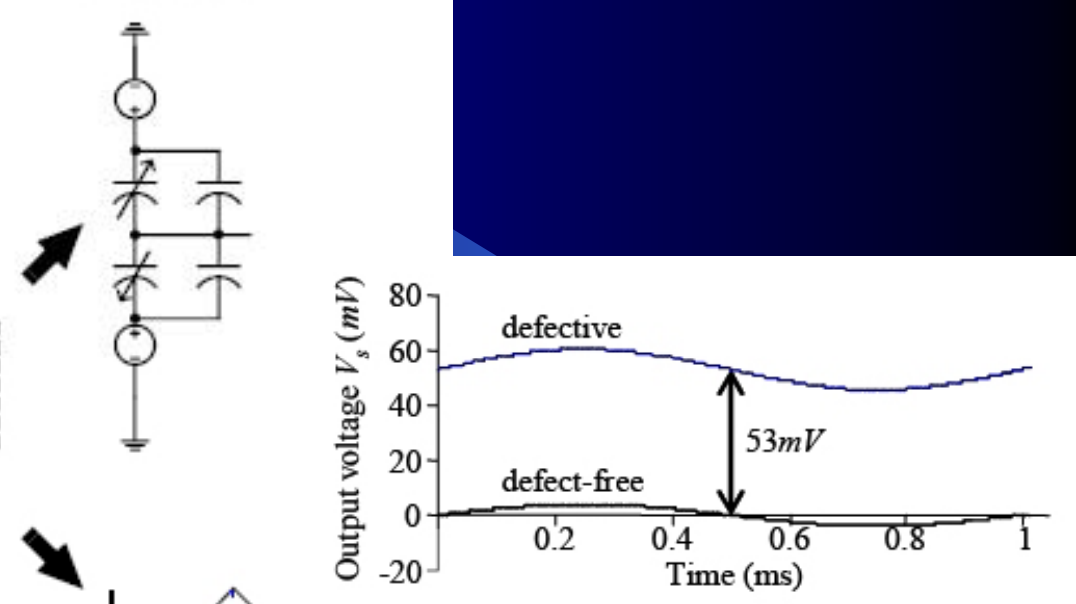

Output from test

( Courtesy CMU)

Do sensitivity analysis (A,f, etc) defect detection -> ATPG Add DFT if required for enhanced fault coverage 


\section{MEMS-based Flow Microsystem (1)}

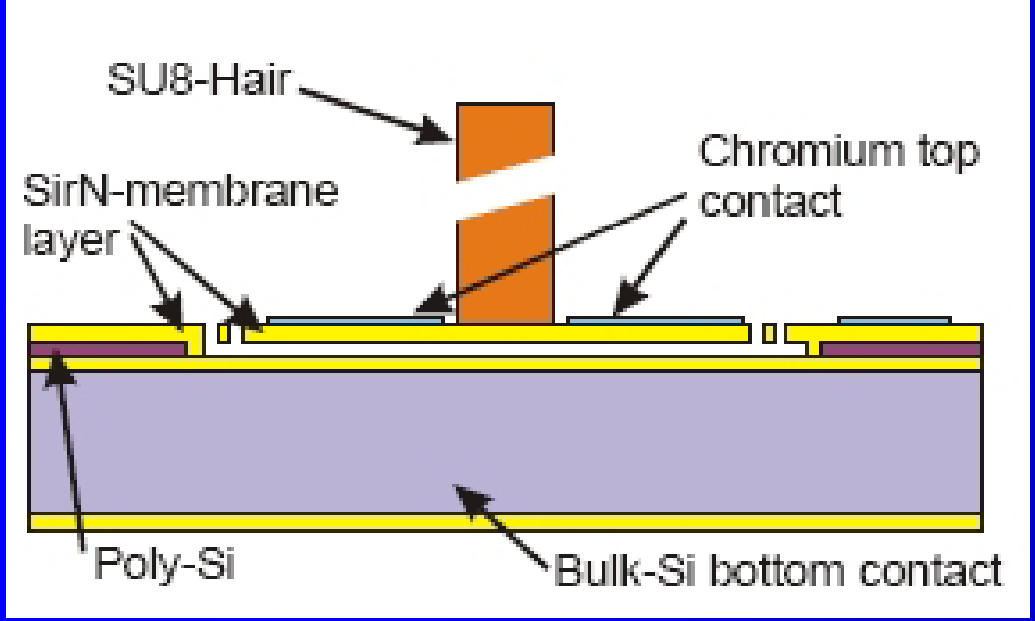

( Courtesy MESA+ TST)
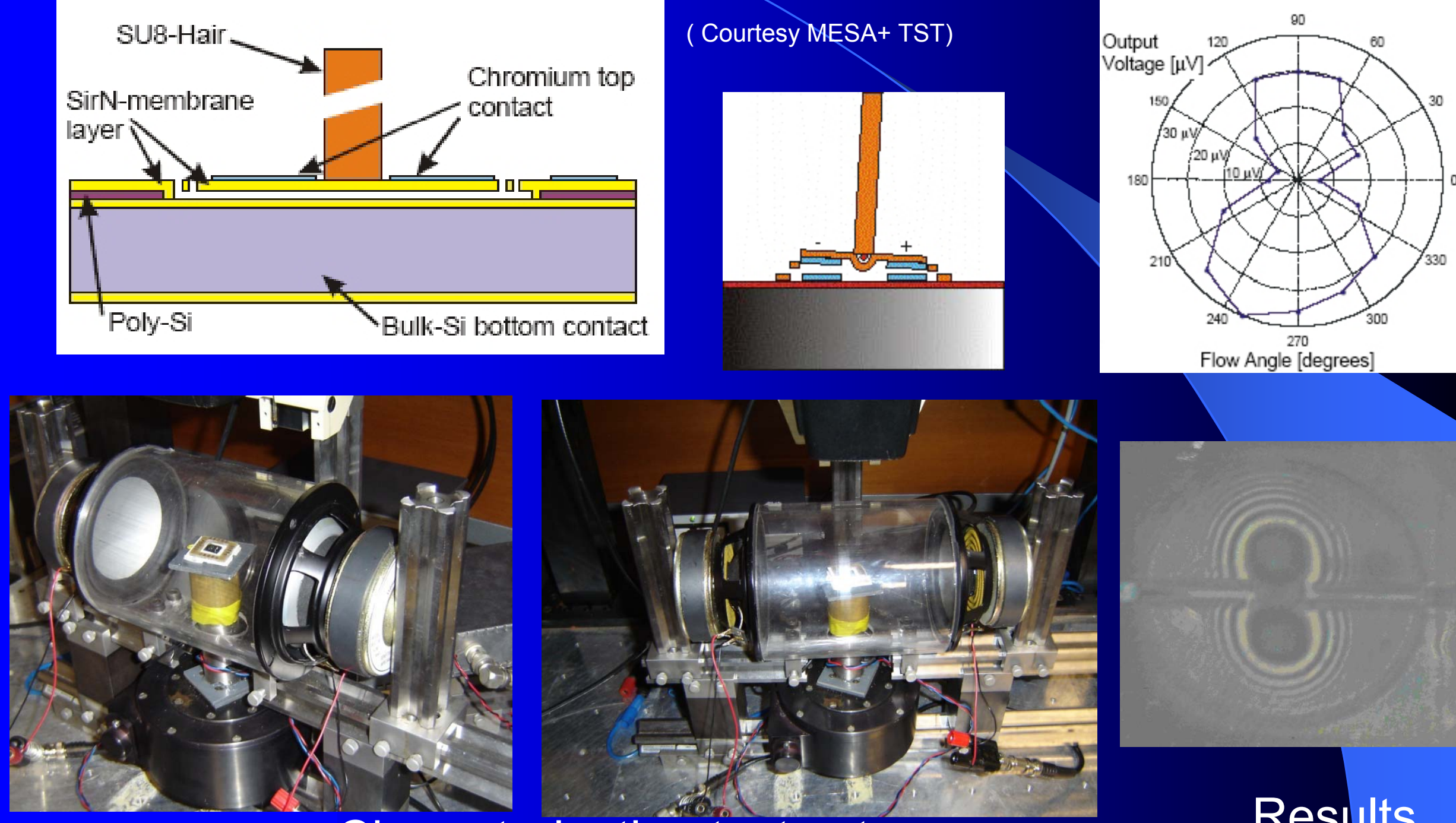

Characterization test set-up

Results 


\section{MEMS-based Flow Microsystem (2)}
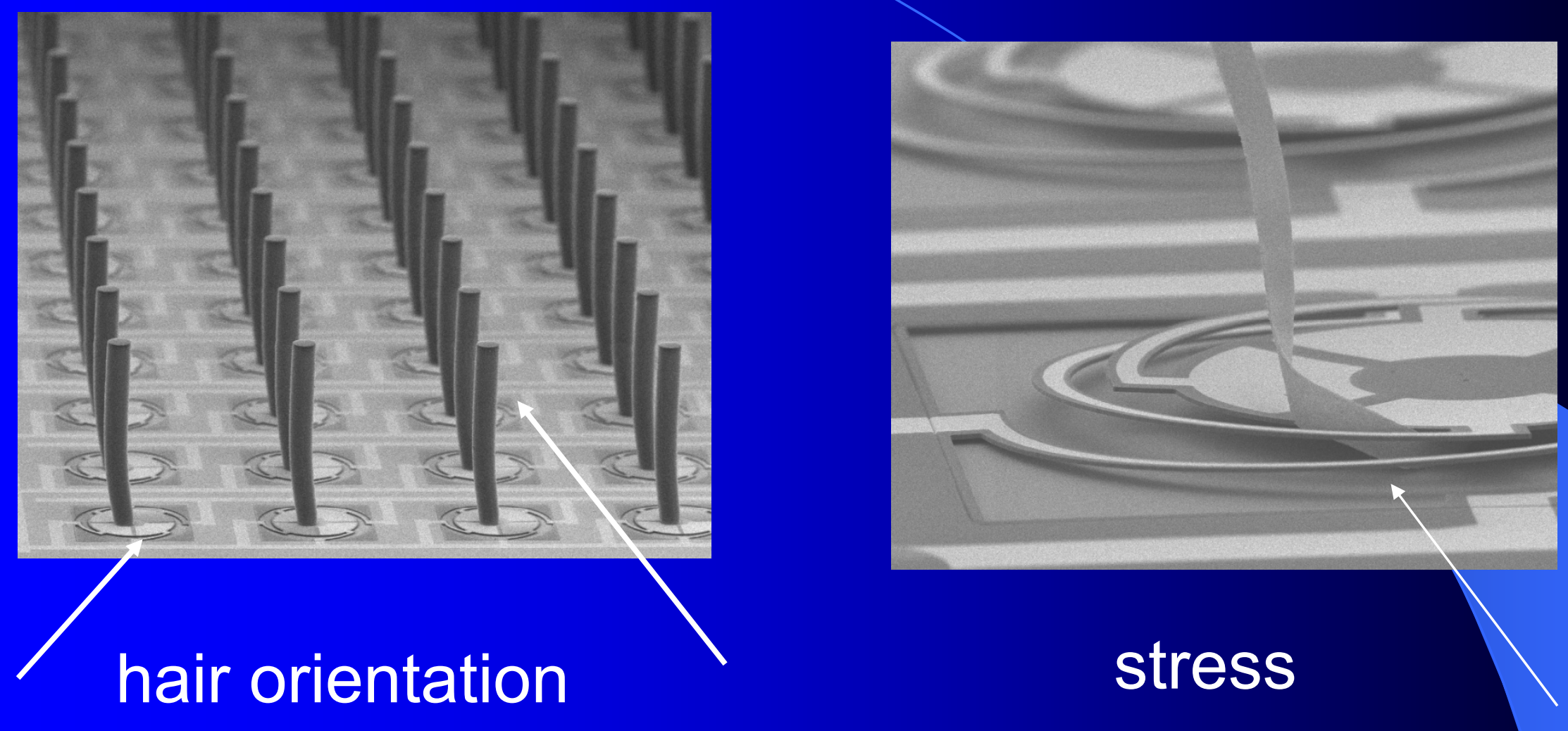


\section{MEMS Pressure Interface}

Using a pressure chamber

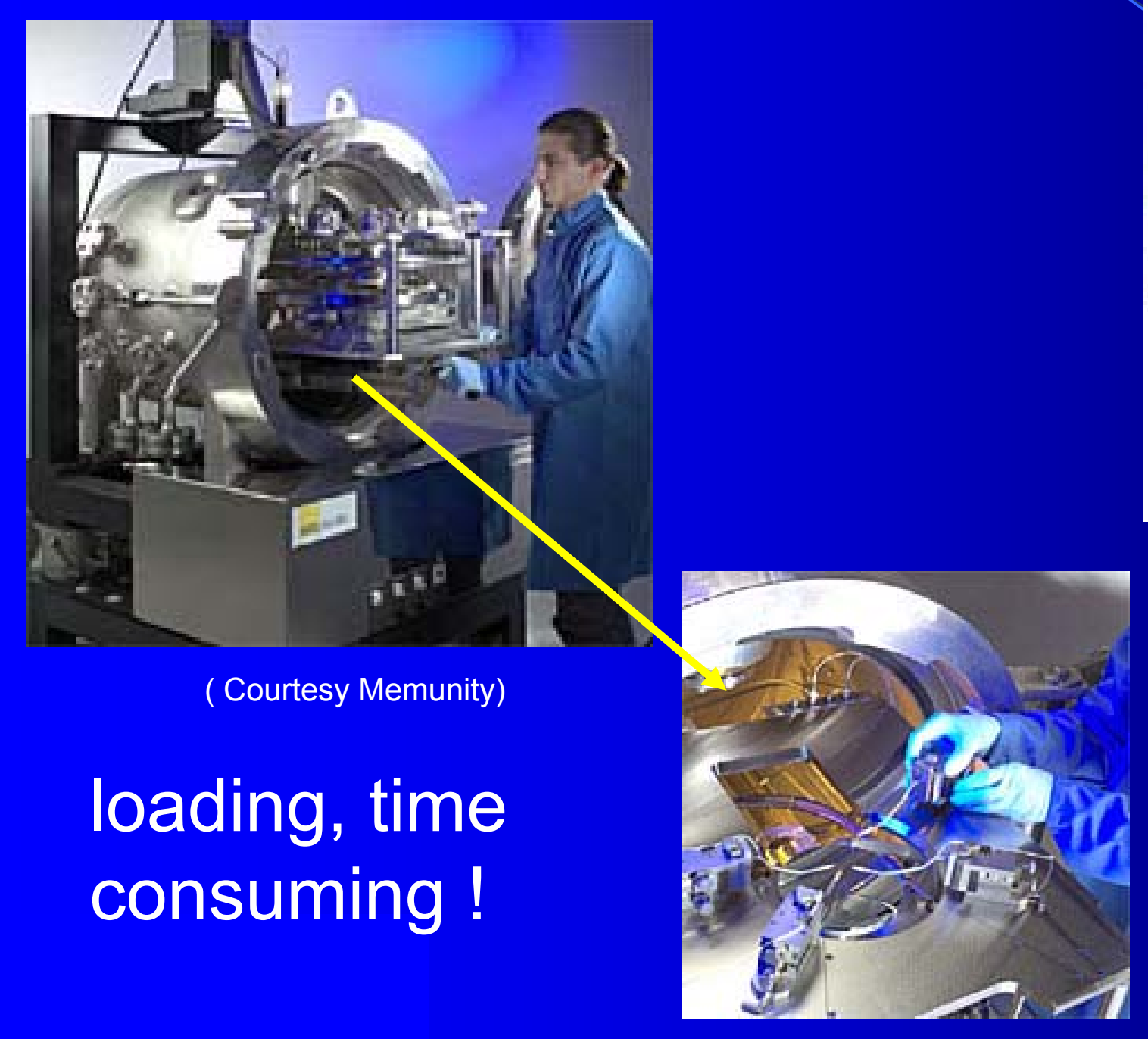

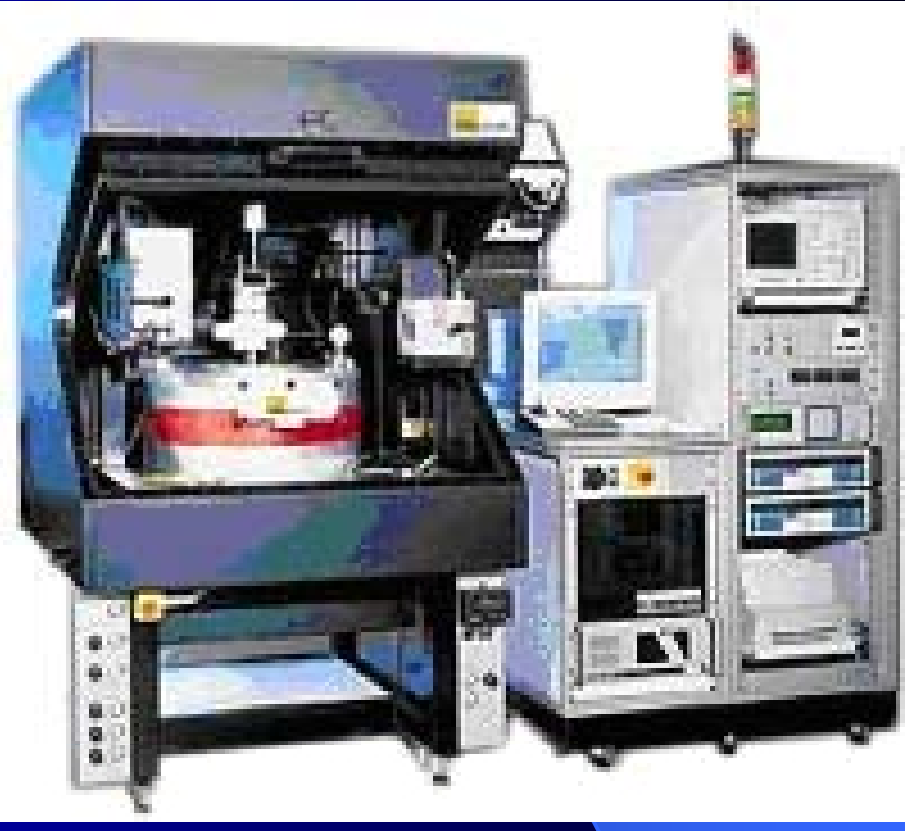

Vacuum chamber

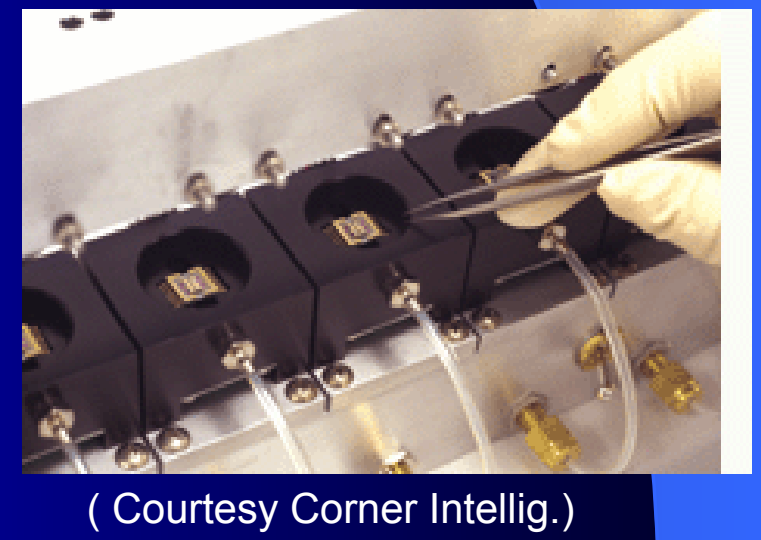

Testable Design for MEMS Sensors 


\section{MEMS Pressure Microsystem}

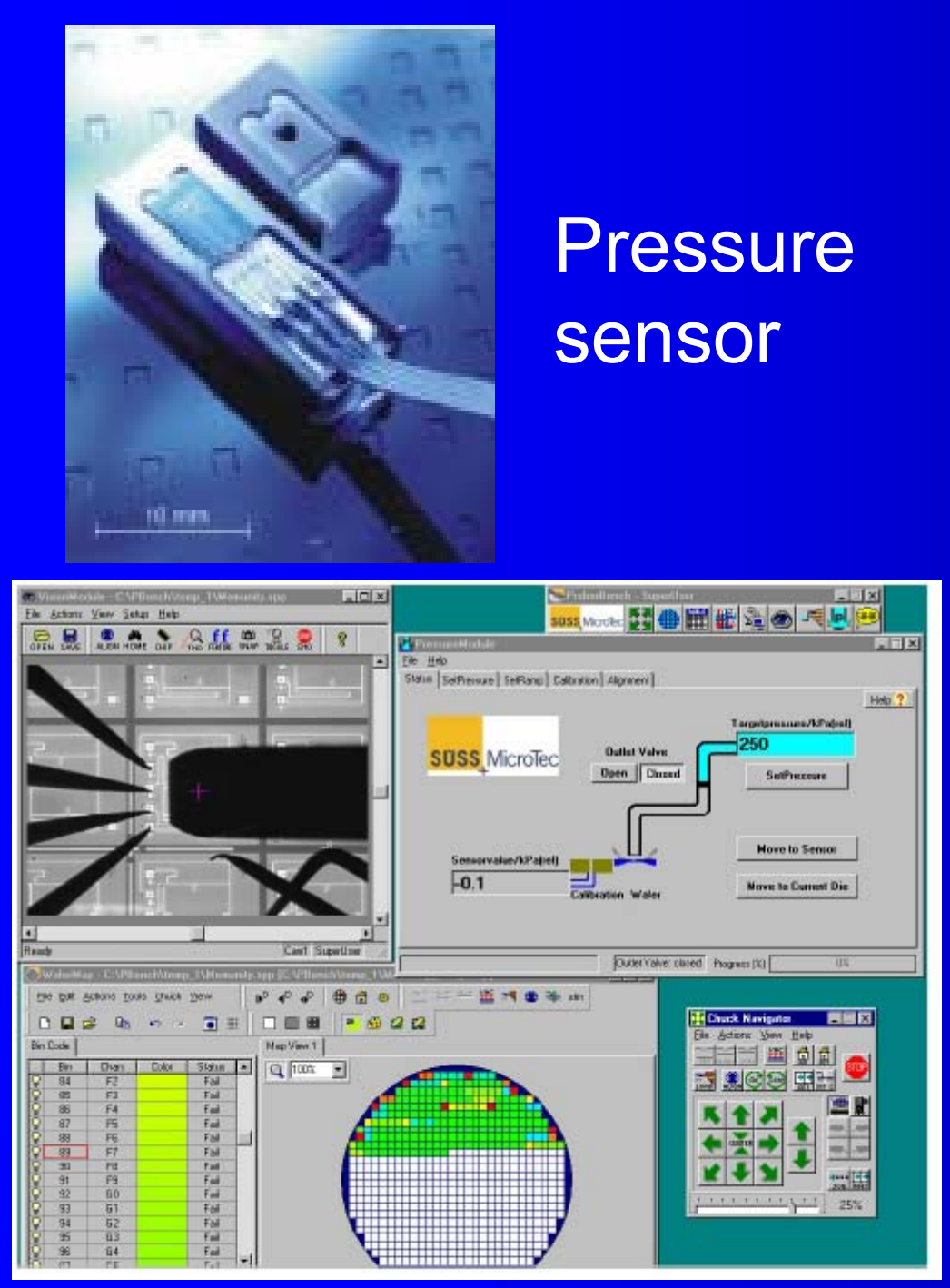

Results

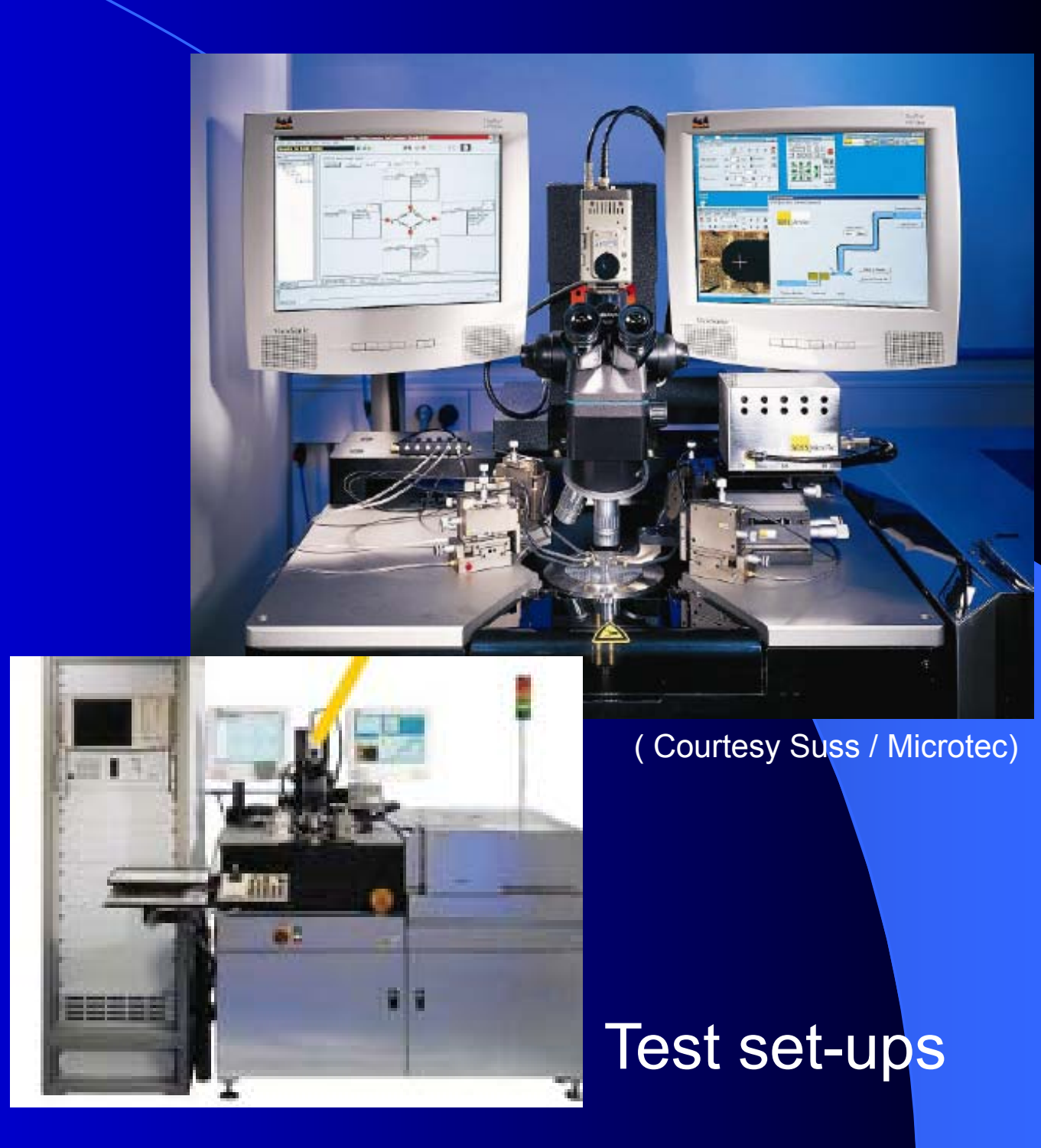

Testable Design for MEMS Sensors 


\section{Inkjet Testing \& Reliability}
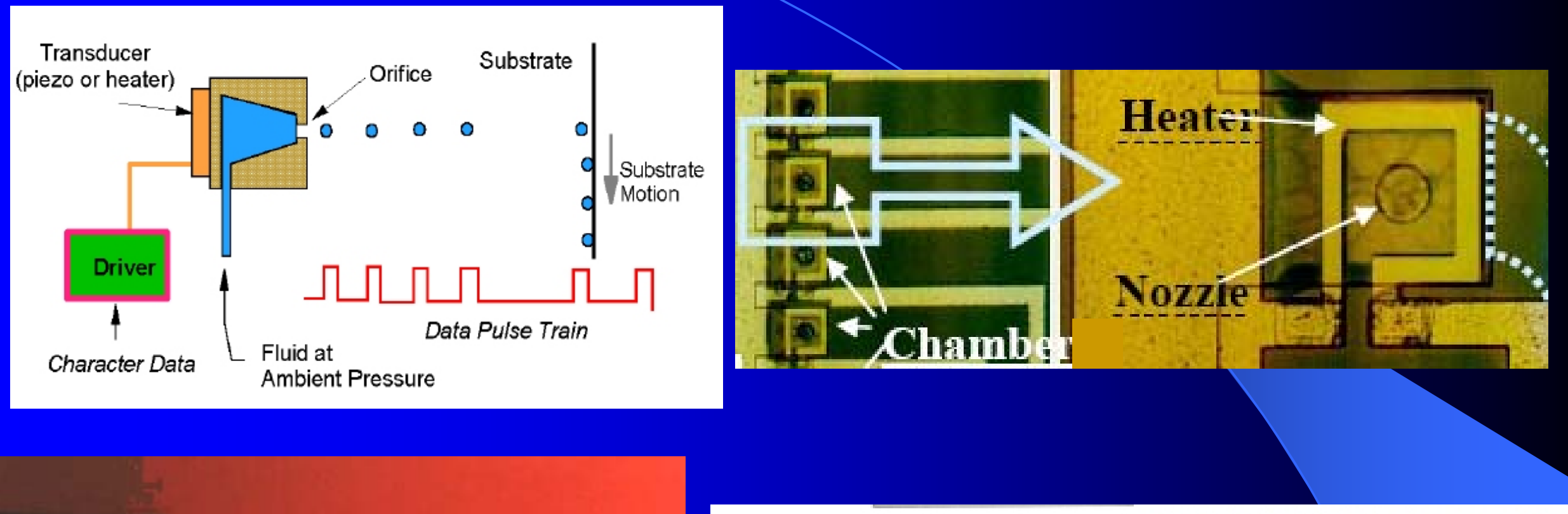

Nozzle: $60 \mu \mathrm{m}$ Droplet: $50 \mu \mathrm{m}$ Frequency: $8 \mathrm{kHz}$

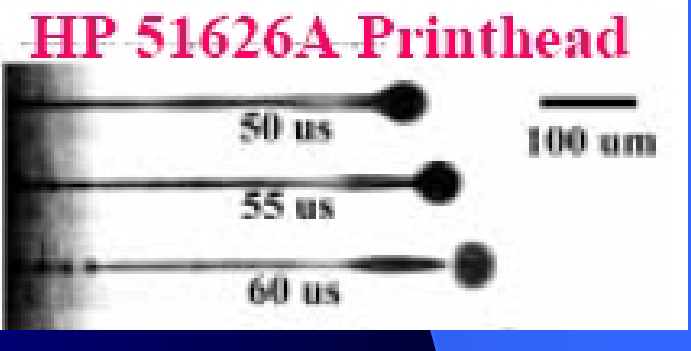

( Courtesy Imtec)

Electrical test at wafer (e.g. heater), imaging later 


\section{Inkjet MEMS \& Reliability}
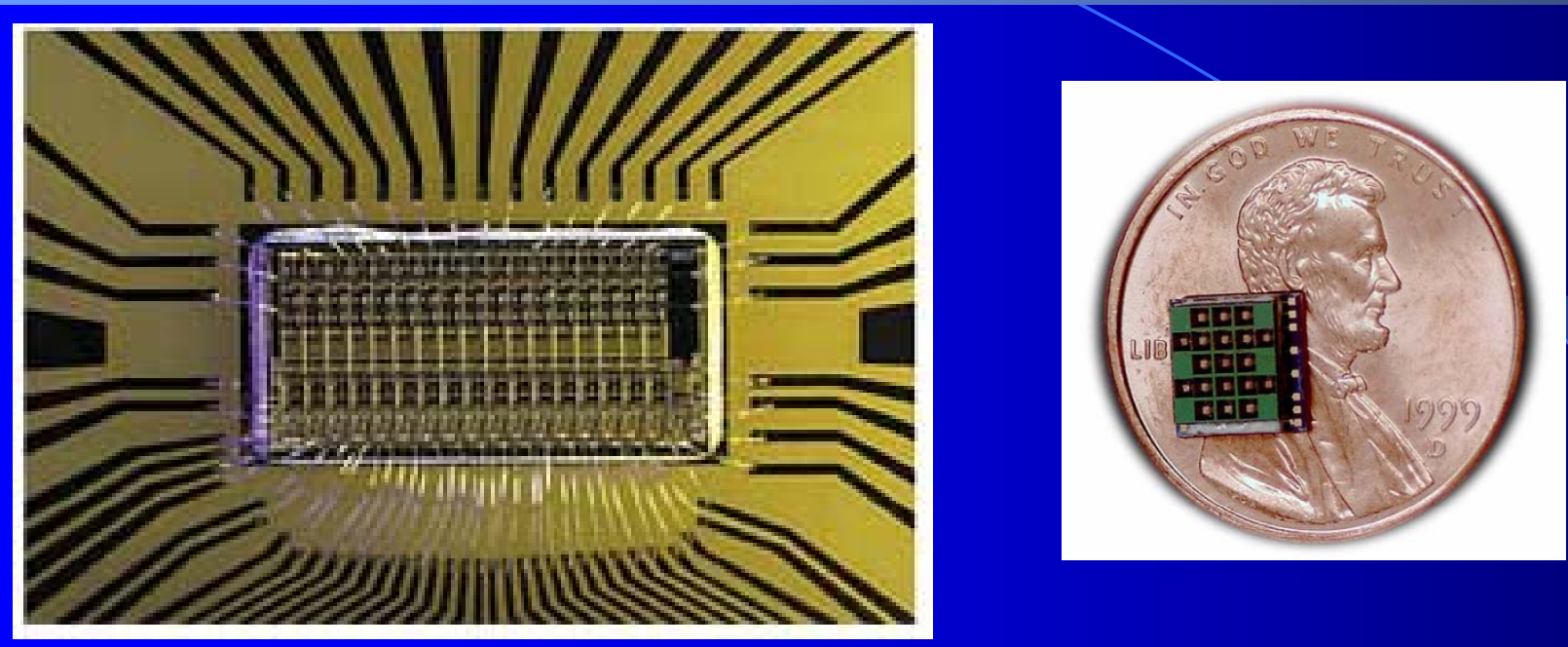

Reliability is big issue in MEMS (safety)
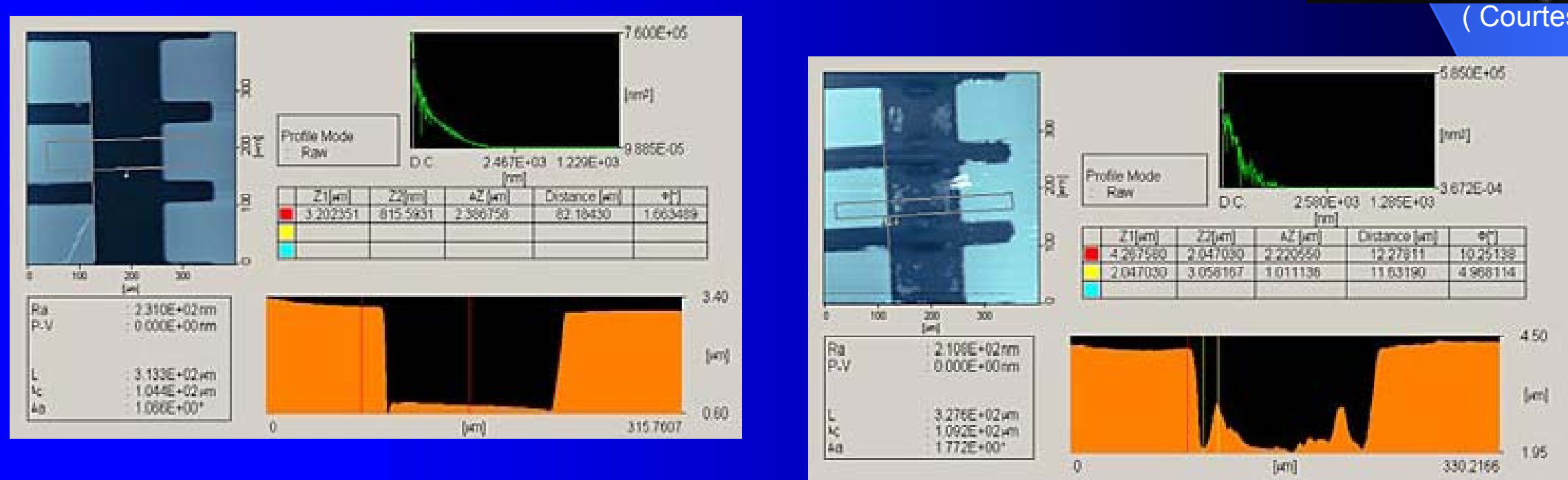


\section{Quality Metrics: Structural Test \& DfT}

8uality needs a unit of measure (like e.g. FC)

- Functional / specification-based tests are not sufficient to detect the different defects

- Like analogue electrical, domains feature masking etc.

Should detect on the basis of expected defects

2 DfT could be required to increase observability \& controllability of local points of interest

Often after front-end electronics (noise, amplitude) 


\section{Design for Test}

- First step is bidirectional domain conversion, second step is signal choice \& signal processing

- Complexity of on-chip bidirectional conversions is highly dependent of domain: basically sensor/actuator

Relative simple conversions: e.g. temperature, magnetic field, optical

- More complex conversion: gas flow, fluidic flow \& pressure 


\section{Example of DfT for Microsystem (1)}

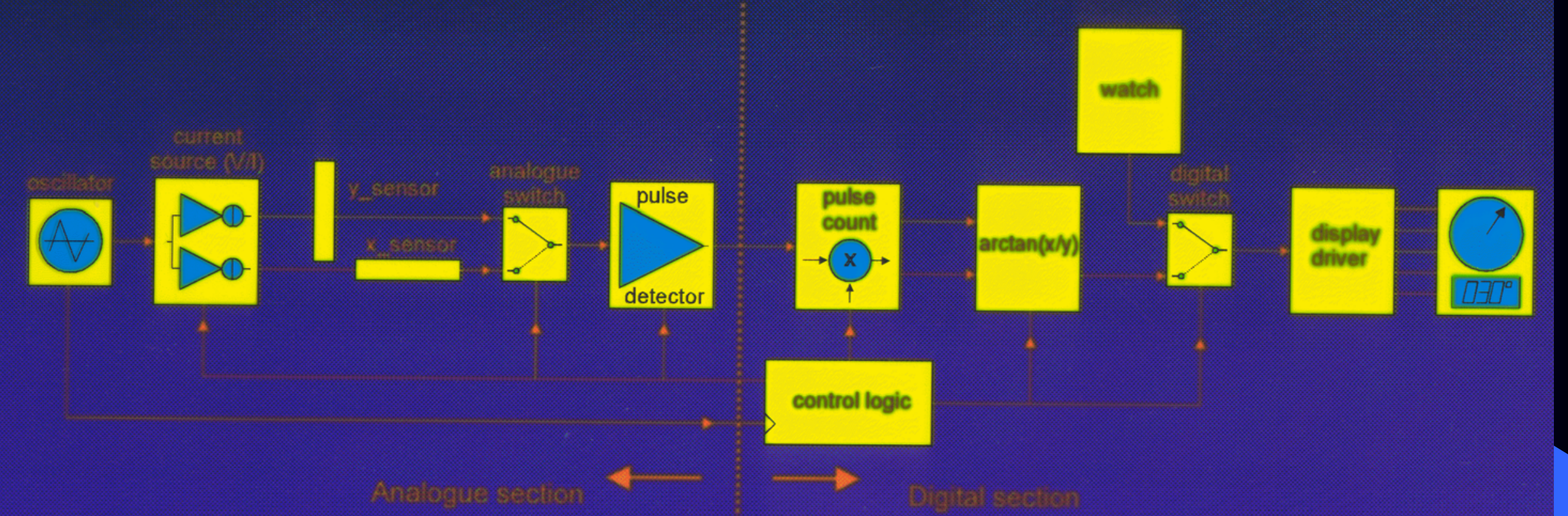

architecture of Compass Watch

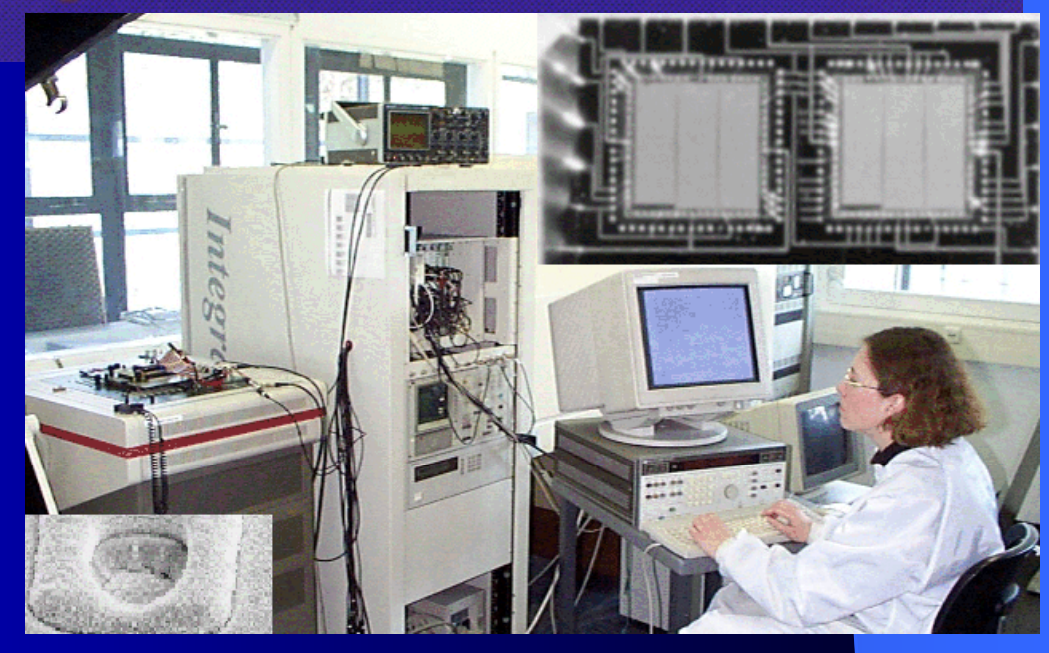




\section{Example of DfT for Microsystem (2)}

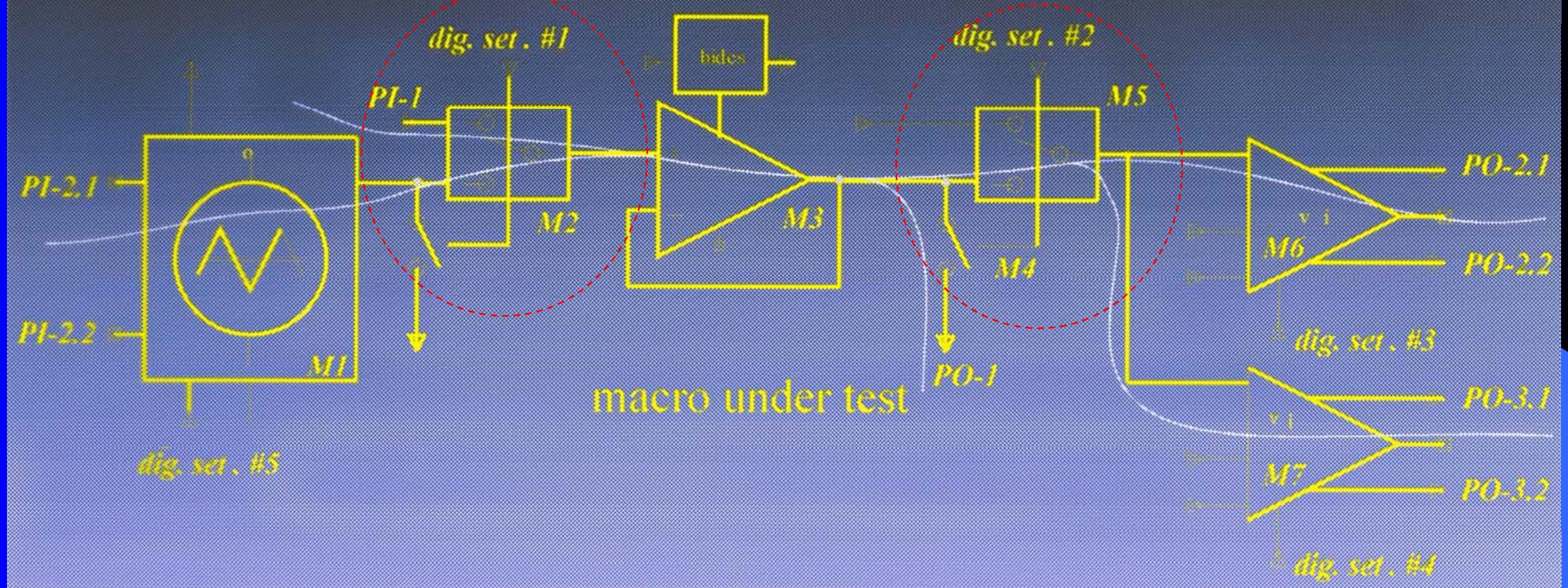

Test path of Compass Watch 


\section{Example of DfT for Microsystem (3)}
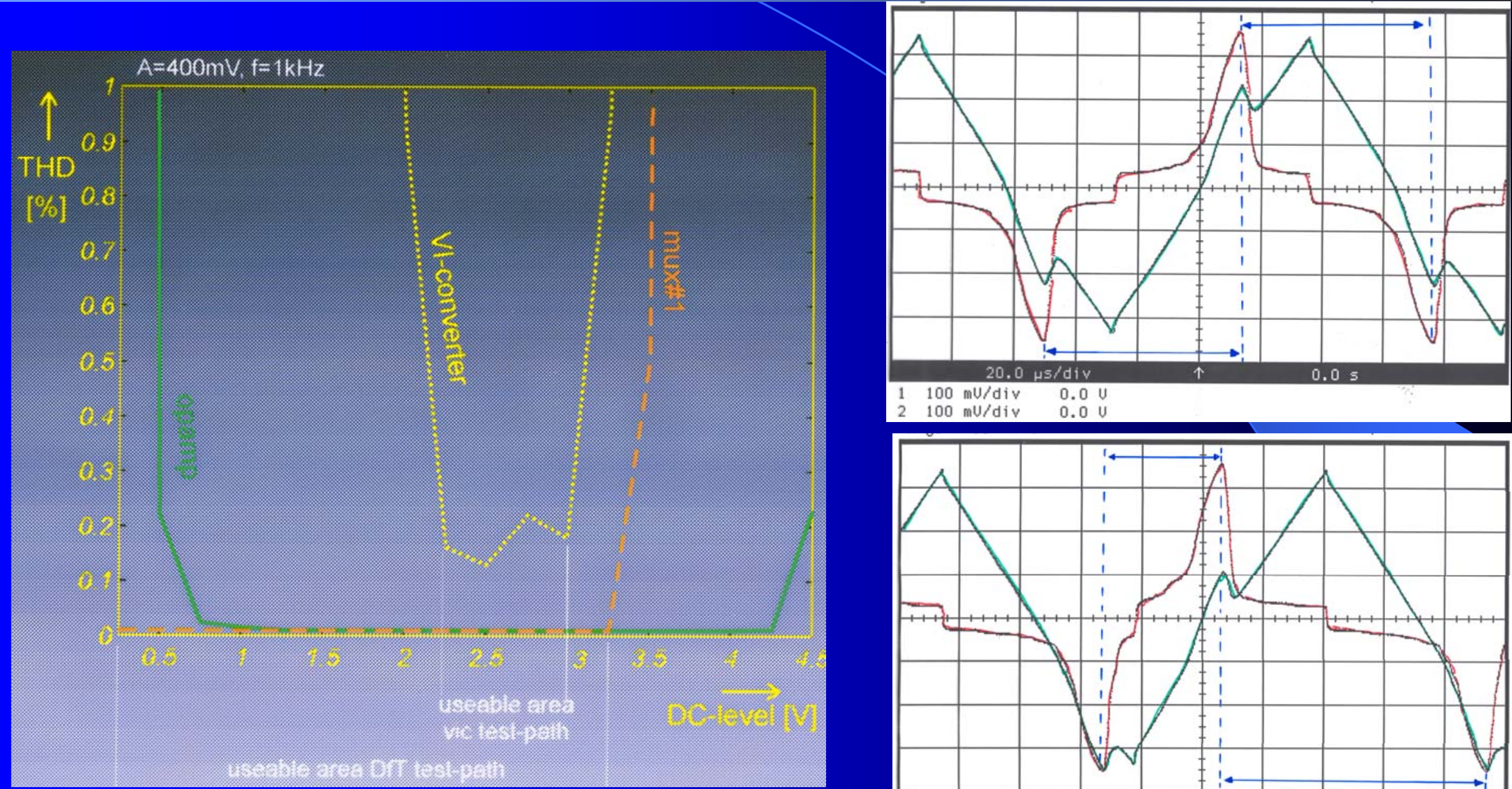

$100 \mathrm{mU} / \mathrm{div}$
$100 \mathrm{mU} / \mathrm{di}$

$0.0 \mathrm{U}$

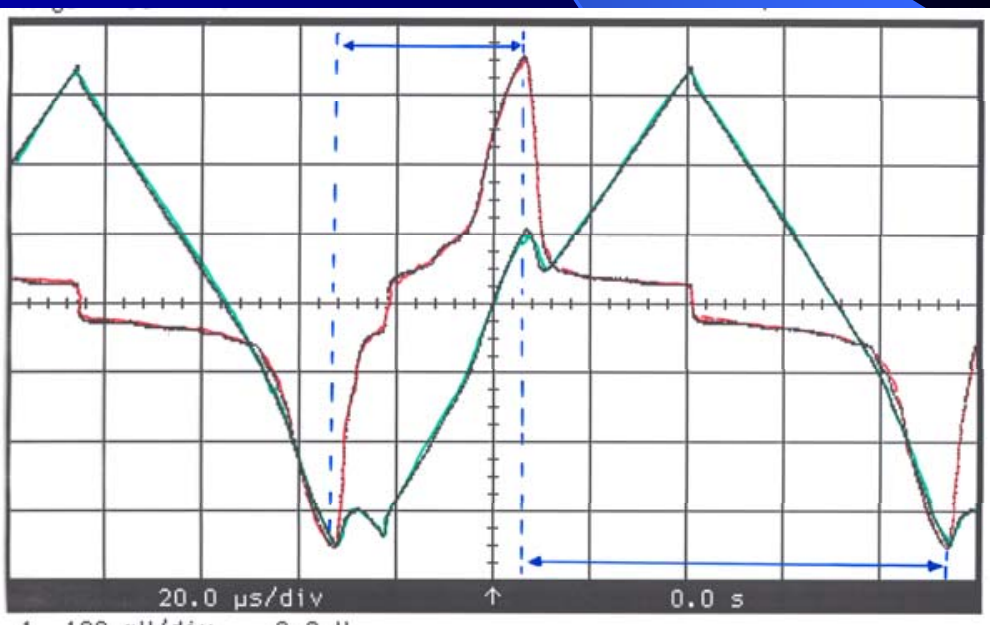

Check on multiplex \& measure 


\section{Stimulation \& Observation of MEMS}

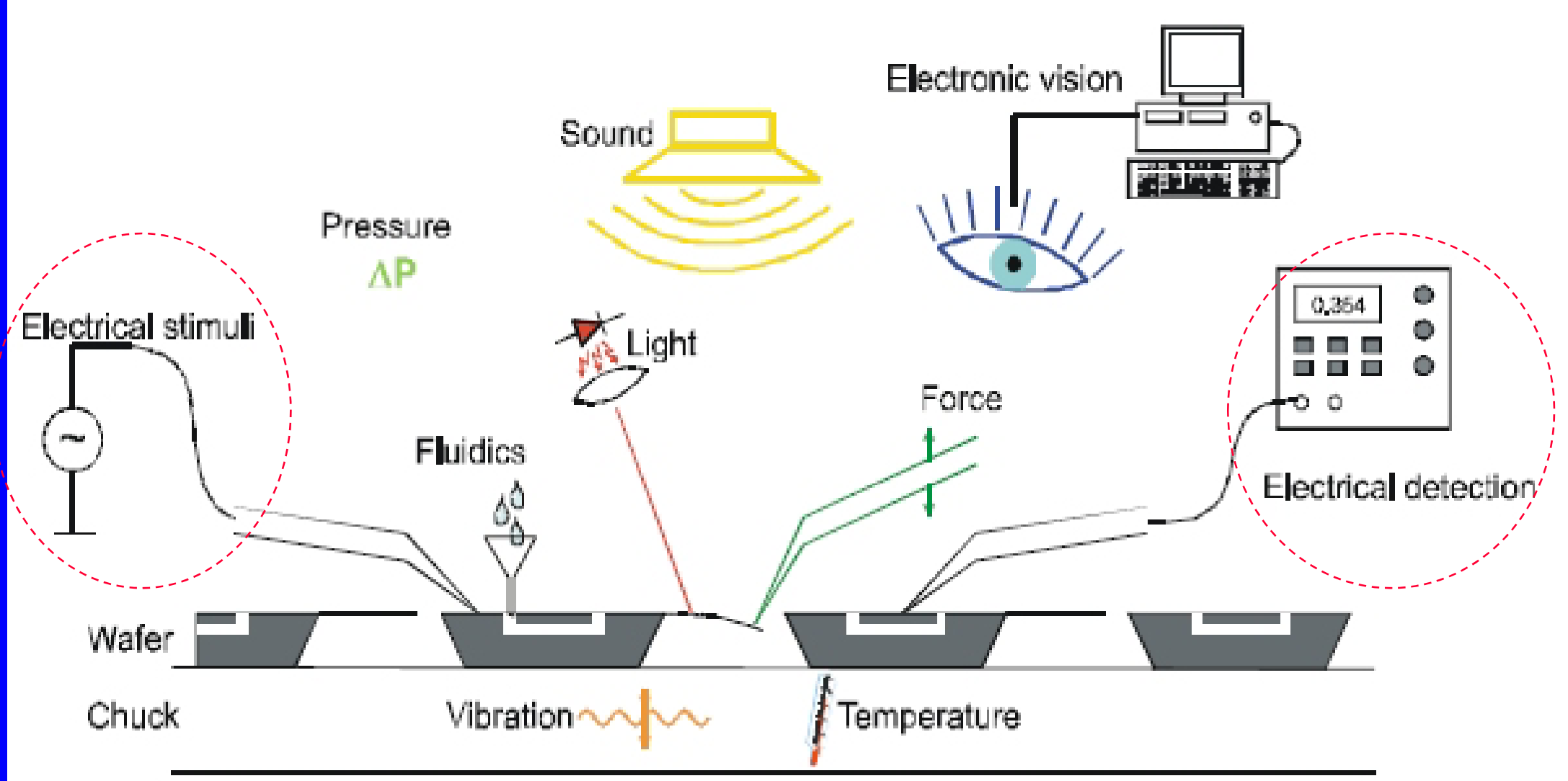

Cumbersome interfaces and often slow 


\section{Generic Design for Test Approach}

\section{Domain $\mathrm{A}$}

Domain B

e.g. resistor, photo transistor

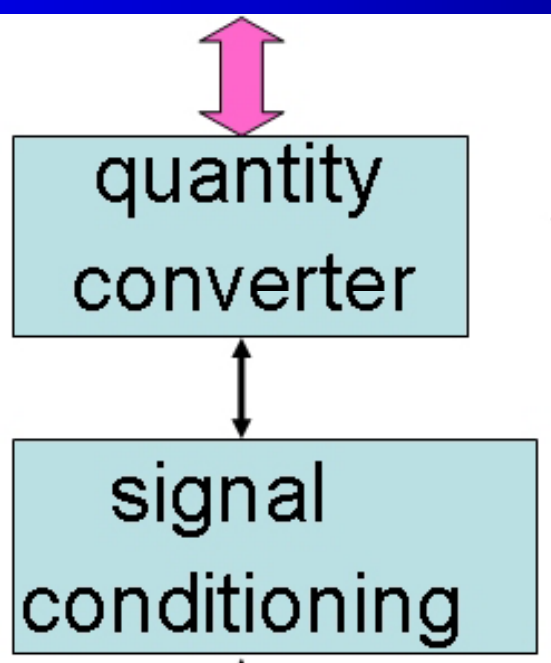

e.g. opamp, filter, dataconv conditioning

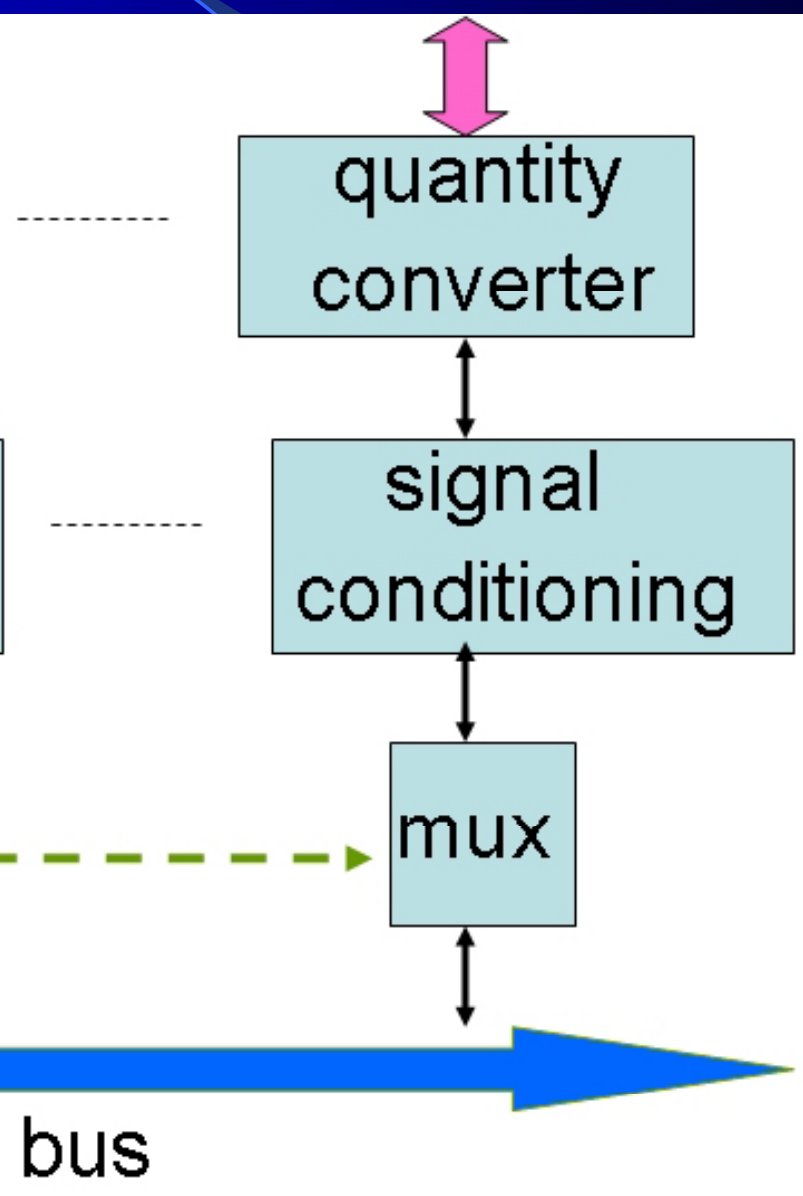

e.g. digital scan chain

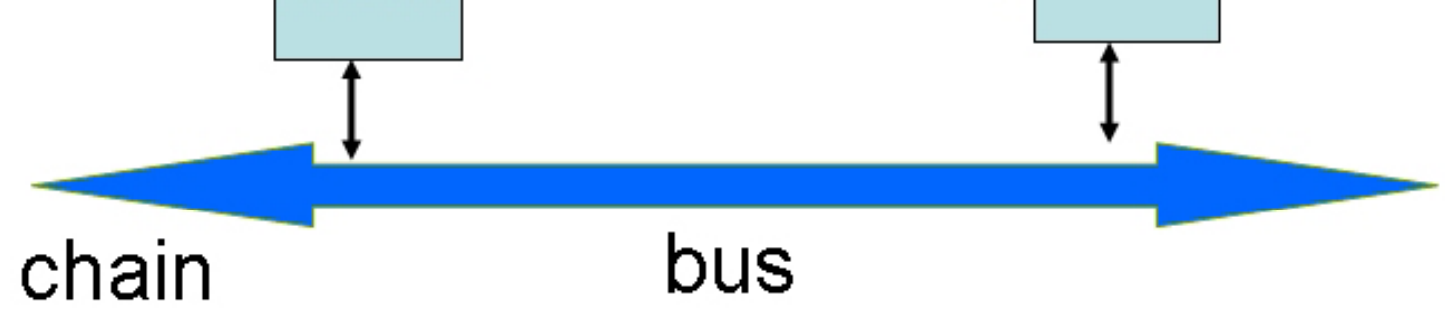




\section{Possible DFT Interfaces for MEMS}

\begin{tabular}{|l|l|l|c|}
\hline Domain & Stimulus device & Observe device & complexity \\
\hline Temperature & Heater resistor & Temp resistor & ++ \\
\hline Magnetic B & Coil & Coil / B sensor & + \\
\hline Light & Photo diode & Photo transistor & -- \\
\hline Pressure & Heat membrane & Piezo resistor & - \\
\hline Movement & Comb structure & Capacitor / piezo & -- \\
\hline Fluidic flow & FlowFET & Heat / sense R/C & - \\
\hline
\end{tabular}




\section{Some DfT Components for MEMS}

heater \& piezo R

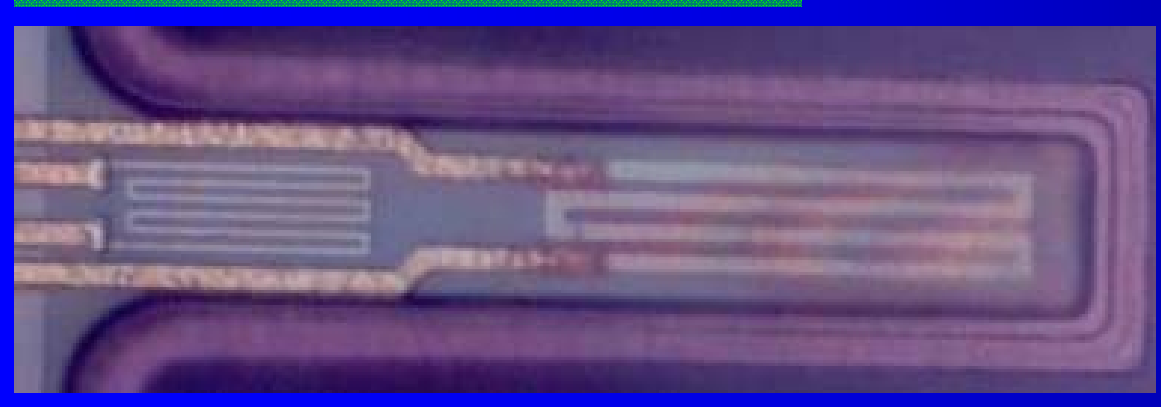

( Courtesy TIMA)

\section{Actuation \& sense comb}
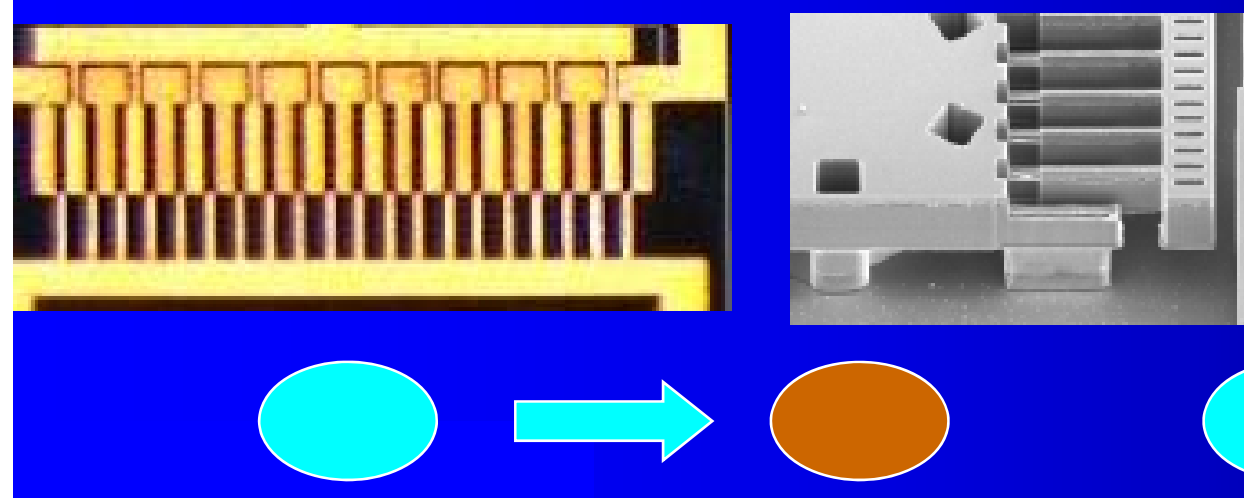

\section{stim \& pickup coil}

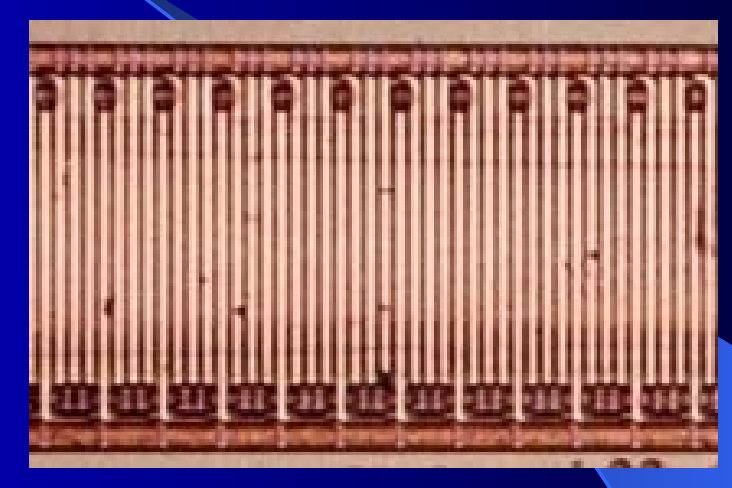

FlowFET, Flowsensor

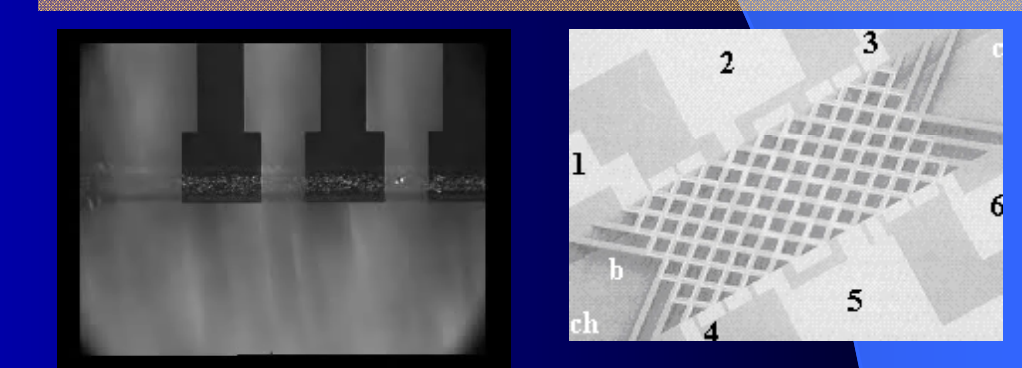




\section{Typical Fluidic Test Setup}

Injection

- Valves

- Slow

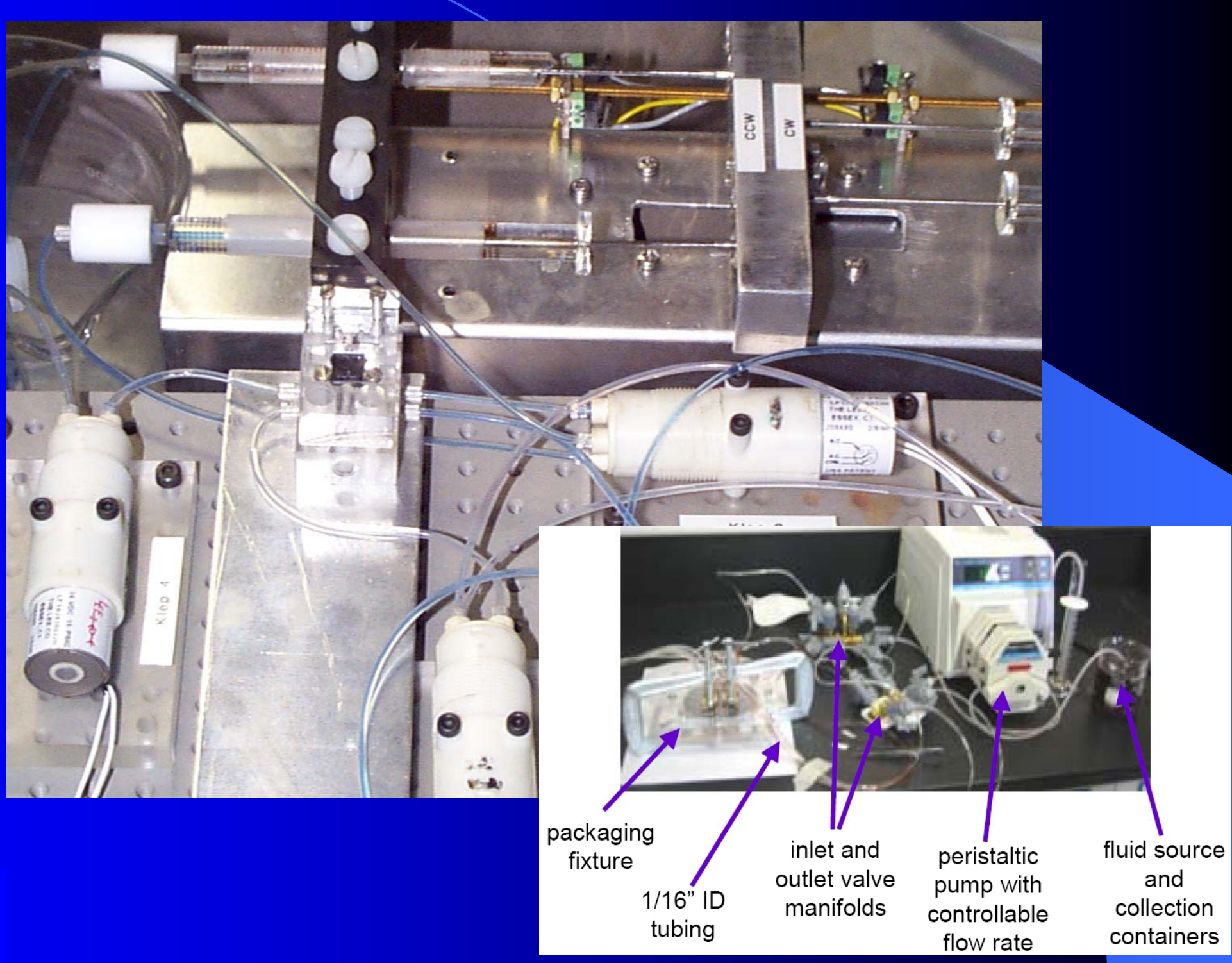

Testable Design for MEMS Sensors 


\section{Testing Fluidic Flowsensor \& Pump}

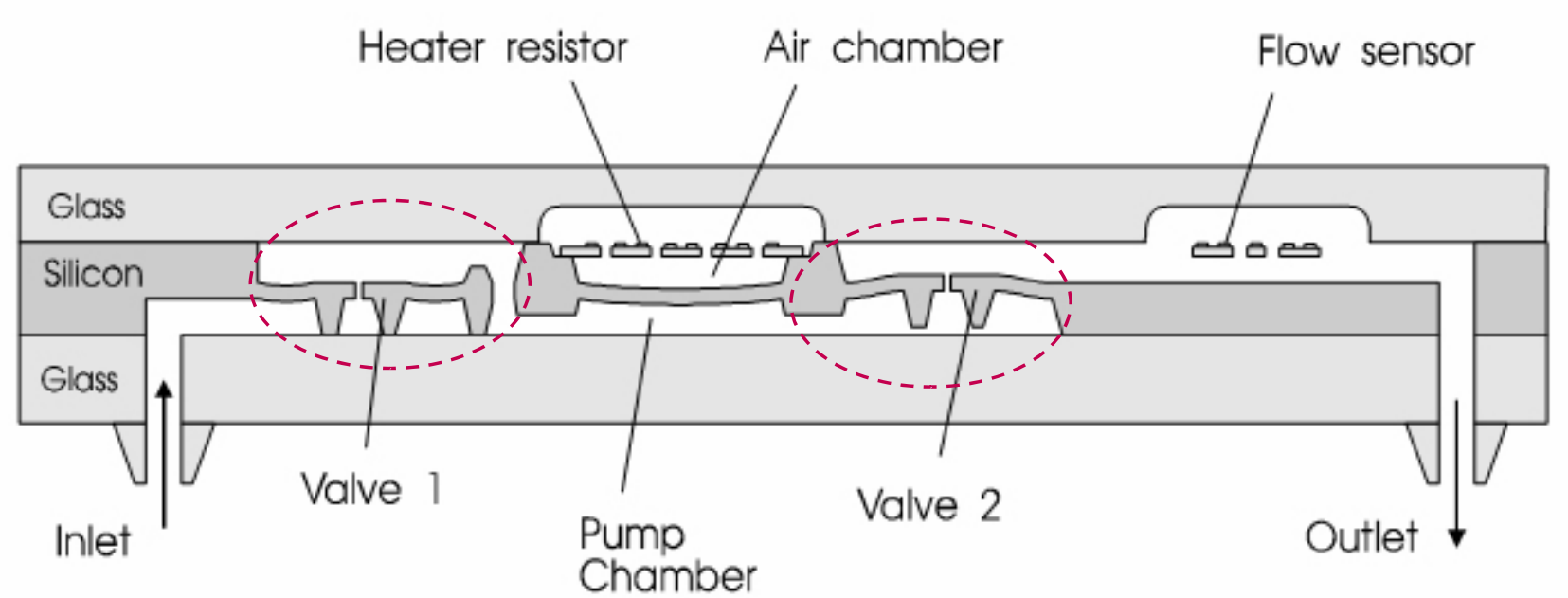

Mechanics creates problems

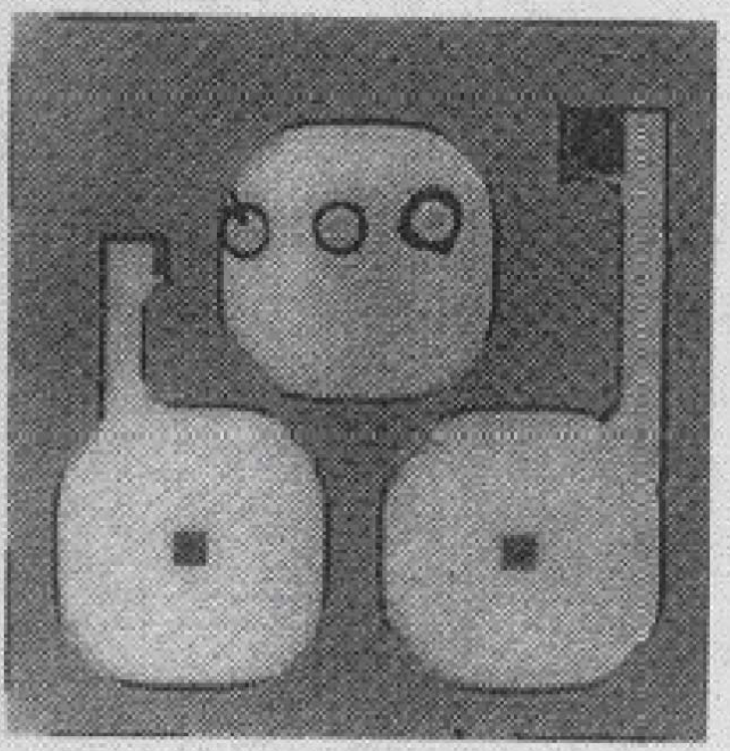

- DfT interfaces: - capacitive, switch (metal layers)

- piezoresistive 


\section{Electrical DfT in MAS System}

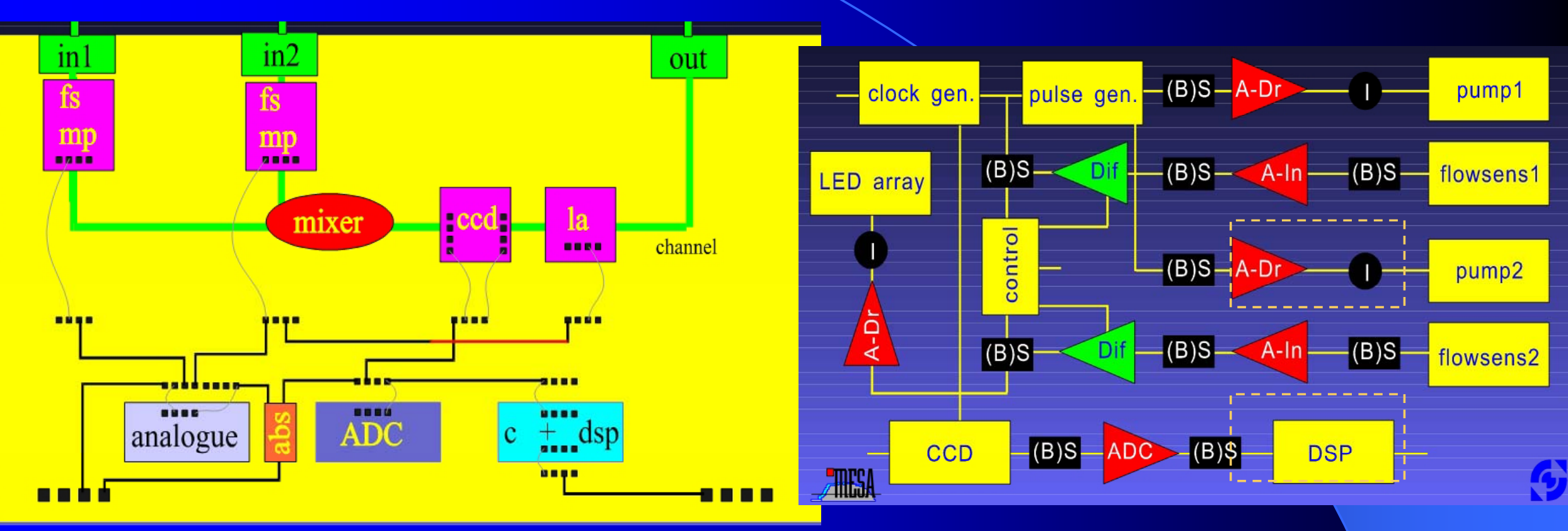

\section{Basic scheme}

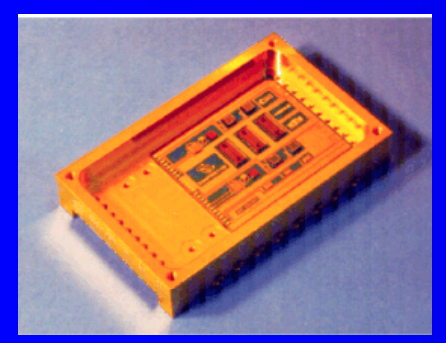

( Courtesy Twente Micro Products \& HP)

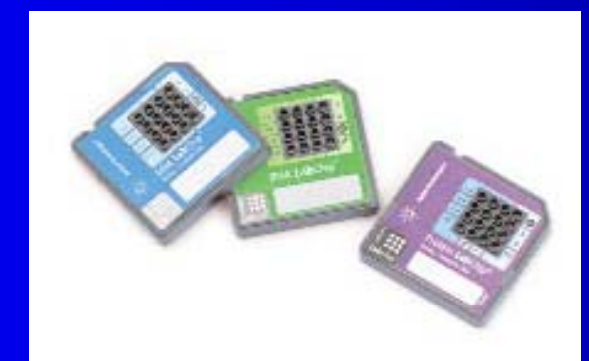

\section{DfT MAS}

Use electrical wafer pre-tests before packaged fluidic functional tests

\section{implementations}




\section{Architecture Analogue MEF Array}

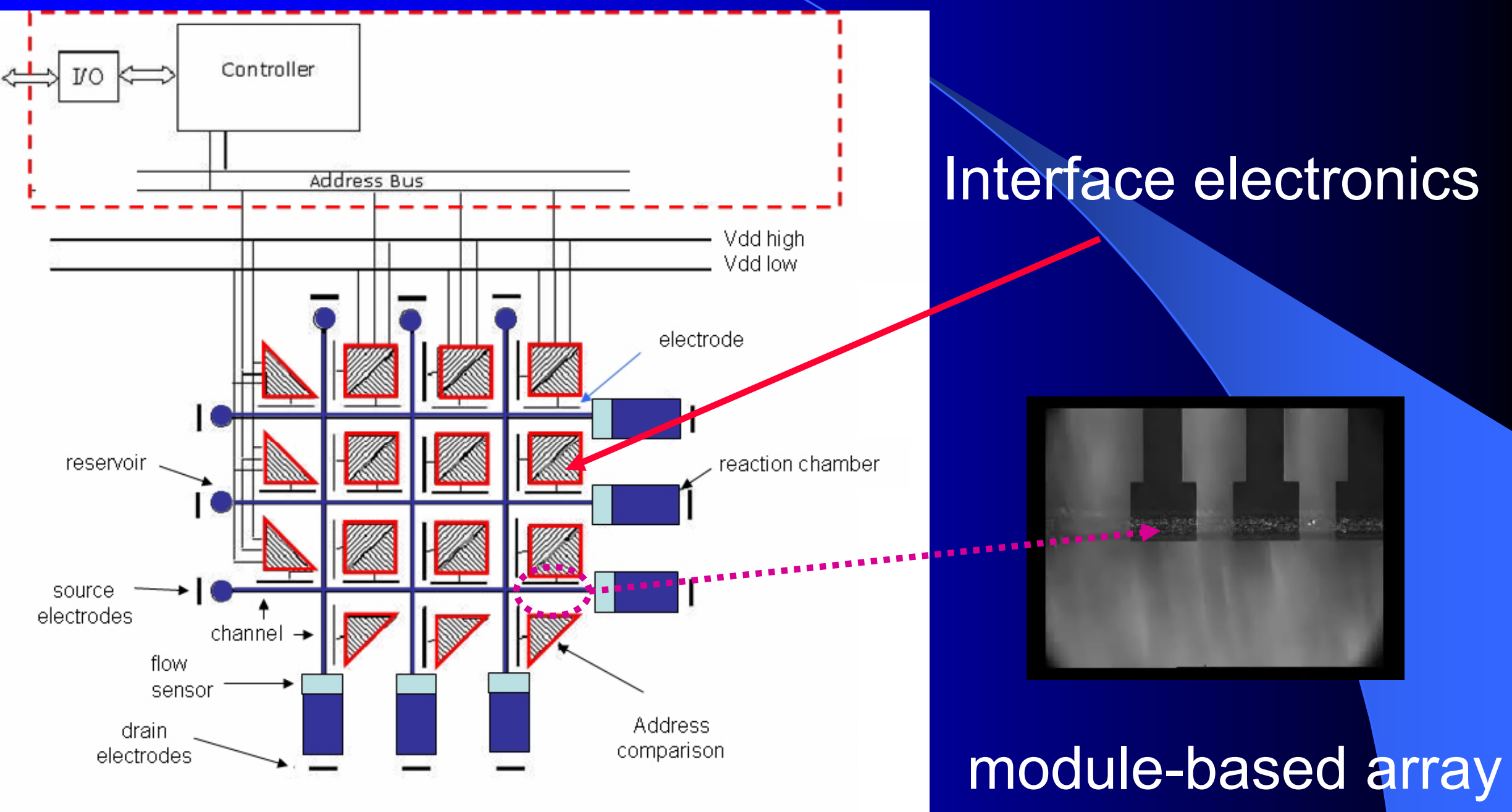




\section{Testing Interface Electronics}

Use Iddq monitoring
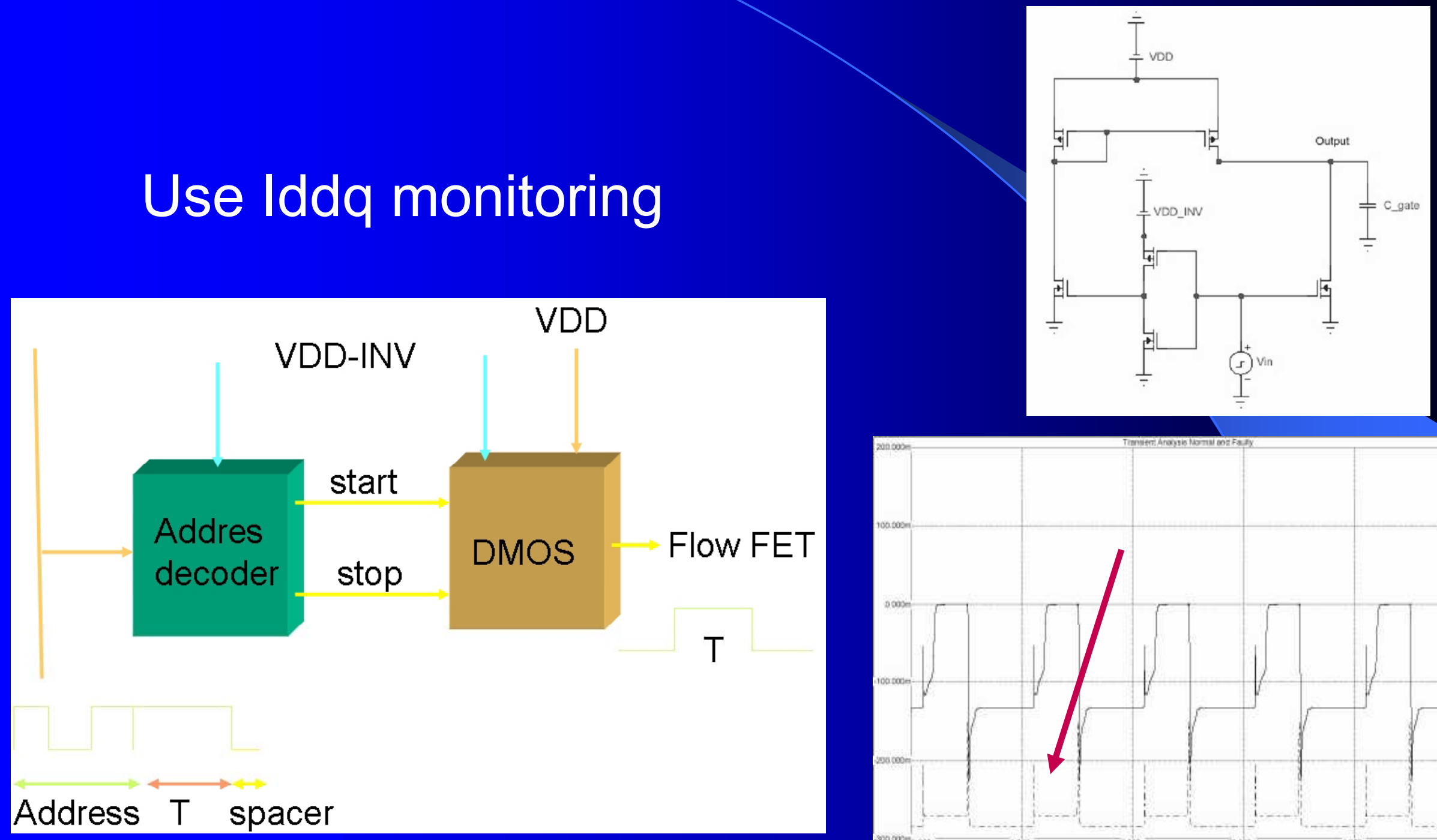

Address $\mathrm{T}$ spacer

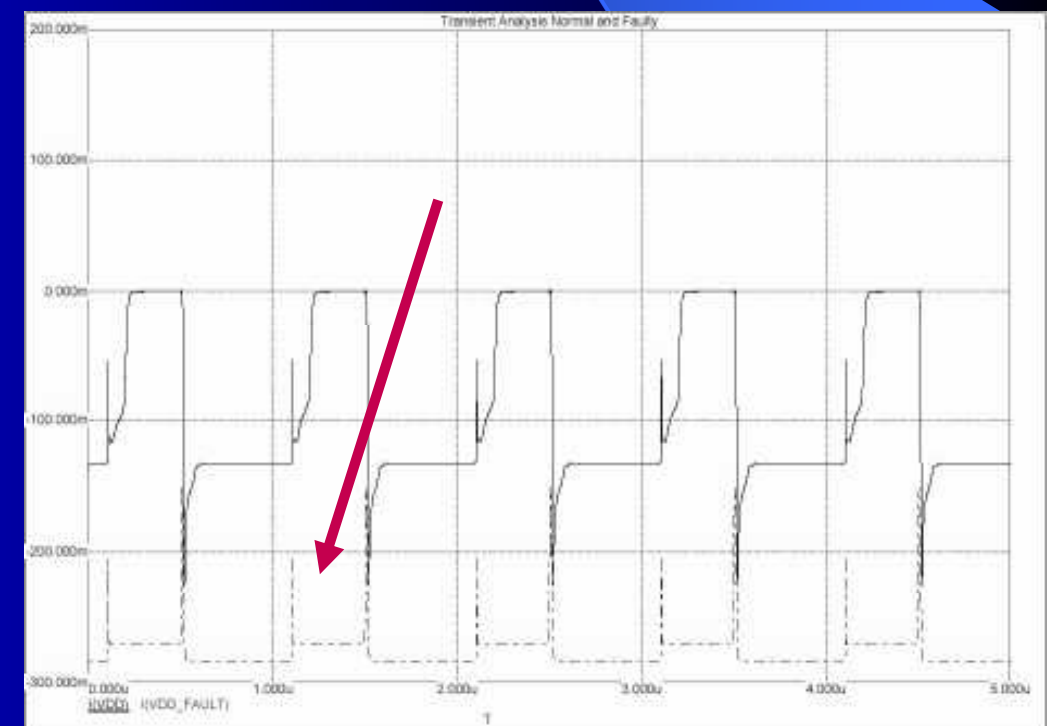




\section{High-level Multi-domain Simulations

MathLab , VHDL-AMS are being used in multi-domain

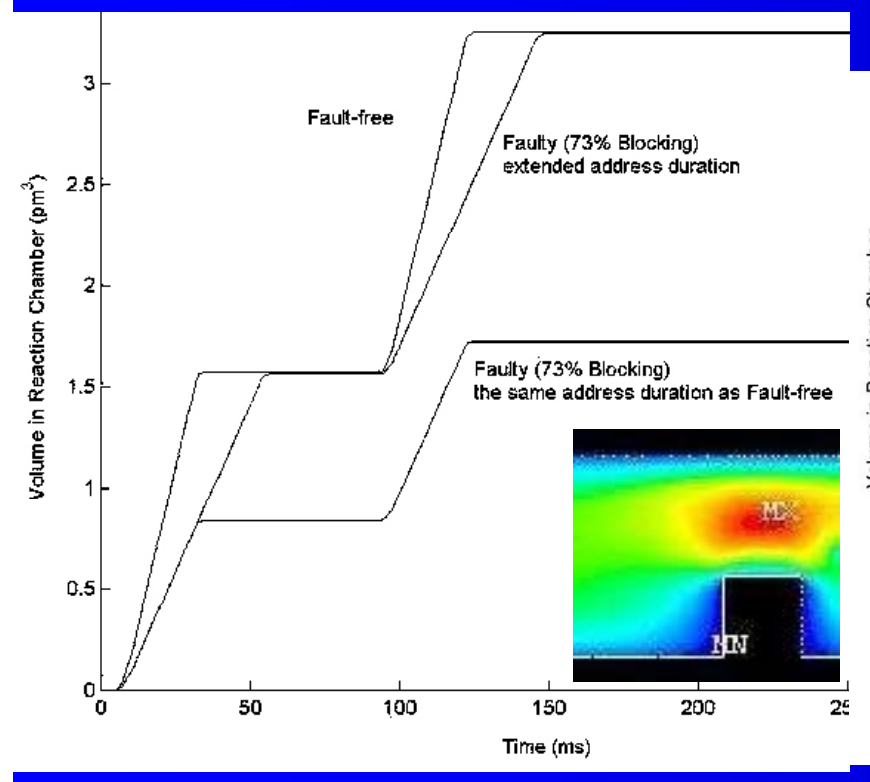

particle jamming

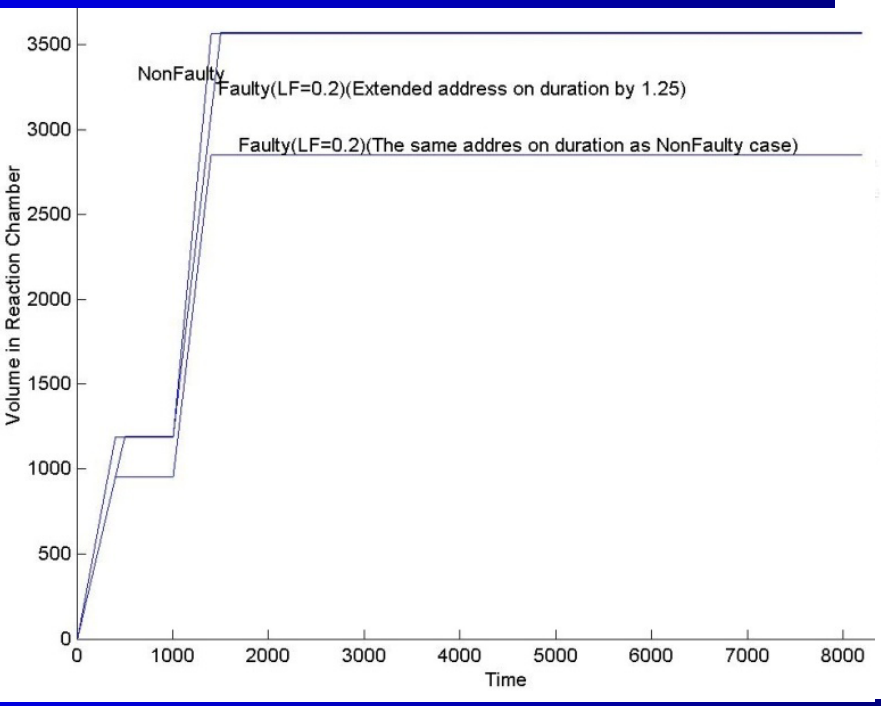

leaking

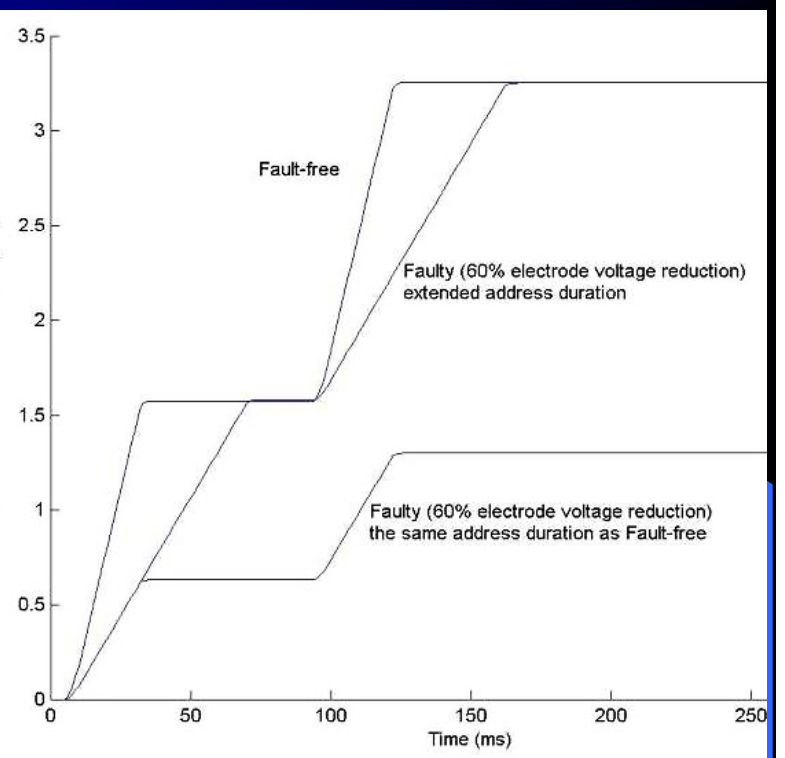

addressing

System simulations reveal possible test approach! Like in analogue parameters, speed and magnitude 


\section{VHDL-AMS Fault Simulations (1)}

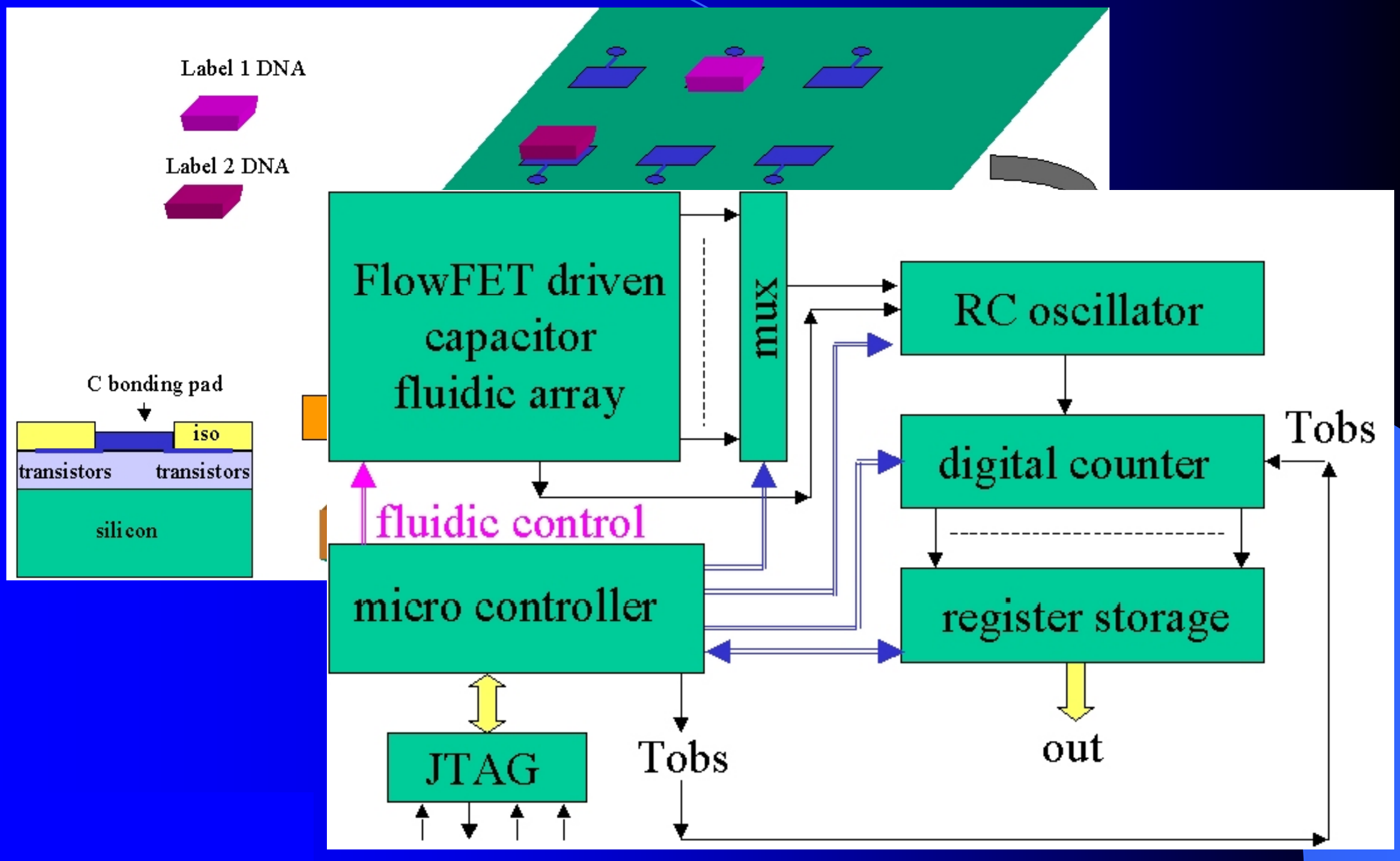

Testable Design for MEMS Sensors 


\section{VHDL-AMS Fault Simulations (2)}

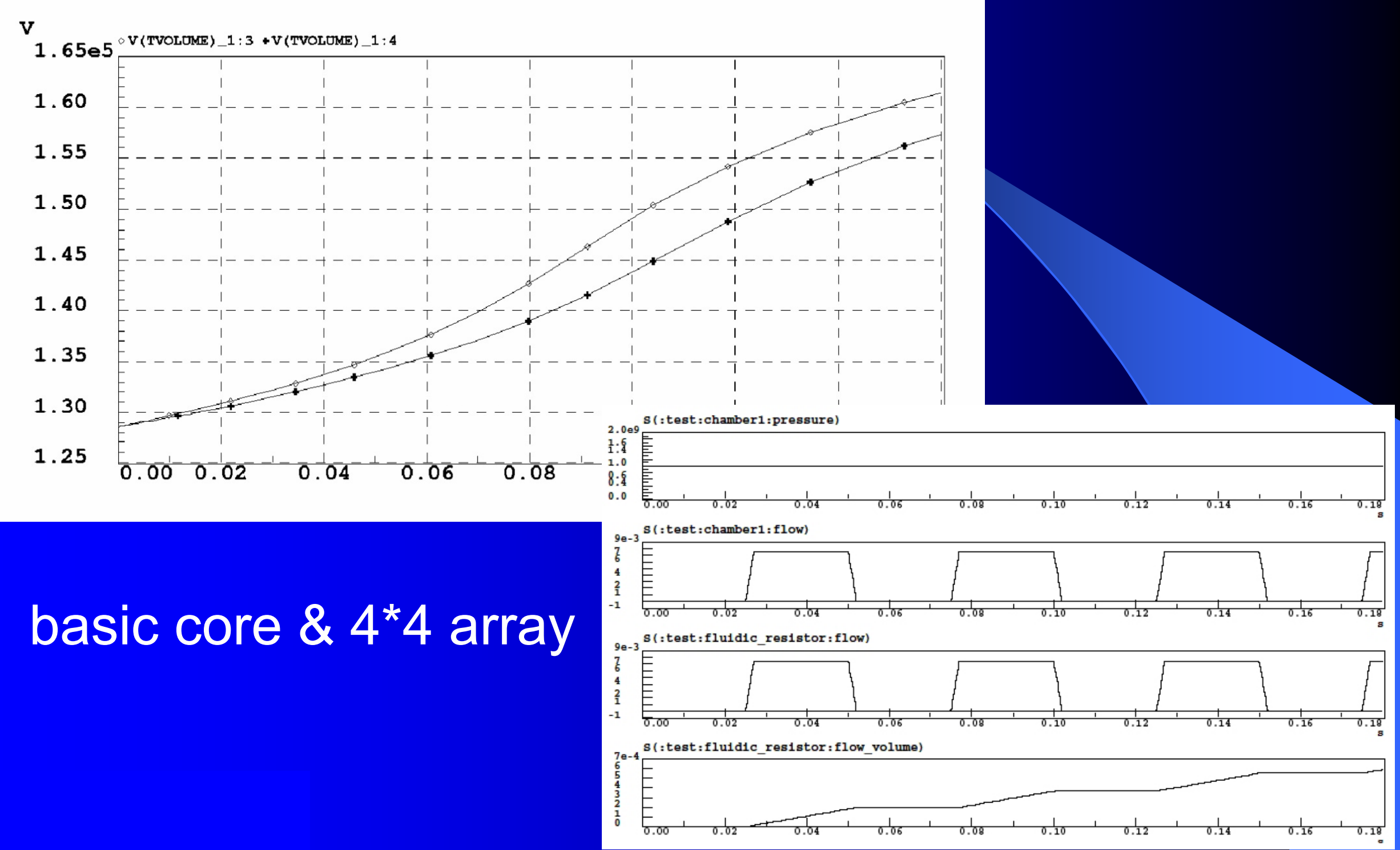




\section{VHDL-AMS Fault Simulations (3)}

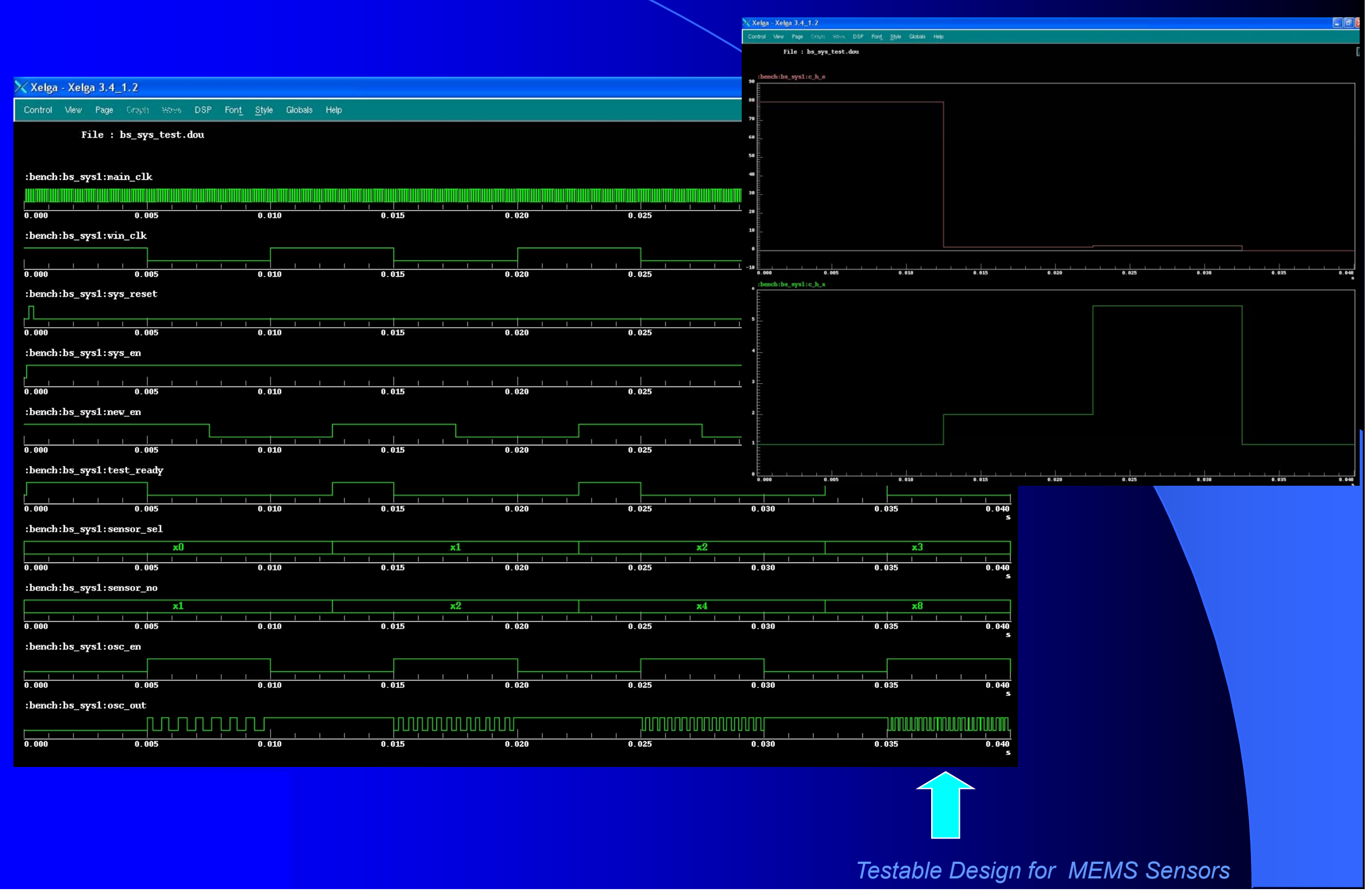




\section{MEMS Built-In Self-Testing (BIST)}

- Generate actuation stimuli locally

- Heating resistors

- Electrostatic force

- Magnetic

- Piezoresistive resistors

+ control electronics

+ signal processing

- Sensing / observation locally + comparison

- Capacitive sensors

- Electrostatic force

- Magnetic

- Piezoresistive resistors 


\section{Some MEMS BIST Approaches}

6

On-line testing via direct / indirect mixing of the test-signal with original signal

- e.g. via modulation or DC superposition (e.g. Azais, Richardson, ETS05)

Off-line testing via oscillation method

- feedback of output to input, via network (Nouet, VTS02)

$\theta$ Off-line testing via MLS application and subsequent on-chip HW correlation

- IR sensor array ( Mir, ETS05)

Differential V/g actuation \& measurement evaluation

- accelerometer 


\section{Possible BIST Approach}

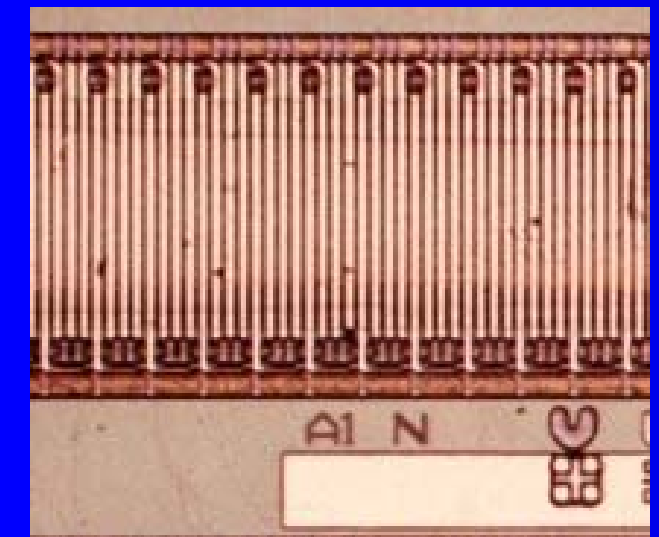

ตาว ${ }^{\prime}{ }^{-1}$

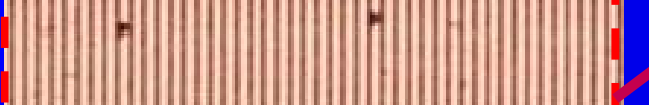

- 1 | | | | | | |

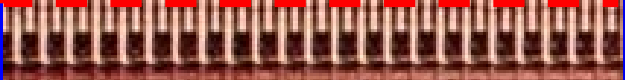

- Magnetic stimuli relative easy made

- Check coil (e.g. R)

- Check fluxgates

-coil
Alu 1

Alu 2

NiFeln

Polyimide

\section{Si and SiO2 toplayer}

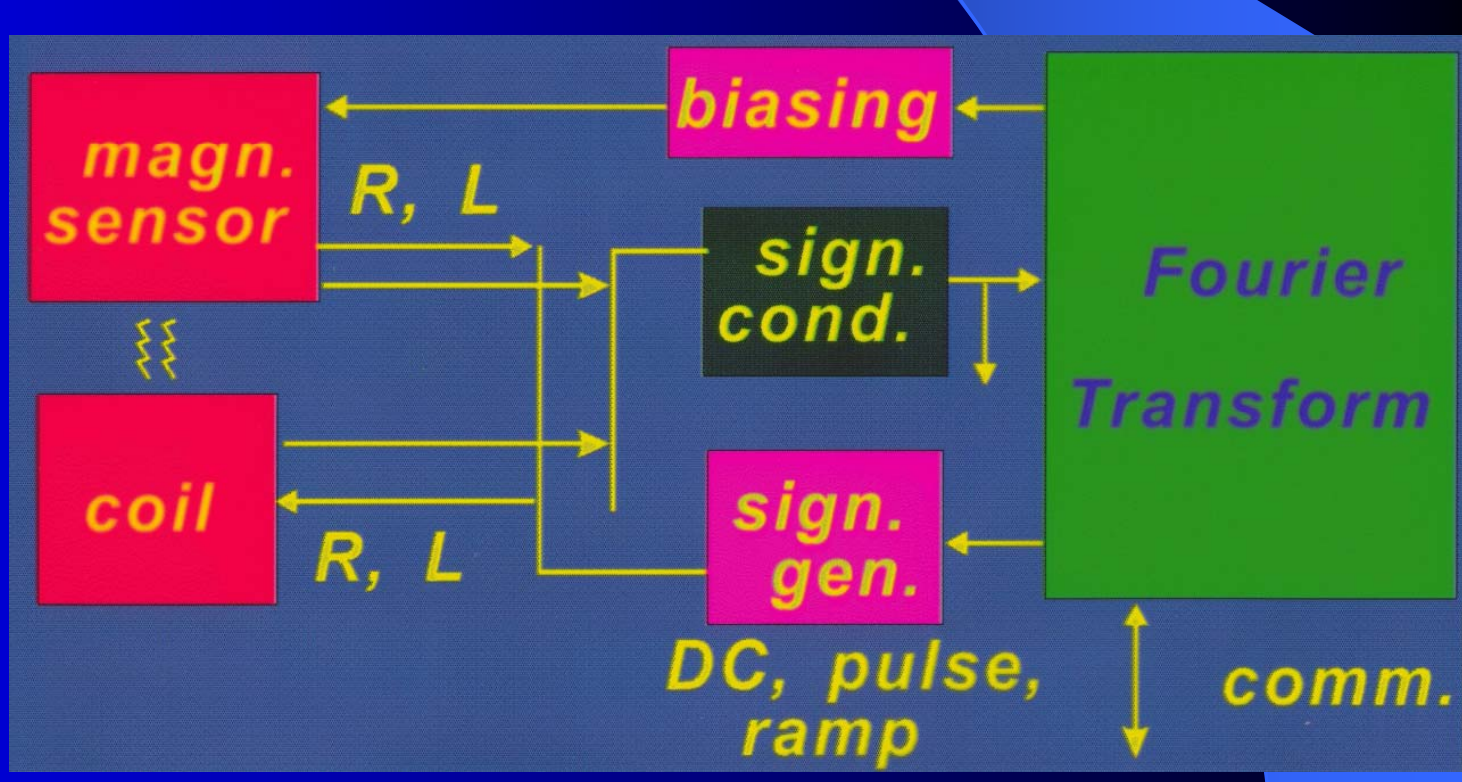




\section{Possible BIST Approach: IR Imager}

- Uses heat absorbtion and Seebeck effect

- Choose cell by $x, y$, and via test, Vtest the heater is activated

- Output is available via thermopile +,-

- Signal processing is off-chip and pure functional

- A transient input signal is being used

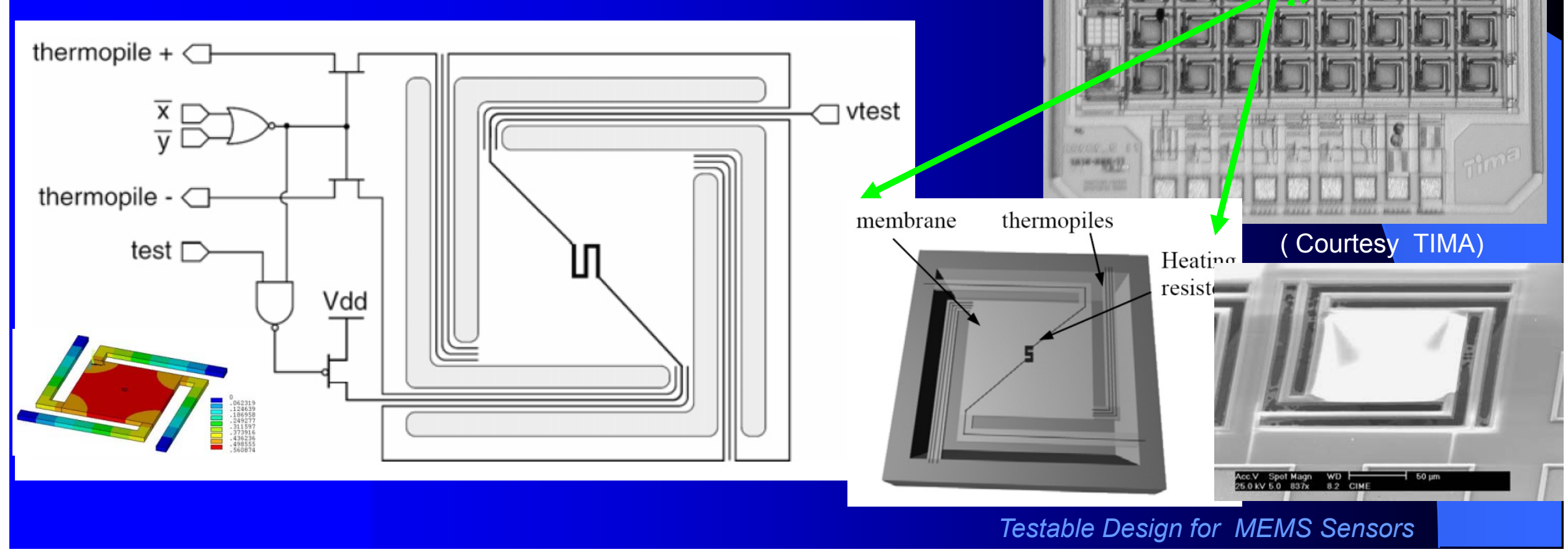




\section{Self-Test Principle IR Imager}

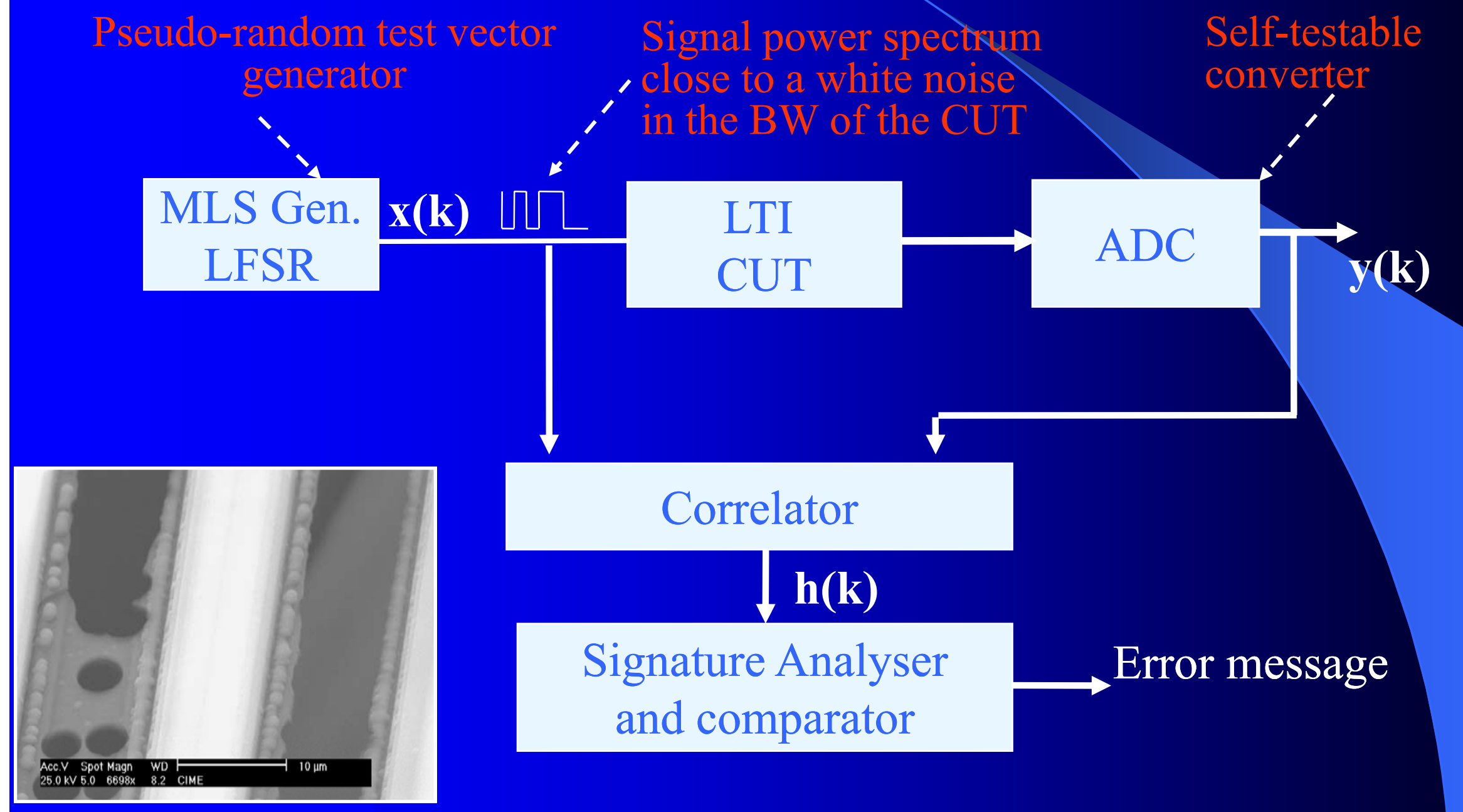




\section{Digital (Droplet) Microfluidics}
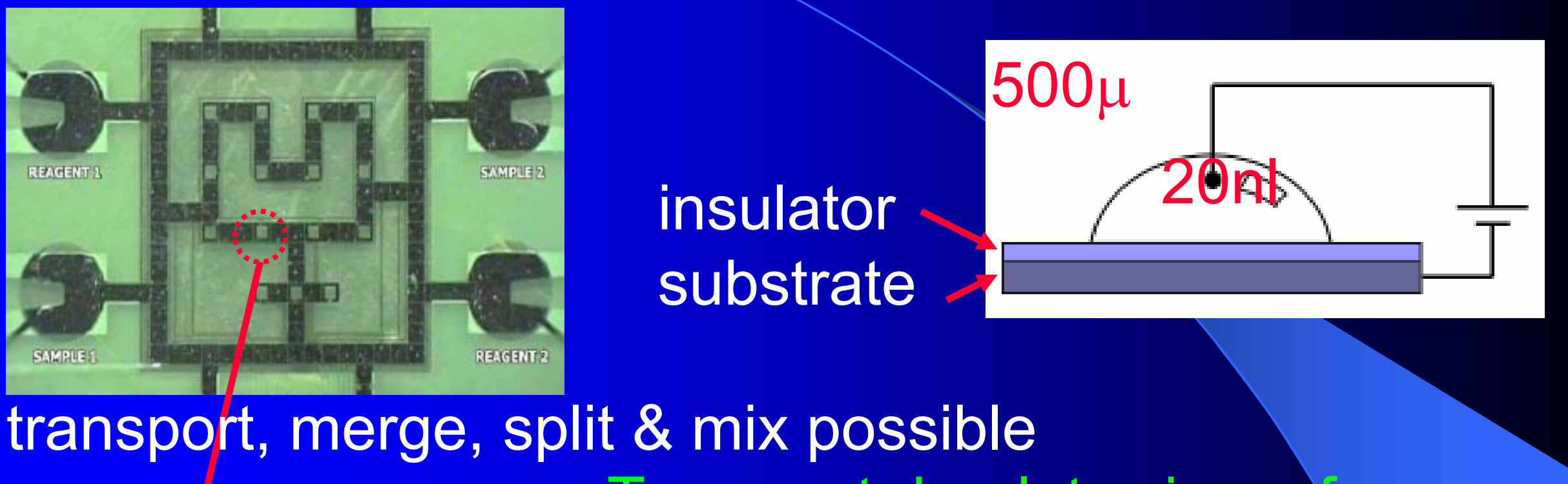

transport, merge, split \& mix possible

Transport droplets via surface

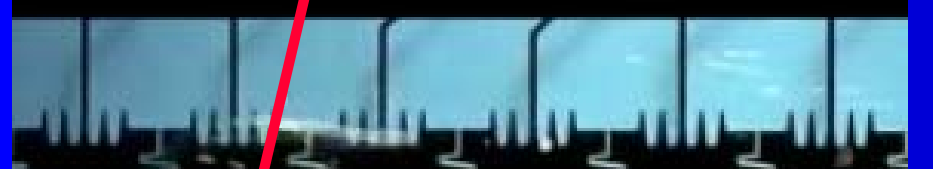
tension control. Resembles CCD

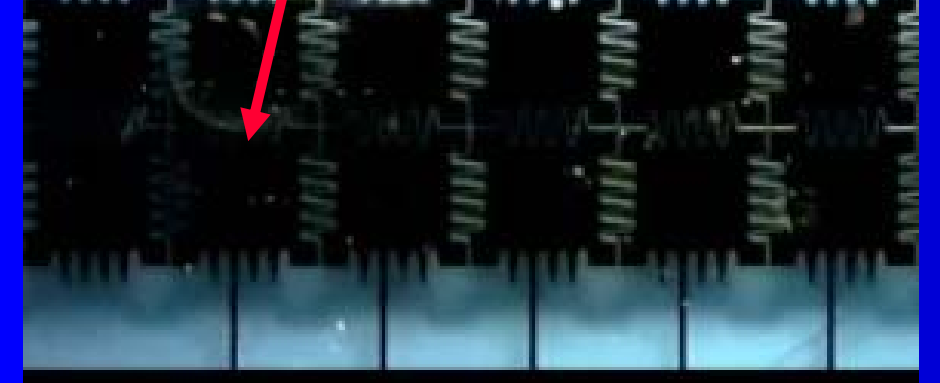
clocking mechanisms.

( Courtesy Duke Uni.)

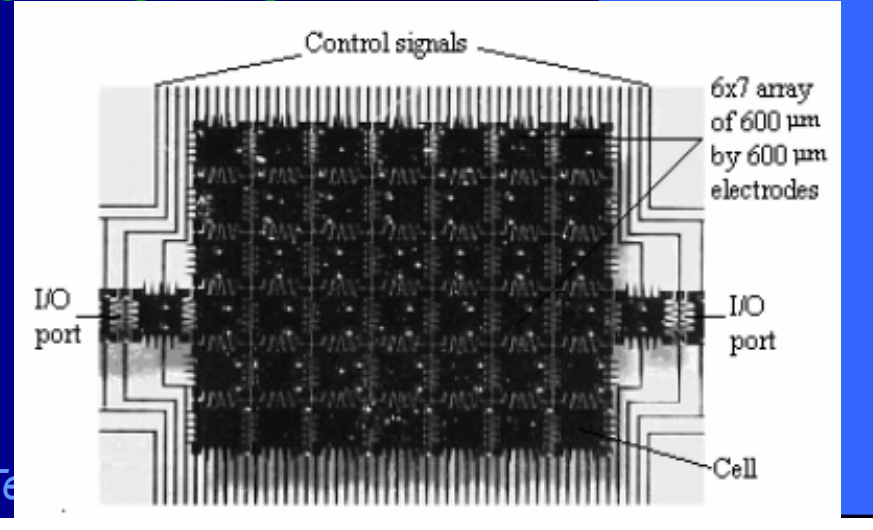




\section{Testing Digital (Droplet) Microfluidics}

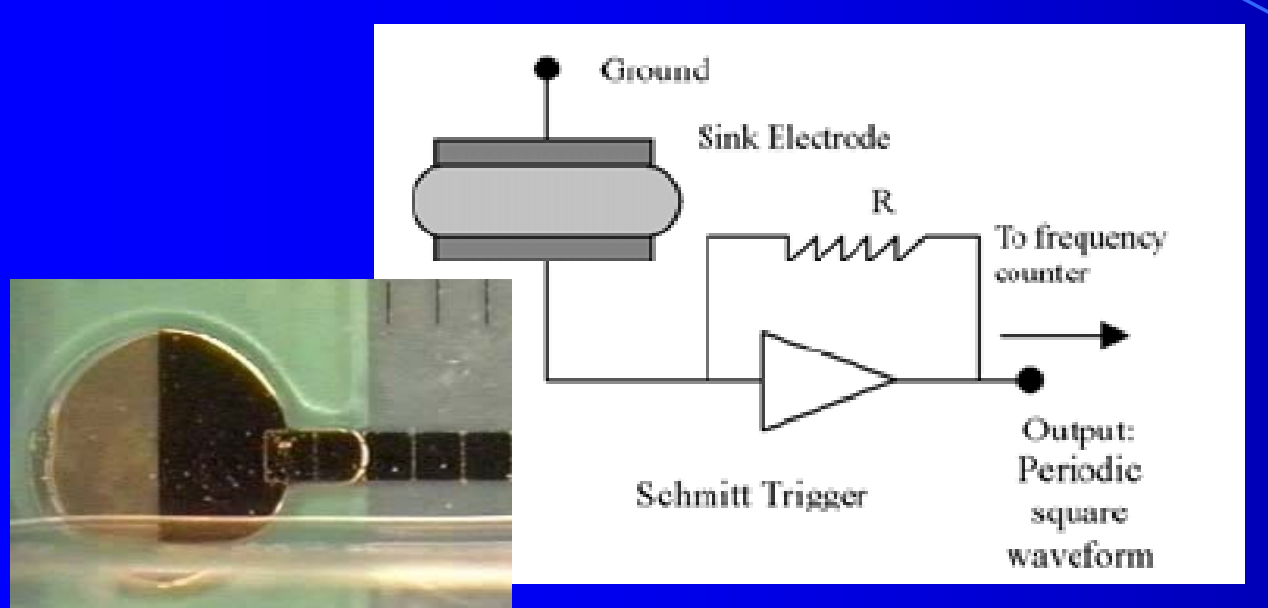

inject, detect \& concurrent testing

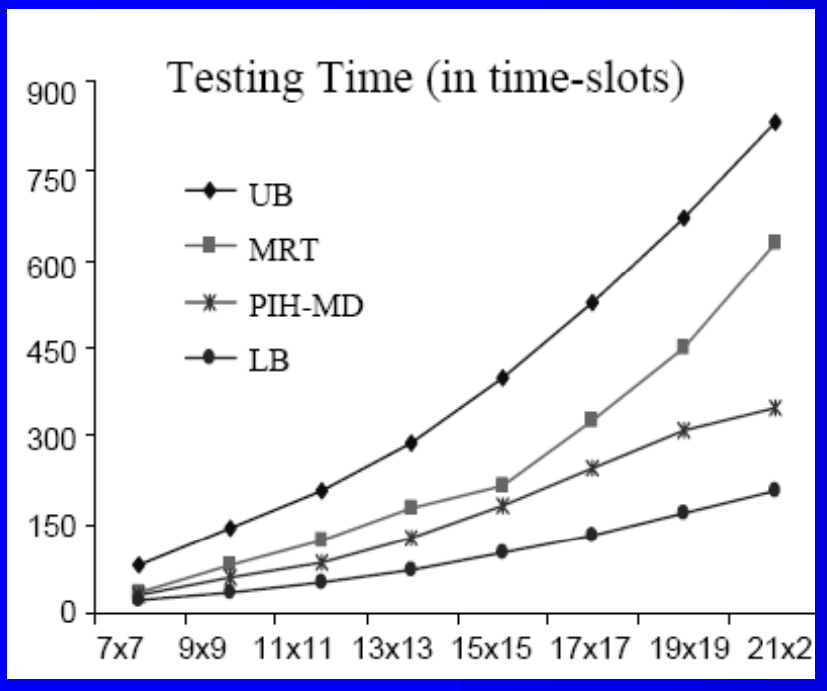

also BIST

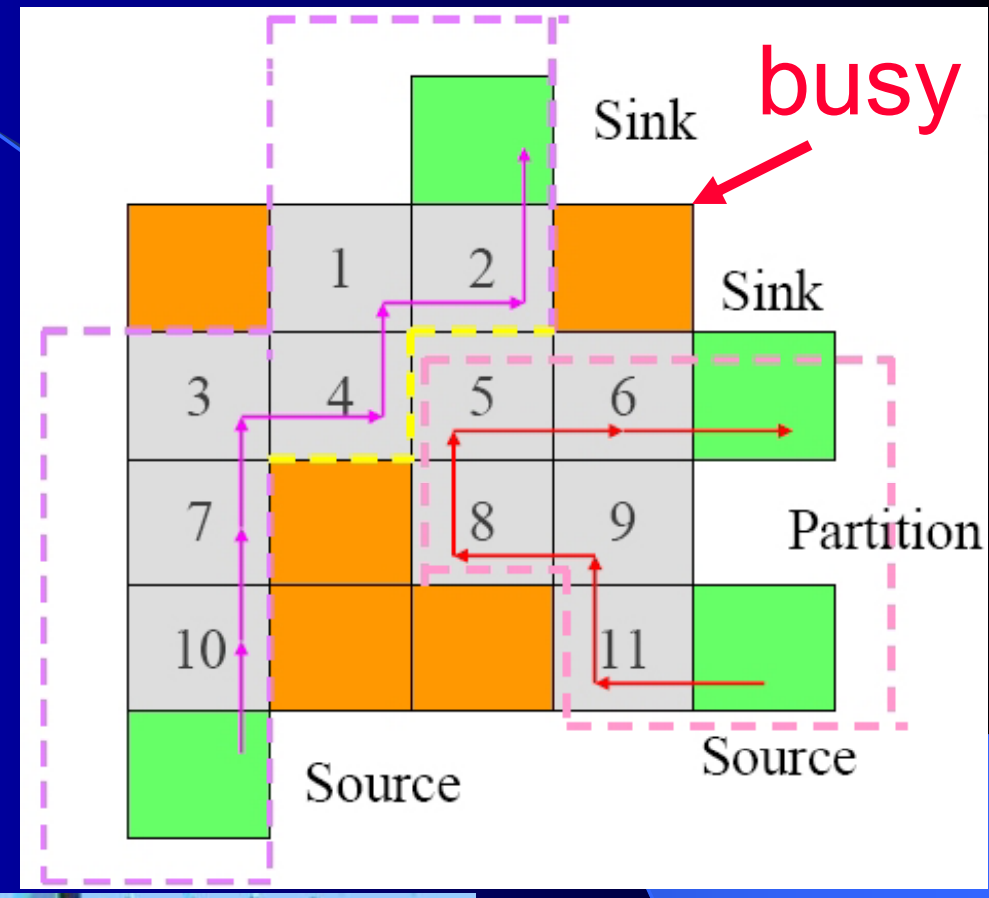

and reconfigeand reconig

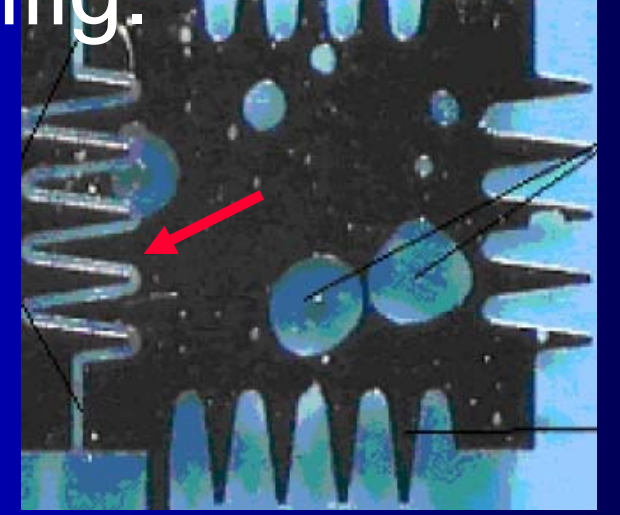

Isolation degraded electrode 


\section{Anchor, Beam and Finger Defects}

Several defects cannot be detected by specification based tests such as resonant frequency and sensitivity
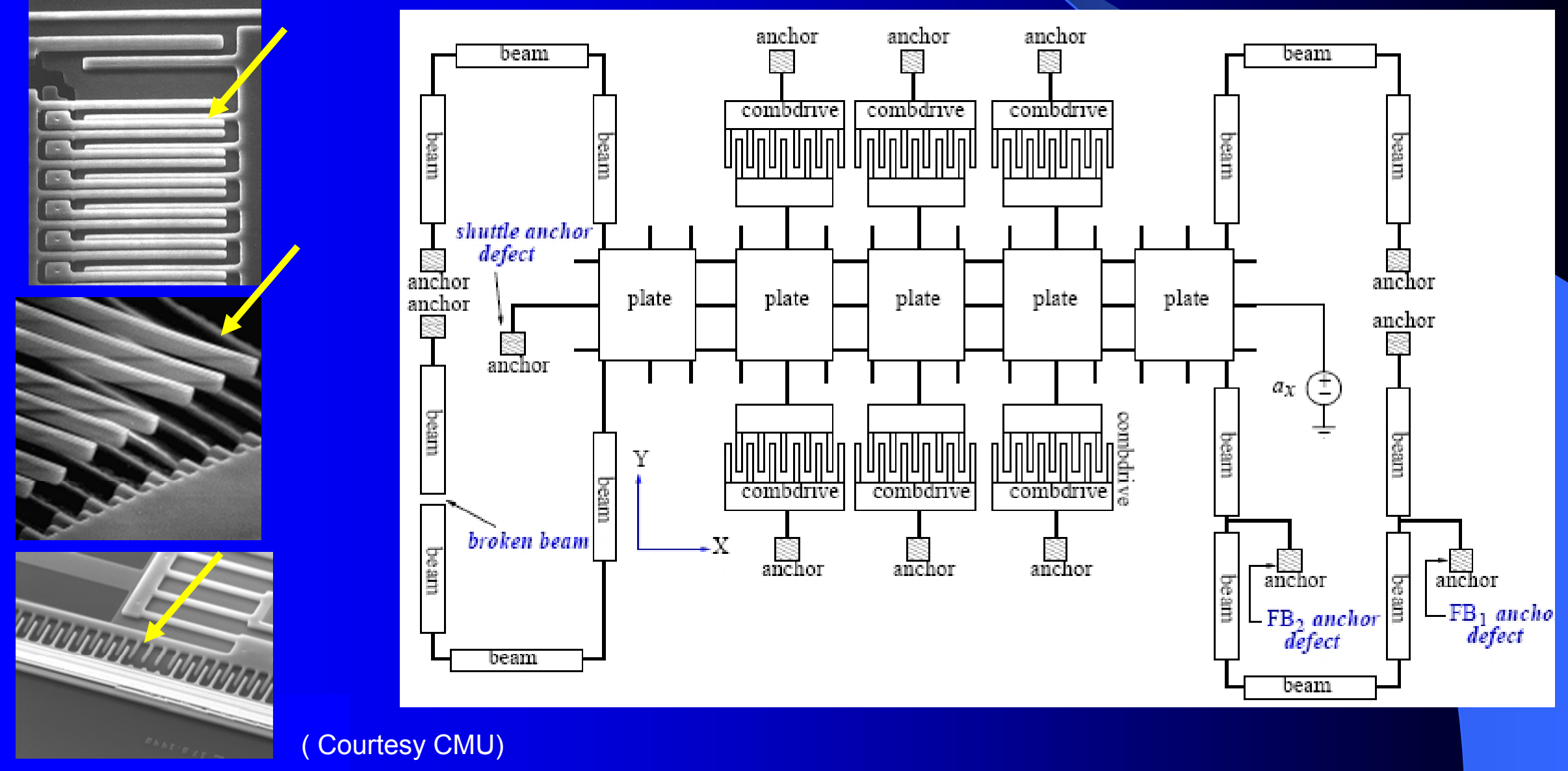

( Courtesy CMU) 


\section{Selftesting Accelerometer}

Two actuations are used and two nodes are compared. This results in wafer defect detction capability

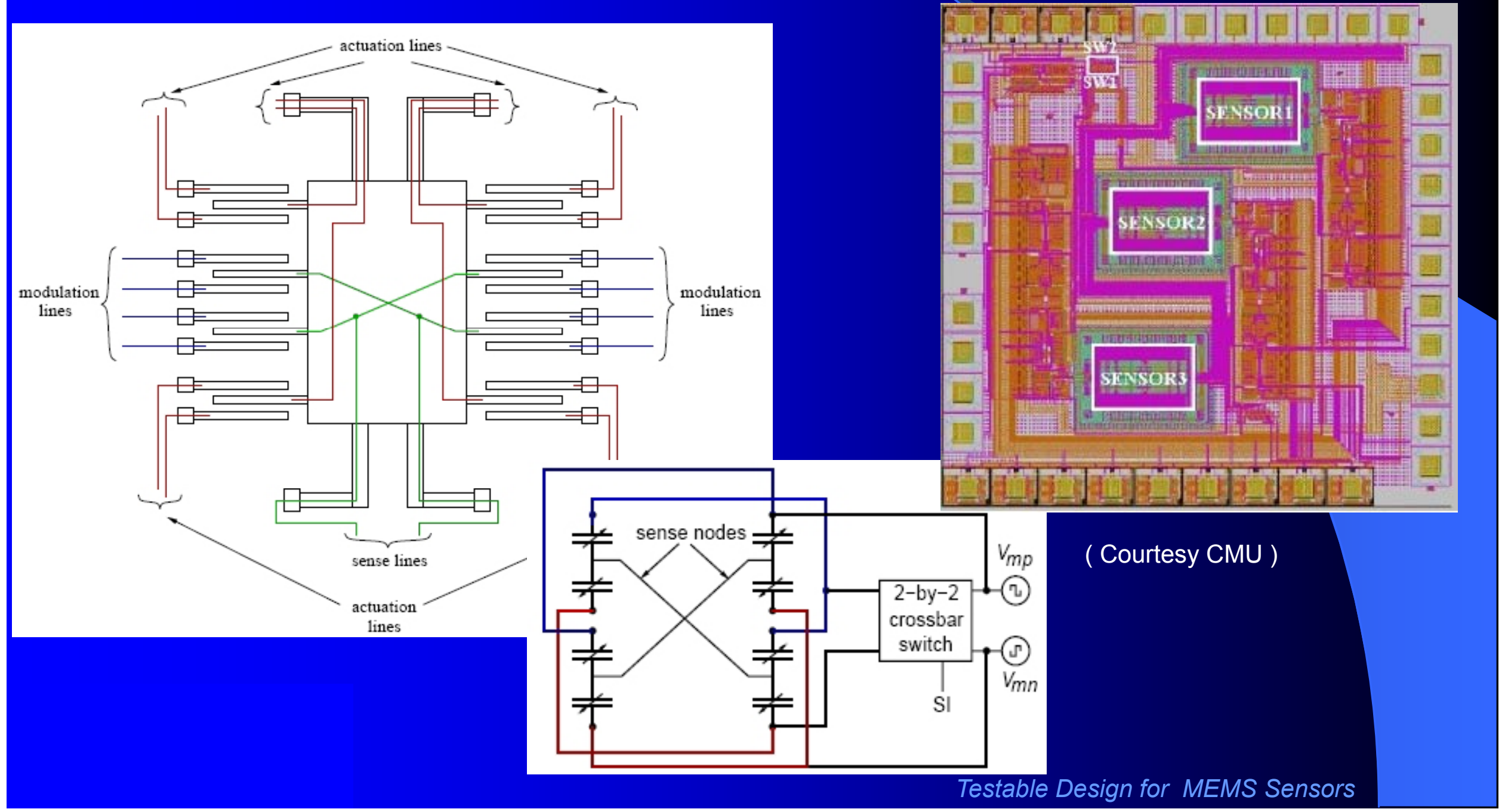




\section{Selftest Accelerometer using M-field}

magnet

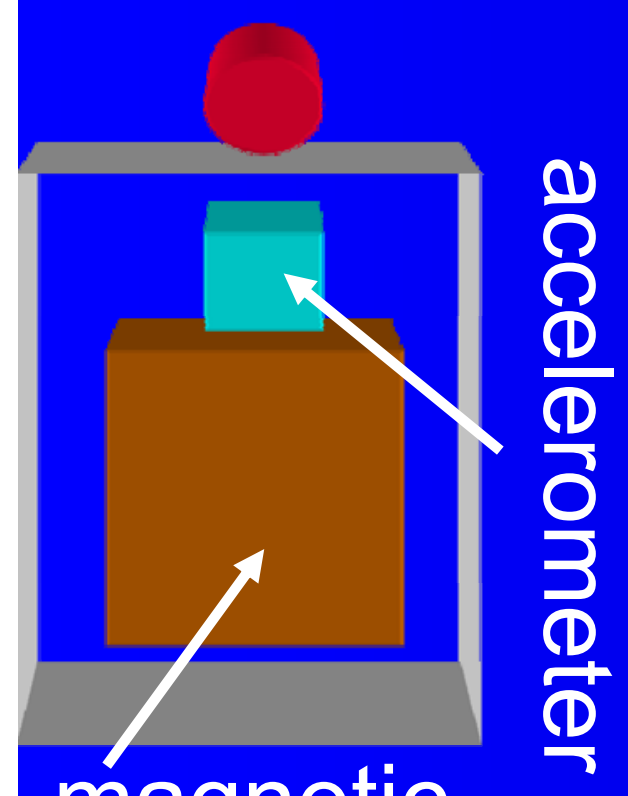

magnetic

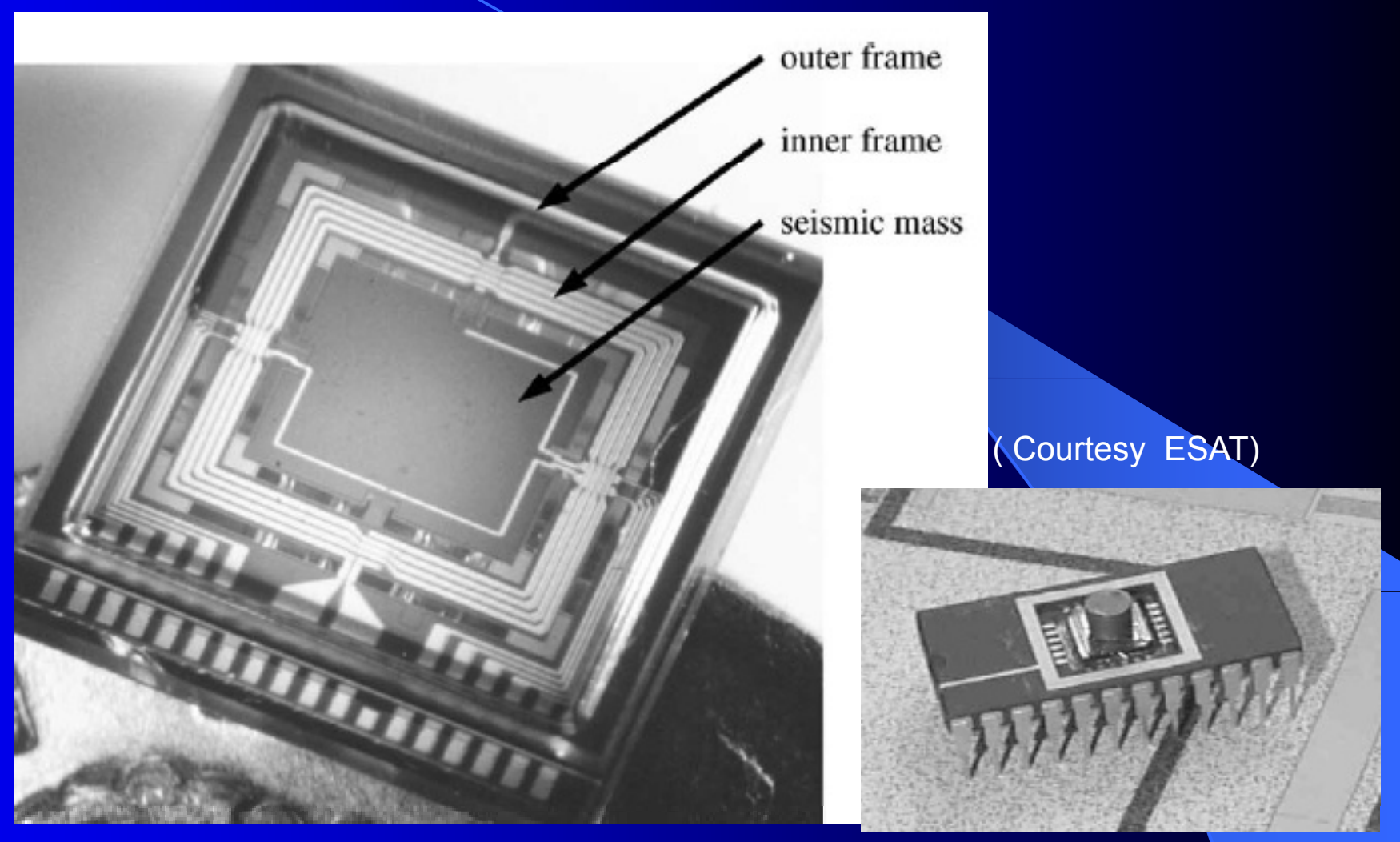

micro resonator

Uses permanent magnet and suspended beam Al coil.

Provides more (elec.st.) realistic movements (14 g) 


\section{Conclusions}

Testing of MEMS-based microsystems is one of the potential show stoppers for mass production

Specification-based testing done predominately, defect-based hardly just like in analogue microelectronics

- Large local effort in MEMS handlers

Relatively little knowledge on defects or how to detect them. System simulation required for quality!

Very little DfT or BIST on-board currently. Expected to come soon. Structural test is way to go. 


\section{Questions}

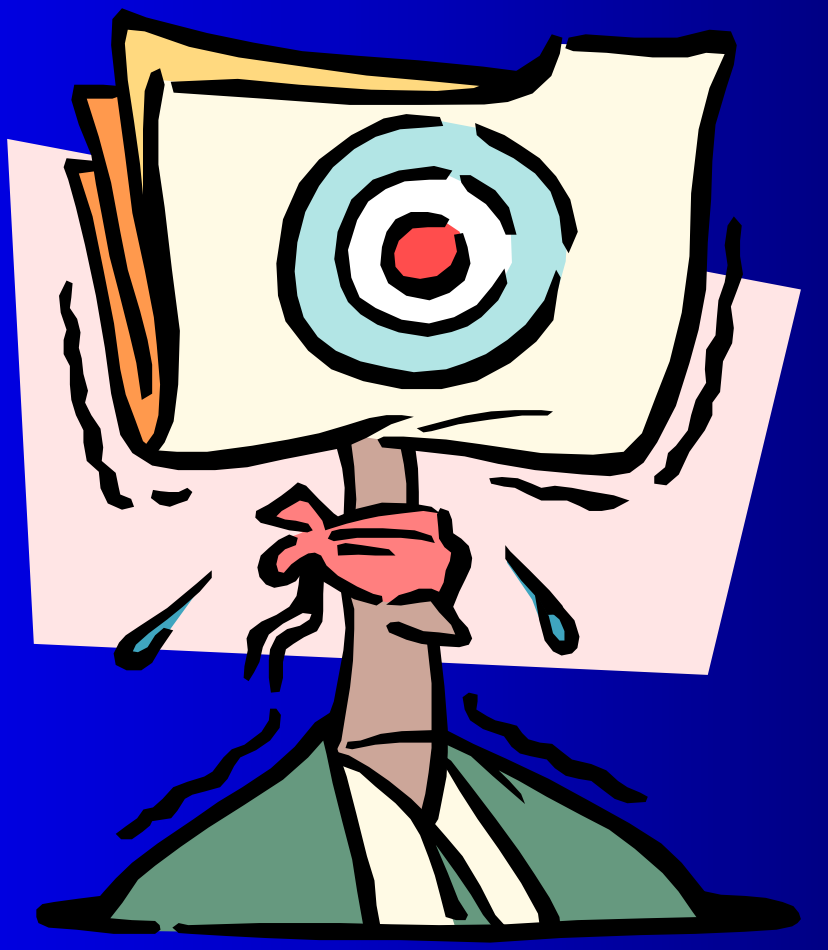

